\title{
CARE-ORIENTED PRACTICE IN AT-RISK UNITS: RISKS, REALITIES AND THE ROLE OF MULTI- DISCIPLINARY TEAMS
}

By

Alexis Harris

A thesis submitted in fulfilment of the requirements for the degree of Master of Arts in Criminology

School of Social and Cultural Studies

Victoria University of Wellington

July, 2015 
At-Risk Units (ARUs), which contain and prevent suicide and self-harm among prisoners, have been criticised for their isolating, non-therapeutic nature. This thesis explores the potential for care-oriented practice to develop in ARUs at two prisons, with a particular emphasis on the role that multi-disciplinary teams and an enhanced healthcare presence can play in achieving this goal. Adopting a qualitative framework, this research draws upon nineteen interviews with nursing, forensic and custodial ARU staff from Hawkes Bay Regional Prison (HBRP) and Rimutaka Prison (RP). This research found that while normative care-oriented operational safeguards and legal frameworks underpin current ARU policies, they can often become shaped, or in some cases inhibited, by managerial adherence to compliance, risk-management priorities, limited resourcing, staffing issues and a punitive prison culture. However, in instances where multi-disciplinary teams are well resourced, have open channels of communication and operate within health-focused ARU environments, as evidenced in the current workings of RP, positive care-oriented responses to 'at-risk' prisoners can be better provided. The thesis concludes by noting that incremental reforms to the current framework may be useful in enhancing care-oriented ARU practice. However, even with change, the question remains whether correctional ARUs can stem burgeoning mental health issues and 'at-risk' behaviours among prisoners. 


\section{Acknowledgments}

I would like to warmly acknowledge a number of people for their assistance, patience and encouragement during the course of my thesis. While writing my thesis was an incredibly rewarding and enriching experience, it was at times challenging and a process that was hugely aided by the support of some great people who I would like to briefly acknowledge.

This thesis would not have been possible without the assistance of the Department of Corrections, particularly the staff from Offender Health, Research \& Evaluation and the two correctional sites considered for this research. Thank you for allowing this project to happening and, in many cases, for taking an active interest in the wider goals of this project. In particular, I would like to thank the nineteen participants who agreed to take part in this research and who were hugely accommodating to me during my site visits. Your willingness to have open and forthright conversations was both personally stimulating and central to the completion of this thesis. I hope that this thesis accurately presents your views and experiences and brings some newfound appreciation to the important role each of you play within the ARU and the prison more broadly.

I would like to thank all the staff and students in School of Social and Cultural Studies, who have been a huge help with my studies and wider university experience over the past few years. In particular, I would like to thank Cara Gledhill, who has been a constant source of support, for a number of my academic projects and pursuits to date, and who provided me with useful guidance and feedback throughout the course of this thesis. Thanks to Charles Sedgwick for the engaging conversations and for stimulating my interest in this topic, in its earlier days. Thank you to Charis Dixson, for keeping me company in our office and for keeping me relatively sane over the last year, and to Samantha Keene for your constant advice and reassurance.

Thank you to my friends and family who have been there for me over the past year. My parents and my two brothers, in particular, have been constant sources of love and support throughout this period, and for many years prior, despite being scattered across the globe. Thank you to Jack, for engaging with my little rants and constant 
'thesis updates', for always keeping me entertained and for distracting me when I needed it most. Your love and encouragement has been so central to getting me to the finish line.

Lastly, and most importantly, I would like to thank my academic supervisor, Elizabeth Stanley. Your help, expertise, encouragement and useful discussions along every step of the way has been a tremendously positive influence to this research and to my postgraduate experience more broadly. I could not have asked for a better supervisor and mentor and I owe you greatly for the support you have lent to this project. 


\section{Abbreviations}

ARU - At-Risk Unit

CCHDB - Capital \& Coast District Health Board

CO - Corrections Officer

HBRP - Hawkes Bay Regional Prison

IOMS - Integrated Offender Management System

MDT - Multi-Disciplinary Team

MHST - Mental Health Screening Tool

PCO - Principal Corrections Officer

PSOM - Prison Service Operations Manual

REGC - Research and Evaluation Governance Committee

RP - Rimutaka Prison 


\section{Table of Contents}

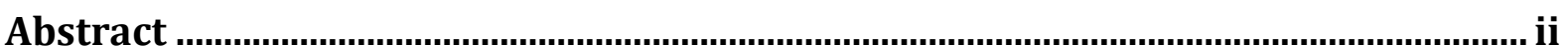

Acknowledgments.........................................................................................................

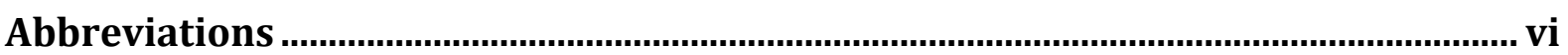

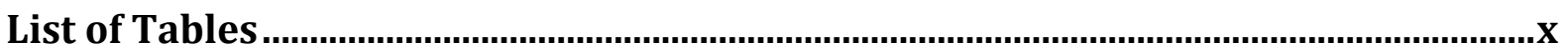

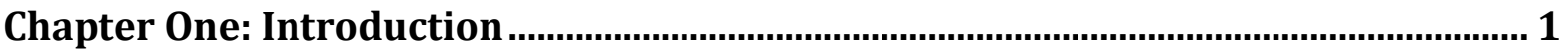

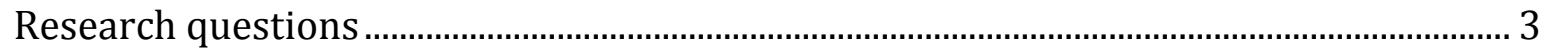

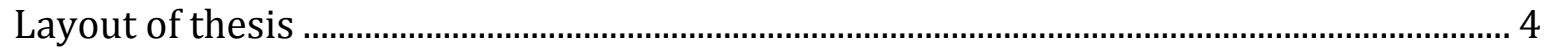

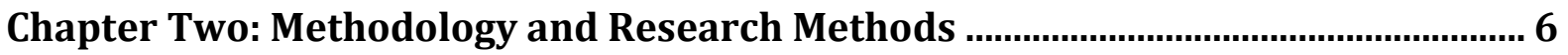

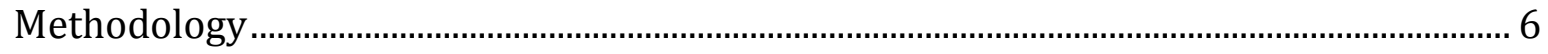

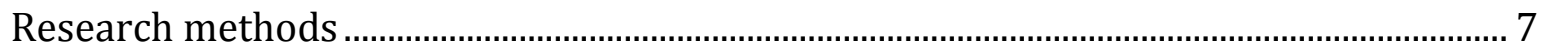

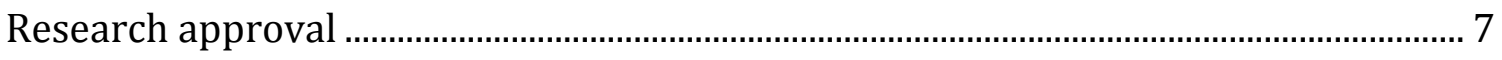

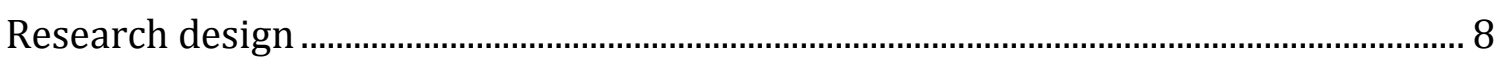

Changes from the initial proposal.....................................................................................

Research locations .............................................................. Error! Bookmark not defined.

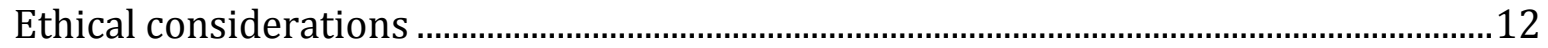

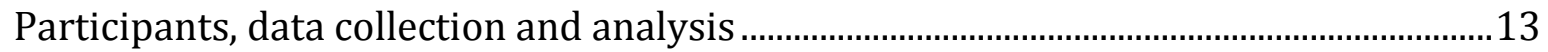

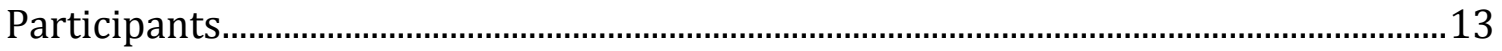

Recruitment and interview schedule .............................................................................. 15

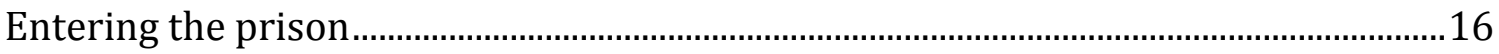

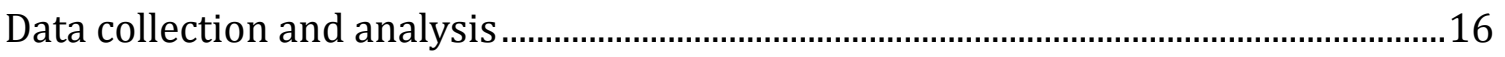

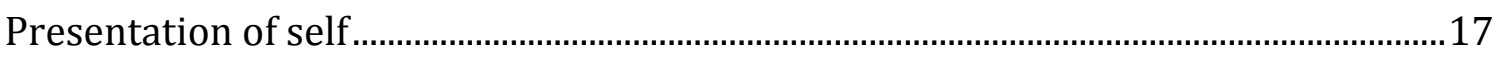

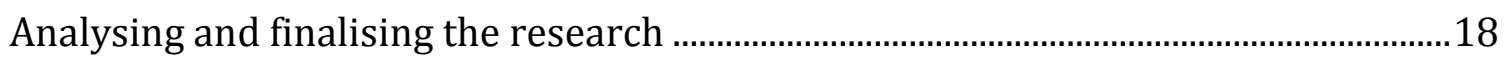

Chapter Three: Conceptualising a Care-Oriented Approach to 'At-Risk' Prisoners

20

Suicide and self-harm in prison as complex phenomena.....................................................20

Legal obligations relating to care-oriented responses to 'at-risk' prisoners ...................21

'Humane containment' and equivalence of care ...............................................................21

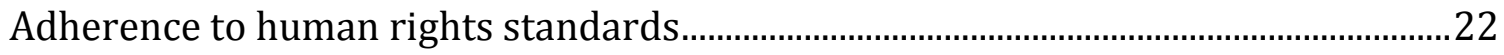

The current ARU system: criticisms and progressive policies............................................26

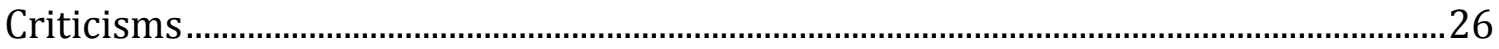

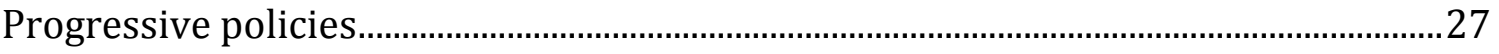

The role of staff and multi-disciplinary workings within ARUs ...........................................30

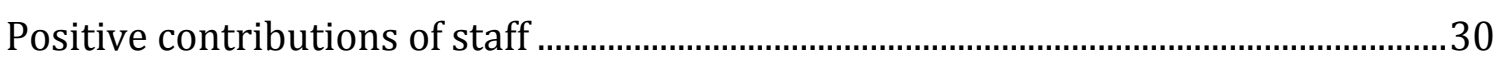


Training and resourcing 30

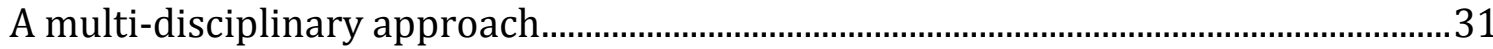

Multi-disciplinary workings: custody vs. care roles .............................................................. 32

Systemic and institutional barriers to care-oriented practice ..............................................33

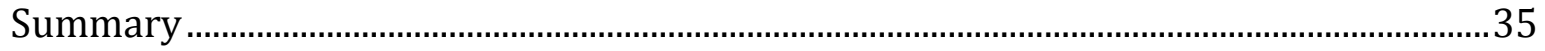

Chapter Four: The New Zealand 'At-Risk' Framework - Commentary and Practice

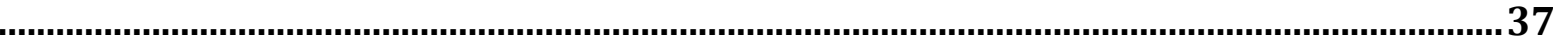

Mental health, suicide and self-harm rates in New Zealand prisons ....................................37

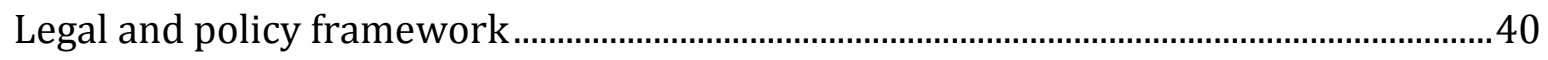

Strategic approach to management of self-harm and suicide risk .....................................43

What are At-Risk Units? ......................................................... Error! Bookmark not defined.

Day-to-day operations....................................................... Error! Bookmark not defined.

Commentary and issues raised in New Zealand ......................................................................4

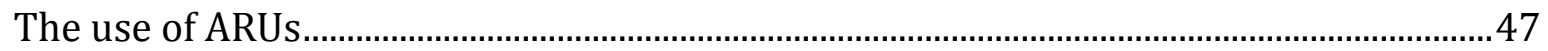

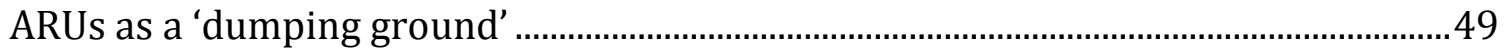

What gives rise to the overuse and misuse of ARUs? ......................................................... 51

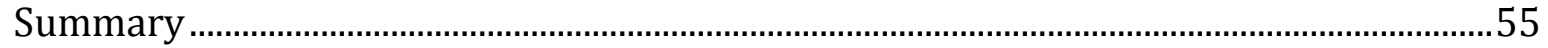

Chapter Five: 'Risk from Above' and Organisational Risk Management in ARUs...57

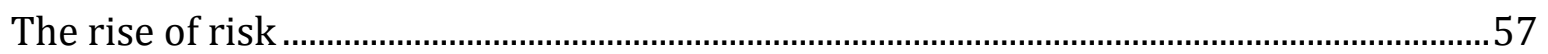

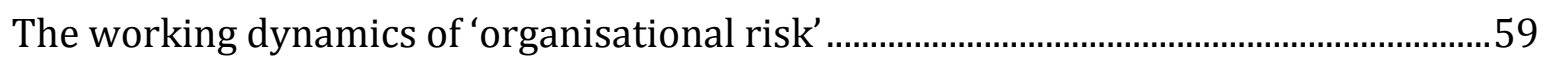

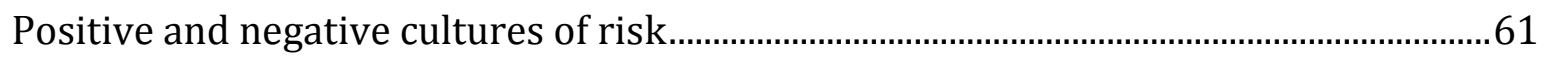

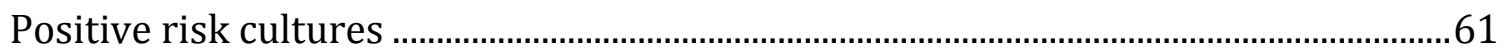

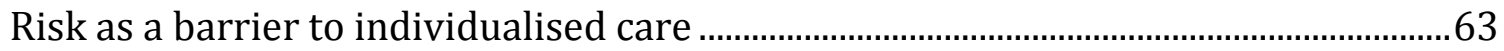

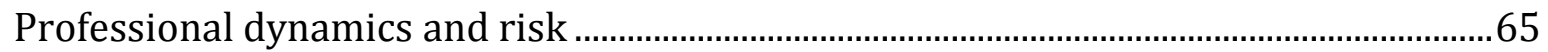

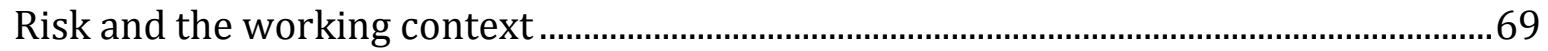

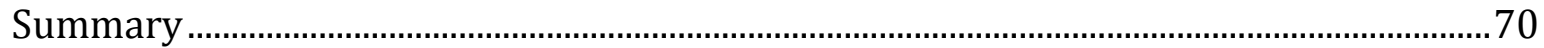

Chapter Six: Multi-Disciplinary Teams and Ground-up Pathways to Care ..............72

The working dynamics of custody and care ............................................................................. 73

Occupational constraints and organisational barriers to care ............................................77

RP's ARU: Towards a successful MDT model.......................................................................82

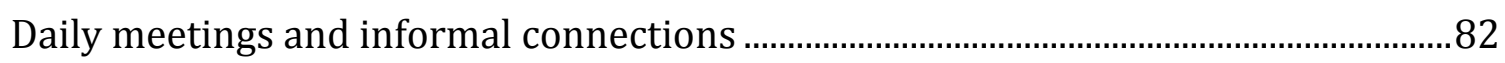

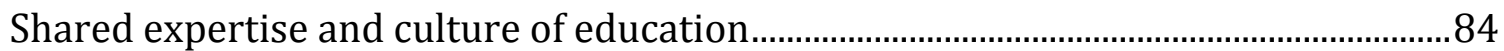

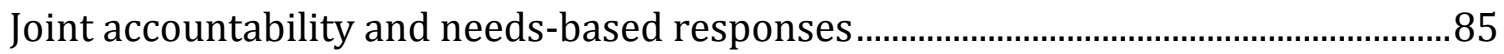

Integration with wider prison community and other positive practice .........................87

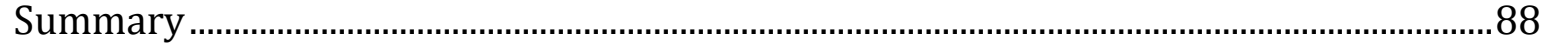

Chapter Seven: Conclusion - Thinking about Change ........................................................ 89

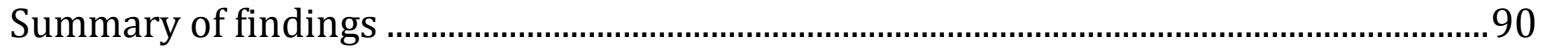




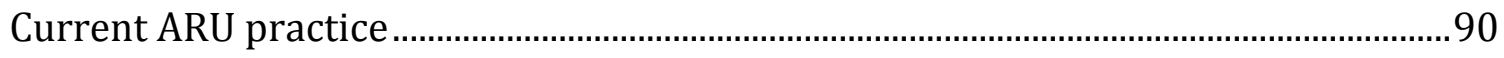

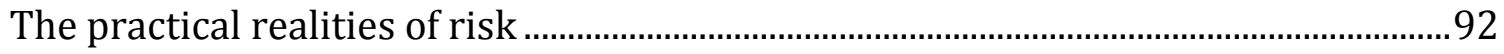

Positive risk cultures and MDT workings ............................................................................. 93

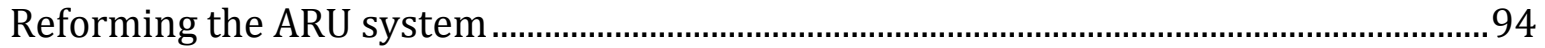

The centrality of cultural and operational factors............................................................... 94

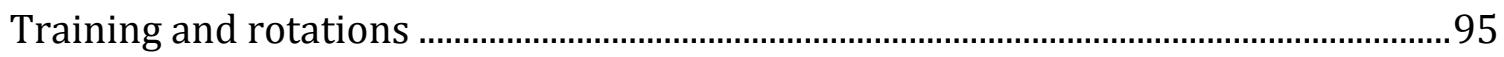

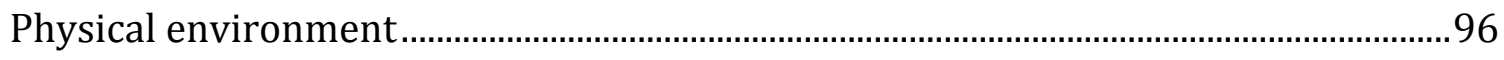

Prisoner vulnerability and the wider prison environment.............................................97

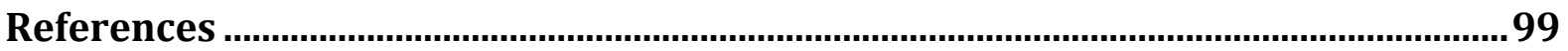

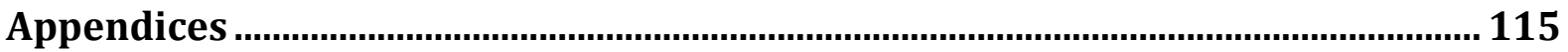




\section{List of Tables}

Table 1: Participant Demographics I

Table 2: Participant Demographics II

Table 3: Incidences of self-harm and suicide in New Zealand prisons, 2005-2015

Table 4: Number of prisoners and placements in RP and HBRPs' ARUs, 1 June - 31 August 2014

Table 5: Reasons for admission to ARU in RP and HBRP, 1 June - 31 August 2014 


\section{Chapter One: Introduction}

International research suggests that prisoners are three to five times more likely to have diagnosable mental health issues than the general population (Harvey et al., 2009). In New Zealand, the most recent major investigation highlighted that prisoners are over five times as likely to be affected by mental health illnesses and disorders as the general public (Simpson et al., 1999). In New Zealand, and around the world, rates of self-harm and suicide in prison are consistently higher than that of the general population (Liebling, 2006; WHO, 2007; Department of Corrections, 2014a).

While the provision of adequate suicide prevention strategies is central in reducing selfharm and suicide in prison (Gallagher \& Dobrin, 2005), such responses are restricted by the nature of prisons where security concerns and risk management may be prioritised over the needs of individuals (Wakem \& McGee, 2012). Similarly, while many states take steps to proactively protect prisoners from harming themselves via the development of sound policy, such policies may not always be administered or implemented in a careoriented manner. Accordingly, developing appropriate responses for self-harming and suicidal - or 'at-risk' - individuals within a prison environment is a challenging task, for both policy-makers and operational staff alike. The task of developing appropriate responses is made difficult by the fact that self-harm arises from complex motives and is not always indicative of a suicide attempt or suicidal ideation (Groves, 2004). Self-harm can act as a means of reclaiming a sense of personal autonomy, or to undermine or resist prison authorities (Groves, 2004). For this reason, this thesis frames suicide and self-harm somewhat simplistically, with suicide representing the most 'extreme end of a continuum of self-injurious behaviours', unless expressly perceived or discussed otherwise by staff interviewed (Burrows et al., 2003: 7).

A common way in which correctional suicide risk is managed is through the use of suicide-prevention cells within At Risk Units (ARUs). ARUs are dedicated, purpose-built facilities within the prison complex where prisoners who are considered 'at-risk' to themselves are placed, for assessment and to ensure their physical health is kept safe (Wakem \& McGee, 2012). ARUs are commonly used in New Zealand prisons, and they 
help to reduce suicide among 'at risk' prisoners (Department of Corrections, 2013a). Many governments ${ }^{1}$ have practically endorsed their use as they seem to provide a secure environment to monitor, assess and protect 'at-risk' prisoners from harming themselves.

ARUs have however been criticised for serving a punitive rather than therapeutic purpose (Roguski \& Chauvel, 2009). Critical commentators have described ARUs as a paradoxical solution to prisoners who are 'at-risk', noting they exhibit a 'fundamental contradiction between custody and care' (Scraton, 2007: 46). ARUs provide an environment for behaviour to be controlled, but their physically restrictive and isolating nature can exacerbate existing issues and contribute to longer-term mental health problems for prisoners (Coid et al., 2003; Anasseril, 2006; Hennessy, 2011). Some researchers argue that ARUs only address 'surface manifestations' of prisoner distress and cannot provide longer-term responses for recovery. They enable a 'death-avoidance approach' to prisoner care (Scott \& Codd, 2010: 104; Cliquennois \& Champetier, 2012: 397).

Despite these criticisms, ARUs do seem to advance elements of care that tend to be missing from the mainstream prison environment. One element, that forms the focus of this thesis, is that ARUs are increasingly staffed and aided by healthcare professionals working within the prison (Liebling, 2007). In New Zealand, Corrections Officers (COs), Department of Corrections-employed nurses, and contracted external forensic staff (nurses and psychiatrists) employed by the District Health Board (DHB) are all involved in the running of the ARU and the management of 'at-risk' prisoners. In many instances, the contribution of external health providers has led to positive results and produced an increasingly care-oriented environment (Barker et al., 2014). Yet, these efforts to enhance care-oriented responses to prisoners via the introduction of multi-disciplinary teams (MDTs) have been subject to limited attention.

Little academic consideration has been given to the way in which 'at risk' prisoners are responded to and how their management or care is practiced 'on the ground'. Further, we are yet to understand the relationships between external healthcare

\footnotetext{
${ }^{1}$ For example, 'Safe Observation Cells', or 'Muirhead Cells', in Victoria (VIC), Australia; 'Safe Cells' in New South Wales (NSW), Australia; and, 'Safer Cells' in England \& Wales.
} 
providers/agencies and the Department of Corrections, and how these relationships can contribute to different treatment approaches towards prisoners. Internationally, research has highlighted high levels of interdisciplinary conflict across MDTs, particularly in terms of conflicting managerial approaches when dealing with selfharming or mentally unwell prisoners (Ramluggun, 2013). In order to enhance a more care-oriented approach to prisoners in distress, it is therefore important to understand how staff members balance competing disciplinary or cultural priorities with prisoner care, on a day-to-day basis (Liebling et al., 2005). This thesis hopes to achieve an understanding of how staff grapple with such priorities to the extent that they progress or impact on care-oriented practice, within the complex working environment of the ARU.

\section{Research questions}

In light of the above considerations, this thesis will seek to identify and consider how multi-agency collaborations can contribute to the development of care-oriented practice and responses for male prisoners in New Zealand's ARUs. This will be explored via the following research questions:

1. How has the policy surrounding ARUs and their international equivalents developed in recent years? In particular, in what ways, if any, have more careoriented 'at-risk' policies been developed or implemented?

2. How do ARUs currently operate in New Zealand 2 ?

3. How is the policy relating to ARUs interpreted by the staff who work within them?

4. In what ways have multi-agency collaborations contributed to the development of care-oriented responses for male prisoners in New Zealand's ARUs?

5. Has any other good practice emerged from the introduction of MDTs in New Zealand's ARUs?

6. Are there any constraints on care-oriented responses to 'at-risk' prisoners within the current system?

7. Are there any unintended outcomes from the creation of multi-disciplinary ARUs in New Zealand?

\footnotetext{
2 Considering ARU practice in RP and HBRP.
} 
Within this, the research will have the following objectives:

a) To identify how the development of multi-disciplinary collaborations within two New Zealand ARUs is perceived and experienced by staff;

b) To analyse the way in which competing 'security' and 'care' priorities are balanced by staff working in certain New Zealand ARUs, on a day-to-day basis;

c) To explore the current impacts, limitations and bureaucratic constraints of a risk-averse culture in terms of providing care to prisoners;

d) To produce a set of key 'take-home' considerations for the Department of Corrections, to guide future policy and operational development in this area.

Overall, this thesis will contribute to our limited knowledge about ARU practice in New Zealand and the extent to which MDTs and an enhanced healthcare presence can encourage a care-oriented approach when responding to 'at-risk' prisoners.

\section{Layout of thesis}

This research explores the extent to which care-oriented practice can be enhanced in ARUs by exploring staff perceptions of practice across two correctional sites in New Zealand. It highlights the important role that MDTS can play in overcoming systemic or institutional barriers to care, such as a focus on organisational risk management, particularly when underpinned by cohesive, health-centric working cultures. The structure of this thesis has been largely guided by the research findings and subsequent data analysis, and consists of seven chapters.

The following Chapter Two sets out the methodological approach for this thesis and outlines the research methods used, including some limitations and personal challenges that arose during the course of the project. Chapter Three sets out the background and conceptual underpinnings of ARU policy and practice, including some international debates and developments in relation to the potential for care-oriented practice. It also presents some frequently cited institutional and systemic barriers to care and highlights the potentially important role that staff can play in addressing to prisoners' needs.

While commentary relating to ARU practice in New Zealand is relatively scarce, Chapter Four presents New Zealand's current ARU framework and system and highlights some 
of the criticisms that have been raised in respect to its current approach to prisoner care. Chapter Four introduces some initial research findings as a way of further contextualising the existing commentary, with a particular focus on the misuse and overuse of ARUs.

Risk, and the priority of risk management as a core institutional demand, was a key emergent theme that arose during the course of interviews, across both sites. Chapter Five explores the way in which risk is interpreted and manifested within the ARU setting, wielding both positive and negative implications for care-oriented approaches toward 'at-risk' prisoners depending on the organisational and cultural circumstances of the Unit. The chapter challenges some of the dominant discourses in the existing literature, which frame risk as an exclusionary or negative factor in practice, and considers the way in which risk is viewed differently by distinct disciplinary lenses.

Subsequently, Chapter Six explores the working dynamics of MDTs, with a particular focus on how 'custody' and 'care' priorities are balanced and perceived by staff from a range of disciplinary backgrounds. The chapter unpacks and presents the working dynamics of RP's MDT, framing it as particularly effective in delivering care-oriented approaches to prisoner care, for a number of reasons. Lastly, Chapter Seven concludes the thesis, providing some 'take-home' points for the Department of Corrections to consider, should they wish to progress a more care-oriented approach within the 'atrisk' context. 


\section{Chapter Two: Methodology and Research Methods}

\section{Methodology}

Within the international literature concerning ARUs, health care and prison work, a number of conceptual notions are continually raised. These include: risk management (O'Malley, 2010), staff well-being (Crawley, 2004), prisoner well-being (Liebling, 2006), 'humane containment' (King \& Morgan, 1980), managerialism (Feeley \& Simon, 1992), institutional power dynamics (Sim, 1990), the roles of fairness, trust or morality within the complex environment of a prison (Liebling, 2004) and environmental factors, in relation to suicide risk (Liebling, 2006). These 'themes' form a foundation to this analysis of ARUs in New Zealand, as the thesis explores how such themes converge and operate together to promote or inhibit care-oriented practice. The thesis is focused on improving the experiences of those currently in prison, by way of exploring the extent to which expertise, resources, collaborative partnerships and other ground-up developments can lead to positive staff practices.

The study follows an interpretivist methodology. This approach 'seeks to understand and appreciate the social world from the point of view of the individual' (Jupp, 2001: 12). Interpretivism seeks to unpack and explore 'an image of reality based on their own preferences and prejudices and their interactions with others', providing a useful lens to present staff perceptions within an area of the prison which has yet to be critically examined (Rafael \& Schutt, 2012: 49). The findings from this work are exploratory in nature and context-dependent, comprised of meanings or stories that correctional employees construct within their 'natural' setting (Gubrium \& Holstein, 2000). Applying an interpretive methodology allows for experiences and perceptions to be shared through the lens of 'prior experience, knowledge and expectations' (Rafael \& Schutt, 2012: 16). It is therefore a particularly useful framework to utilise for this thesis, which considers ARU practice from the perceptions of distinct disciplinary backgrounds and cultures of nursing, forensic and custodial working groups.

In the context of penal research, staff accounts have typically been overlooked and their role and wider influence in prison life has often been negatively stereotyped (Sharkey, 
2010). Further, prison staff have referred to themselves as an 'unvalued, unappreciated occupational group' (Crawley \& Crawley, 2008: 134). However, the dynamics of prison life are heavily intertwined and underpinned by 'complex and embedded relationships' and the decisions made, and the discretion afforded, by staff (Liebling et al., 1999). Accordingly, staff perceptions and experiences are well placed to convey the true working dynamics of ARU practice, in line with an interpretivist approach. Moreover, there is an emerging consensus that prison officers, and other staff, play a particularly important role in relation to positively identifying and responding to the needs of 'atrisk' prisoners, particularly given the time, proximity and relative intimacy shared between staff and prisoners (Rowan, 1994; Crawley, 2004; Sharkey, 2010). The vital role that staff play, and the obvious link they provide between prison policy and practice, makes them an 'essential group' when exploring the extent to which careoriented responses can be administered within the ARU environment (Short et al., 2009: 410). Bearing these sentiments in mind, staff were considered to be the most suitable and valuable group to interview for the purposes of this research. While prisoners would also be highly suitable and valued for this type of research, it was determined that staff would be better placed to discuss working dynamics and the drivers of ARU practice. Staff were also more accessible and, in early conversations, representatives from National Office seemed more open to the prospect of interviews with Corrections employees, rather than prisoners.

\section{Research methods}

\section{Research approval}

Correctional facilities worldwide have a reputation for being 'structurally and bureaucratically closed off from research' (Reiter, 2014: 417). Gaining entry has been described as the greatest challenge facing qualitative prison researchers, especially given the existing preference for quantitative research methods among state departments (Patenaude, 2001; Wacquant, 2002). Aligning my proposal with the Department of Correction's research strategy was also a crucial step in gaining access. I understood that a rigorous vetting process excluded research that was too politically sensitive or had limited practical value or tangible benefits to the Department. I was 
also aware that the resource-intensive nature of my proposed research, and my lack of experience 'in the field', could be barriers.

My initial proposal sought to unpack how ARU policy changes were perceived by staff, with particular reference to how this impacted on professional relationships within an MDT environment. My proposed data collection method comprised participant observation within the ARU and interviews with staff. I was advised by a Principal Research Advisor that this proposal would likely be declined by the Research and Evaluation Governance Committee (REGC) for several reasons: it placed too great a demand on staff and resources; the method of participation observation would be too risky in nature; and, importantly, the project outcome did not appear to be of high value to the Department.

Following this, I met with the Director of Offender Health, and corresponded with the Research and Evaluation Team, to reconfigure the proposal in line with the Department's research aims. Such 'collaborations' have been increasingly recognised as a useful, and often mutually beneficial, way of mitigating barriers to accessing prison systems for research (Reiter, 2014). A revised proposal was subsequently approved by the REGC. Following final acceptance, a formal Research Agreement was signed between myself and the Department, which outlined my responsibilities and expectations as a researcher (Appendix A). Upon signing the agreement, the Department was given full right of review of the thesis, in respect of any factual inaccuracies and any 'other content changes that it considers appropriate'. A Ministry of Justice criminal record clearance and proof of Human Ethics Approval from the University were also provided to the Department before commencing research.

Gaining institutional access was a lengthy process, taking nearly six months to finalise. However, I have no doubt that my intention to conduct interviews with staff 'at the coalface' added a level of depth and understanding about ground-level correctional practice that would have not been discerned through any other method.

\section{Research design}

My final research design comprises qualitative methods, focused on understanding working life from the perspectives of participants within their social context (Giacomini, 
2001). A qualitative approach usefully generates new insights into practices, experiences and social settings. For this thesis, semi-structured interviews were adopted in order to gauge understanding of current correctional practice in ARUs and to generate insight into the experiences and perceptions of ARU staff. Semi-structured interviews also allowed staff to share their stories and experiences in a flexible way. Alongside these interviews, this thesis draws upon data gathered from the Department's Integrated Offender Management System (IOMS) relating to 'at-risk' prisoners over a three month period, between 1 June and 31 August 2014. Other primary data, such as statistics relating to suicide and self-harm and official policies relating to 'at-risk' procedure, were also used. This official data provides valuable and important information to contextualise interview findings (Sapsford \& Jupp, 2006).

\section{Research locations}

Following consultation with National Office, it was decided that I should conduct fieldwork in two correctional sites, as a way of highlighting divergences in practice. It was mutually agreed the most ideal locations for interviews would be Rimutaka Prison (RP) and Hawkes Bay Regional Prison (HBRP), for a number of reasons. First, the sites are both male-only prisons ${ }^{3}$. I wanted to focus upon male 'at-risk' prisoners as most prisoners are male and they are at a higher risk of suicide than female prisoners (Department of Corrections, 2012a). Further, the treatment of mental health and emotional distress is highly gendered in nature, with male prisoners harbouring greater stigma in relation to mental health and emotional distress while incarcerated (Skogstad et al., 2006). International research also indicates that the experience of ARUs is an inherently different experience for female prisoners (Scraton \& Moore, 2005). As such, while researching female prisons is necessary to understand the wider operation of ARUs in New Zealand, it needs to be subject to separate in-depth attention.

Second, these sites were chosen as National Office staff thought that substantial variation in practice occurred between the ARUs of RP and HBRP. They expressed interest in exploring why such operational divergences occurred - for example, did it relate to the different environment, a different culture of working or differences in understanding policy, or some other reason? While the findings of this thesis paint, at

\footnotetext{
${ }^{3}$ With the exception of transgender prisoners, who are housed in these prisons when identifying as male.
} 
times, some fairly stark contrasts between ARU practice in RP and HBRP, it is important to note that this thesis is not a comparative study. Third, and finally, RP and HBRP were picked due to their relative physical proximity to Wellington, which made practical access easier.

\section{Changes from the initial proposal}

The methods for this thesis have changed from the original proposal accepted by the REGC$^{4}$. These alterations are worth highlighting, in order to point out some of the 'false paths' I experienced over the research course - some of these challenges seem to be an almost inherent part of correctional research (Pautenaude, 2004; Liebling, 2014; Reiter, 2014).

On the advice of the Department, I initially proposed a mixed-methods research design. This approach included devising an international 'best practice' model of at-risk policy, via analysis of relevant overseas policy documents and relevant commentary. This exercise could be valuable in establishing what international best practice is, as well assessing how New Zealand policy compares to practice. I submitted a finalised review of international 'best practice' to the Department in late 2014. Generally speaking, New Zealand's approach was in line with the policies that I reviewed 5 . However, while official international policy documents were relatively easy to access, what ARU 'best practice' constituted was difficult to decipher given the scarcity of relevant commentary. Further, most academic analysis of analogous units is critical and unsupportive of ARUs, with little suggestion for reform or improvement, running counter to the development of a 'best practice' model (Scraton \& Moore, 2005; Coid et al., 2003).

The review also highlighted some limitations inherent in drawing 'best practice' from policy and operational sources only, a point also supported by my research findings. Such limitations include the ongoing divergences from, and inconsistent interpretations of, policy among many prison staff in their working practices (Smith, 2007; Carlton \& Segrave, 2014), which may be attributed to inadequate staff training or resource constraints (Gender \& Player, 2013). In line with international findings, many positive

\footnotetext{
${ }^{4}$ An 8-month progress report, outlining the changed approach to my research methods, was sent to the Department in April 2015. The Department approved the changes in May 2015.

${ }^{5}$ In line with a Departmental request, the review focused on New South Wales; Victoria; England \& Wales.
} 
working practices outlined by staff during interviews were organically developed and seldom mentioned in any official policy (Cox, 1984; Smith, 2007). It became clear that prison culture - the relationships or values shared by staff - also had a major role in shaping policy interpretation and subsequent practice (Cox, 1984; Liebling, 2004).

These factors made comparing 'best practice' of international/local policy somewhat of a redundant task. The ground-level experiences of prison life, which can be well deduced from staff commentary and observation during interviews (Liebling, 2004), provided a much sturdier platform to explore how ARU practice could be enhanced. As such, while no longer directly underpinning this research, this thesis draws on international policy developments where useful or relevant.

The Department also suggested that I review a 3-month 'snapshot' of 'at-risk' prisoners' management plans, to provide a contextual platform for interviews. However, major logistical barriers made this impossible. I was tasked with retrieving the plans myself, under the supervision of a Health Centre Manager temporarily based at the Department's National Office. To access plans, I had to first identify which prisoners were housed in the specified ARU sites during a designated three-month period. This was not a simple exercise, especially as this information was only available in hard-copy form within the prisons. In the end, in one prison, the names of prisoners who had been held in the ARU were read off a list down the phone. This was then checked against a spreadsheet, and again on IOMS for validity.

In the end, over 150 prisoners were identified as being held in the ARUs across the two sites in the three-month period, a much greater figure than originally expected. I then worked full-time alongside the Health Centre Manager for several weeks trying to retrieve their ARU management plans with little success, despite enormous perseverance on both our parts. Access was almost impossible in the research timeframe due to the fact that prisoner files travel with them, complicated further by the transient nature of ARU placement. The majority of the management plans had been dispersed across multiple locations, following the transfer or release of respective prisoners. Further, the hard-copy nature of the filing practices within the prisons meant that offsite access was logistically difficult. Copying, scanning and redacting the files was resource intensive and on-the-floor prison staff just did not have the time to assist this 
process. These difficulties illustrated how the 'extremely limited technological resources' in prisons can act as a major barrier to those attempting to secure groundlevel or operational data (Reiter, 2014: 421).

With the management plan exercise disbanded, I looked for a substitute. With agreement from the Director of Offender Health, I organised to access background material on the identified 'at-risk' prisoners from IOMS, again under the oversight of the Health Centre Manager. This ARU prisoner data comprises notes and other IOMS material relating to all prisoners housed across both correctional sites between JuneAugust 2014. It provides a useful 'snapshot' of quantitative data (e.g. number of prisoners, age, ethnicity), as well as some useful contextual information around prisoners' day-to-day experiences. Such primary data was useful in developing a more informed interview schedule and has also been used to support interview findings where relevant.

\section{Ethical considerations}

The research was accepted by the Victoria University of Wellington Human Ethics Committee (Appendix B). Full support was given by the Research Committee and no amendments to my original application were required. Research ethics generally take into account matters of informed consent, confidentiality, harm to participants and researcher integrity (Israel \& Hay, 2012). All these elements were considered when formulating my research.

Informed consent has been recognised as a 'fundamental guiding principle' in the context of ethical social research (Noaks \& Wincup, 2004). Participants were given an information form outlining the nature and purpose of the research, which was talked through prior to each interview (Appendix C), and they were given the opportunity to ask any questions about the research. Participants also signed consent forms (Appendix D) and recognised their participation was voluntary (Noaks \& Wincup, 2004).

Confidentiality and anonymity are central considerations in maintaining an ethically sound approach to research (Noaks \& Wincup, 2004). Participants' identities were known only to the researcher and all real names and other personal attributes have been omitted. All staff titles have been framed generically; for instance, 'Health Centre 
Managers' have been cited as 'Nurses' and 'Principal Corrections Officers' have been cited as 'Corrections Officers', for the purposes of enhanced confidentiality. All data relating to the project will be destroyed shortly after the thesis is completed.

Conducting interviews within a prison environment can raise unique ethical considerations. Attempting to establish trust and rapport in a difficult environment and mitigating power imbalances between researcher and participant are just two of the many issues highlighted by researchers in this field (Sim, 2003; Pautenaude, 2004; Reiter, 2014). I believe that these distinct ethical considerations were addressed by interviewing prison staff rather than prisoners, despite trust issues still existing with academics interviewing workers (Liebling et al., 1999). For instance, prison staff may be wary that their views may be fed directly back to National Office or managerial staff and be hesitant to speak critically about the working environment. While sensitive topics such as self-harm and suicide were raised in some interviews, staff understood that they did not have to answer questions that they did not feel comfortable doing so. In general, staff appeared open to my research, perhaps helped by my stressed independence from the Department as a researcher. In fact, many staff welcomed the opportunity to share their views about the ARUs and expressed hope that the Department would listen to their concerns and ideas.

\section{Participants, data collection and analysis}

\section{Participants}

In consultation with the REGC, it was proposed that the research would consist of 10-15 interviews. In the end, due to staff interest and availability, 19 interviews were conducted with nursing, forensic and custodial staff, to allow for a spread of perspectives across the disciplines. All participants were current employees of either the Department or Capital \& Coast District Health Board (CCDHB) and all currently worked in RP or HBRP's ARU, either directly or by way of external contract. The participants comprised seven corrections officers (COs), seven Department-employed nursing staff and four externally contracted CCDHB forensic staff (see Table 16).

\section{Table 1: Participant Demographics I ( $\mathrm{N}=18)$}

\footnotetext{
${ }^{6}$ The data in this table has been removed, after a request from the Department.
} 


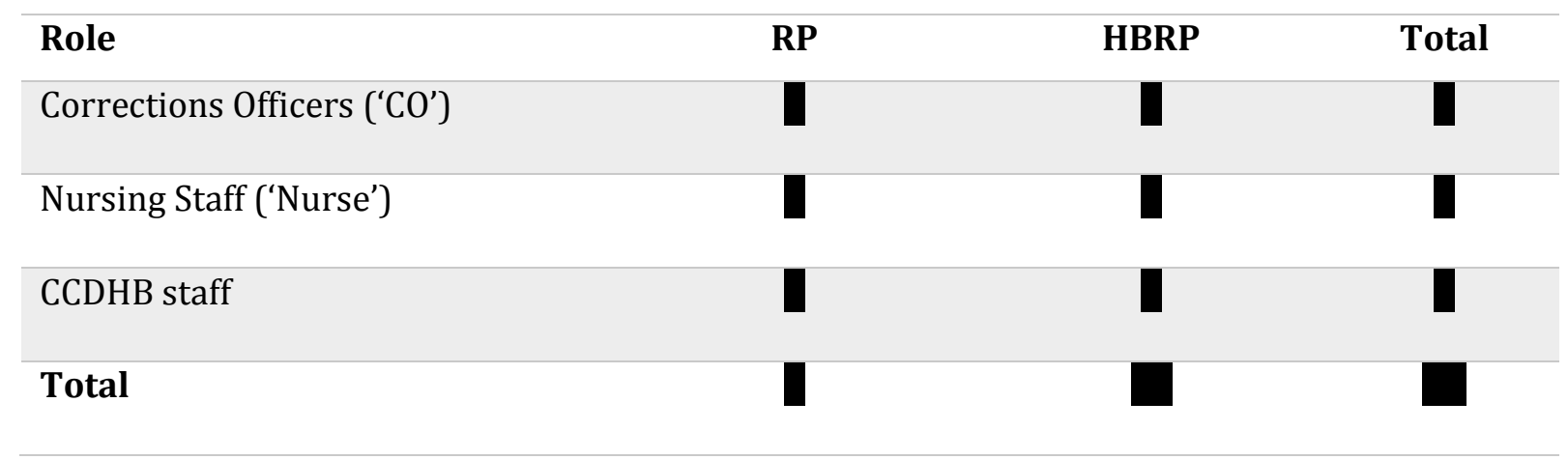

Unlike the rest of New Zealand's prison system staff (Department of Corrections, 2014a), a majority of the participants interviewed for the purposes of this thesis were female, as highlighted in Table 2. The participants were also highly European, with no Māori or Pacific staff represented in this study. It is unclear whether such demographics were representative of wider ARU staff; however, the nature of this cohort raises some wider questions about the limitations of the Unit, particularly given the highly gendered and cultural differences involving in responding to mental health problems (Scraton \& Moore, 2002; Baxter, 2008). In relation to this, participants highlighted how specific Māori cultural and spiritual needs were not always immediately recognised within the ARU, which were often conceived to be mental health issues until identified and addressed by an onsite Māori spiritual advisor. Similarly, staff commented on how culturally-specific communication norms, such as the use of minimal eye-contact when speaking to members of the opposite sex and elders among Māori, were misinterpreted and viewed as 'at-risk' factors by staff, further reinforcing the importance of cultural understandings within the ARU environment. Further, the somewhat misrepresentative demographics of the participants involved may place some implicit limitations on this thesis more directly, in terms of the breadth and scope of staff views and perceptions presented. 


\begin{tabular}{|lcc|}
\hline Gender & N & \% \\
\hline Female & 12 & $75 \%$ \\
\hline Male & 4 & $25 \%$ \\
\hline Nationality & 13 & $82 \%$ \\
\hline NZ European & 3 & $18 \%$ \\
\hline Other & Mean & Range \\
\hline Age & 46 & $30-69$ \\
\hline Years of experience & & $1-14$ \\
\hline
\end{tabular}

\section{Recruitment and interview schedule}

My original application outlined that research information flyers would be distributed in ARUs and Health Centres of both prisons, to recruit participants and inform staff of the research project well in advance of my arrival. This proposed method of recruitment would ensure that participation was voluntary, in line with ethical principles of informed consent (Patel et al., 2003). My flyers (Appendix E), approved by the Research Team, were passed on to the Regional Health Manager, who then passed them for Unit distribution. Unfortunately, for unknown reasons, the flyers were never circulated in either prison. This affected recruitment processes and, while interviewees were able to withdraw at any stage, I cannot be sure that participation was entirely voluntary.

In HBRP, the Principal Corrections Officer (PCO) and the Health Centre Manager informed me that they had selected a random sample of interview participants, based on rostering and availability. Where possible, I approached selected staff prior to interviews, to answer questions and to ensure they were happy to take part. Four participants were selected in this manner. In RP, I recruited participants via opportunistic sampling (Matfin, 2000); the Health Centre Manager and PCO supplied me with all names of staff rostered to work over the following weeks, upon arrival to the prison. I directly approached, or in some cases was introduced to, these staff within the

\footnotetext{
7 Demographic information pertaining to two participants from RP could not be obtained, despite the efforts of the researcher.

8 Working for either the Department of Corrections or for the DHB (in a forensic capacity).
} 
Unit and asked if they would be happy to take part in the research. I then arranged interview times and locations. While my initial proposed recruitment method did not go according to plan, the interviews still provide a diverse range of perceptions and experiences among staff.

My proposed interview schedules received full sign off from the Research and Evaluation team, following some minor amendments (Appendices F, G and H). The semi-structured interview schedule allowed me to address all of the key research themes, but encouraged some flexibility. This allowed conversations to depart from the schedule, and sometimes lead me to new, useful ideas that I had not previously considered.

\section{Entering the prison}

Prior to the interviews, a considerable amount of administrative work was required. Visitor approval forms, approval forms for electronic equipment (laptop and electronic recorder) and an Approved Provider Prison Entry (APPE) Card all needed to be processed and signed, and shown each time I entered the prison. These administrative requirements took around one month to finalise, before I was able to arrange interviews.

Interviews were conducted between February and April 2015. I flew to Hawkes Bay for two 3-day trips in late February 2015 for the first phase of interviews. On arrival at the prison, I received a security induction by the ARU PCO, who showed me the Unit, Receiving Office and Health Unit. In total, I spent six full days conducting interviews with staff members within the prison. In mid-March 2015, I made four trips out to RP for the second set of interviews. Upon arrival, I also received an induction of the ARU and Health Unit from the nursing Team Leader. Upon arrival at both prisons, as per the experience of many prison researchers, I was subject to electronic searches at each prison re-entry and exit (Reiter, 2014).

\section{Data collection and analysis}

I requested 1.5-hour semi-structured interviews with each staff member. Participants were interviewed once, and most interviews ran for the full 1.5 hours. Interviews were 
digitally recorded, to ensure a smooth flow to the conversation and for ease of later analysis (Clough \& Nutbrown, 2007).

In HBRP, interviews were conducted in the PCO's office, located in the hub of the prison's ARU. At times I felt that this proximity to other staff members impacted on confidentiality especially given that the door, which was situated very close to the ARU'S staff pod, did not fully close. On one occasion a participant whispered their answers and expressed reluctance in answering questions, as they wanted to avoid staff members overhearing. This was particularly problematic when responding to questions about the effectiveness of MDT workings and perceptions of other ARU staff.

At RP, interviews took place in a small office, down a corridor directly adjacent to the ARU. The office was further enough away from the central ARU hub to ensure privacy. Interviews with forensic staff took place in offices at Te Korowai Whariki (Regional Forensic Mental Health Services) in Porirua, Wellington, at the request of the staff involved.

Conducting prison research is 'always secondary to the safety and security of the prison and its established routines' (Pautenaude, 2004: 87). I experienced this sentiment first hand, with a number of interviews being interrupted, occasionally for long periods of time, while staff members attended to incidents or requests to assist other workers on the floor. Management staff had prepared me for this eventuality and I worked around these interruptions the best I could.

\section{Presentation of self}

Given the traditionally male-dominated culture of prisons, female researchers can face unique barriers when entering and engaging with the prison system, particularly in male prisons (Gurney, 1985). As a young woman with no experience in prison research, I did at times find myself feeling somewhat 'thrown in the deep end', especially during initial fieldwork stages. For example, one interview participant stressed to me that as a female she didn't feel particularly safe working in the ARU, given the volatile and mentally unwell nature of many prisoners. This startled me somewhat, as I was conducting interviews in plain sight of prisoners who were milling about the unit. While 
I was more aware of my gender than I usually might be on the 'outside', I was never subjected to any rude remarks or cat-calling when around anyone in the prison.

My status as a researcher was also questioned in other ways. During the course of one interview, one participant highlighted my position by saying, in a passing remark, '...you're a complete outsider, forgive me, you probably haven't got a clue what goes on in here' (CO, HBRP). While my status as an outsider could be perceived as a 'challenge', I believe it was a useful tool as it meant I avoided 'choosing sides' between participants an issue that other prison researchers have faced (Liebling, 2001b). Approaching the work openly as an outsider, with no apparent pre-conceptions about the ARUs management, allowed for trust to develop. Further, Gilbert (1994) has noted that a certain level of naivety and humility on the part of the researcher can also be conducive to more active participation among those involved, which I agree with. Stressing my non-affiliation with the Department, and their priorities, also helped to foster levels of trust among participants. In sum, my inexperience conducting prison research, and my relative naivety, while challenging at times worked favourably for this research and allowed for open discussions and a breadth of experiences to be discussed and used as part of this thesis.

\section{Analysing and finalising the research}

All interviews, bar one, were transcribed verbatim. One interview recording became corrupted and was subsequently lost; in that instance, rather extensive notes that were taken during the interview were drawn on to substitute. Once transcribed, interviews were codified using NVivo software as a means of identifying and assessing emerging themes. Guided by a semi-structured interview process, themes and categories emerged from 'the ground' up, a form of deductive analysis (Croucher \& Mills, 2014). Data was then further analysed via the use of grounded theory, which comprises a systematic process of gathering and analysing data; developing 'codes' to assist with categorisation and identification of themes and applying a flexible theoretical approach, built progressively into data as it is collected (Glaser \& Strauss, 2012). Given its flexibility, and its encouragement to constantly interact with the data at hand, grounded theory provided a useful base for the exploratory and interpretive nature of this research, particularly for new researchers (Urquhart, 2013). In line with this concept, memos and 
notes were written between coding and writing as a way of understanding and presenting the most important and recurring themes, guided by existing literature on relevant prison practice and theory. Comparisons were also made, within themes, which allowed for greater depth in analysis and for subtleties in the findings to emerge.

My final thesis was subjected to review by the REGC prior to examination and release into the library, as per the initial Research Agreement. A representative from the REGC contacted me ten weeks after formal submission and approved the thesis as the 'final agreed Document'. However, the REGC asked me to delete all quotes from the thesis. After some negotiation between myself, the REGC and my supervisor, it was agreed that four quotes would be removed from the final thesis. ${ }^{9}$ The redacted quotes related to non-compliant use of control and restraint, the practical impact of risk-aversion on ARU practice and views of prisoners. It was also agreed that data relating to participant demographics (Table 1, page 14) and ethnic identity (Table 2, page 15) would also be removed, to protect the identity of individuals.

Despite these elements, the thesis is an important contribution to the wider, and somewhat scant, literature unpacking the role of multi-disciplinary teams and the positive impact that healthcare teams can have on enhancing care-oriented practice in prisons. The thesis, and its findings, may be a useful point of reference for the Department of Corrections to consider, should the policy and practice of ARUs be reformed or developed in the future and provides useful insight to the practical operational realities of the Unit, across two correctional sites.

${ }^{9}$ See pages $54-55 ; 67 ; 69-70 ; 74$. 


\section{Chapter Three: Conceptualising a Care-Oriented Approach to 'At-Risk' Prisoners}

While self-harm and suicides are multi-faceted issues, with no 'quick fix' or easy solutions, the policy surrounding 'at-risk' prisoners is obliged to uphold minimum rights standards and provide humane treatment to those in need. This chapter signposts some key normative standards in responding to 'at-risk' prisoners including emerging debates around care-oriented correctional practice in relation to suicide prevention. The influential role that prison staff can play will also be emphasised as a central platform for developing more holistic, humane response to 'at-risk' prisoners (Skogstad et al., 2005). Given the ARU framework sits within a wider correctional complex, the chapter will also highlight some inherent systemic or institutional factors and priorities that may run counter to the provision of care 'on the ground' and which should be borne in mind as part of reframing the current ARU approach.

\section{Suicide and self-harm in prison as complex phenomena}

Suicide is a multi-faceted, ambiguous and complex phenomenon, both in correctional settings and in the wider community (Daniel, 2006; Ministry of Health, 2006). In the penal context, suicidal and self-harming behaviours are often depicted as the result of pathological issues or individualised deficiencies among prisoners (Medlicott, 2001); studies show that demographic factors relating to suicidal behaviour are concentrated within prisons, with prisoners constituting an inherently 'at-risk' or 'suicide-prone' group (David \& Codd, 2010: 90). Their heightened suicide risk is linked to factors of being young and male, while also connected to high rates of drug and alcohol addiction, family breakdown or experiences of sexual and physical abuse (Simpson et al., 1999; Borrill et al., 2005; Daniel \& Fleming, 2006). Clinical factors, such as mental health issues, are also leading risk factors for suicidal or self-harming behaviour (Fazel \& Danesh, 2002). These problems are often exacerbated by inadequate mental health services and limited resources available in prison, which are not equipped to properly address prisoners' underlying needs or aid longer term recovery (Roguski \& Chauvel, 2009). 
The prison environment and other institutional factors have been found to further elevate the risk of self-harm and suicide in prison, not found in the general population (Barker et al., 2014). Studies have shown the experience of imprisonment in itself can be detrimental to an individual's mental well-being (National Health Committee, 2010). For instance, poor coping methods and feelings of shame or guilt among prisoners are attributed to an increase in suicide or self-harm risk (Daniel \& Fleming, 2006). Many prisoners experience bullying (from prisoners and staff), are unable to cope with 'prison life' and express feelings of loneliness and isolation (Liebling, 2006; Sharkey, 2010). Lack of staff supervision, sensory deprivation, isolating conditions and overcrowded units have also been linked to suicidal and self-harming behaviours among prisoners (Daniel, 2006; Owers, 2006). The impact of this wider correctional context cannot be overlooked, particularly when reflecting on the overall purpose and use of ARUs as a way of preventing self-harming behaviour. Further, this range of factors, all of which are unique to the prison environment, highlight the complexity of developing and enacting suitable policy responses to those deemed 'at-risk'.

\section{Legal obligations relating to care-oriented responses to 'at-risk' prisoners}

A central objective of this thesis is to explore some of the opportunities and limitations for care-oriented practice to be administered to 'at-risk' prisoners, with particular focus on the input and role of staff. Before exploring the current conceptual underpinnings of the ARU framework in more depth, it is important to lay out what a care-oriented response to 'at-risk' prisoners constitutes for the purpose of this thesis. Further, it is important to note that most policies surrounding 'at-risk' prisoners are obliged to uphold minimum rights standards and provide medical treatment, where mental health issues exist.

\section{'Humane containment' and equivalence of care}

One element of care-oriented practice relates to the notion of 'humane containment' or equivalence of care. In a broad sense, the philosophy of 'humane containment' calls for minimal security-based or physical restrictions and an environment which encourages 'normal' community standards of living that will minimise feelings of isolation, disempowerment and detachment for those detained (King \& Morgan, 1980: 37). With suicide and self-harm prevention commonly falling under the wider ambit of mental 
health care provision (Fazel \& Danesh, 2002), a humane approach recognises that prisoners should have the same right to access and quality of healthcare as the general population (Corcos \& Lewin, 2001). In New Zealand, the philosophy of 'humane containment' has been enshrined in relation to the provision of medical care, to some extent, in legislation:

A prisoner is entitled to receive medical treatment that is reasonably necessary... The standard of health care that is available to prisoners in a prison must be reasonably equivalent to the standard of health care available to the public (Corrections Act 2004: Section 75).

While providing a useful normative framework, such provisions may be compromised in practice. For example, previous Corrections Minister Anne Tolley recently stated that while prisoners do receive a good standard of care, they 'shouldn't get a better health service being in a prison than hard working law-abiding citizens' (Radio New Zealand, 2013). Despite these shortcomings, as a guiding philosophy, 'humane containment' prioritises holistic, tailored responses that preserve identity, 'self' and well-being above shorter-term preventative mechanisms, such as the management of risk via seclusion (Coid et al., 2001; Morgan et al., 2007). In principle, prisoners can and should expect to receive the same human rights as those living in the general community, despite automatically forfeiting a number of freedoms when entering prison.

\section{Adherence to human rights standards}

The philosophy of 'humane containment' is also practically embedded in a number of normative human rights standards. Some regions have legal mechanisms which uphold and protect prisoners' rights, for example the European Convention on Human Rights (Genders \& Player, 2014). In this context, some of the more extreme preventative or containment based approaches to suicide prevention, such as the use of shackles or handcuffs to prevent individuals from harming themselves, have been legally challenged on the grounds that they amount to inhumane or degrading treatment (Dudley et al., 2012). In the context of ARUs, key human rights standards - namely a prisoner's right to life, right to adequate healthcare and right to be treated with humanity and respect - all come to the fore when conceptualising the administration of care-oriented approaches to 'at-risk' prisoners. 
Correctional systems worldwide are legally responsible for the welfare of those held in their custody and have a duty to protect prisoners from unnatural and preventable deaths, including suicide (Livingstone, 2000; Martynowicz, 2011). Despite ARUs being viewed unfavourably by some (Camilleri et al., 1999; Scraton \& Moore, 2005; Stanley, 2011; Wakem \& McGee, 2012), the existence and development of such units was originally underpinned by the work of human rights groups, who advocated for better protection of prisoner's 'rights to life' (Cliquennois \& Champetier, 2012). Internationally, provisions relating to the 'right to life' 10 have been interpreted by the European Court of Human Rights as placing a positive or active duty on the state to protect the lives of those who are at risk of suicide (Leach, n.d.; Keenan v. UK, 2001). Previously, rights were viewed as justifiably limited if they are viewed to be 'necessary and proportionate' (EHRC, 2012: 46). As Kaufmann (1999) summarised:

where measures are imposed for security, disciplinary or protective purposes [including protection for suicide], the [Court] has shown a remarkable tolerance, irrespective of the effects of their stringency and the effects on the health of the victim (Kaufmann, 1999: 457).

However, this approach seems to be waning in respect of prisoner suicide. Prison officials are now required to go beyond the provision of 'blanket measures' of physical restraint or intrusive surveillance in order to safeguard those at risk of suicide (Genders \& Player, 2014: 444). In the European context, the 'right to life' now obliges prisons to provide supportive relationships, adequate healthcare services and to uphold respect for a prisoner's private life, personal autonomy and physical integrity (Genders \& Player, 2014; Livingstone, 2000). Where mental health concerns are present, failure to provide adequate healthcare while segregated was found to constitute inhuman and degrading treatment (Keenan $v U K, 2001$ ). In these instances, the interpretation of 'right to life' by the European Court of Human Rights highlights an expectation for the state to consider the individual needs of prisoners and to provide much more than the basic necessities of life, in a care-oriented manner.

Similar 'right to life' obligations exist in New Zealand and are enshrined by section 8 of the New Zealand Bill of Rights Act 1990 (NZBORA) and via our ratification of Article 6 of

${ }^{10}$ Specifically, Article 2 of the European Convention of Human Rights. 
the United Nations International Covenant on Civil and Political Rights (ICCPR) $1966^{11 .}$ However, unlike in Europe, 'such provisions have lain dormant in [our] system' and have not been practically utilised or developed to the same extent in domestic courts (Juss, 2014 as cited in Clark, 2014). While not binding, New Zealand is often mindful of international developments when considering the domestic development of analogous legal provisions (Clark, 2014). Developments towards heightened obligations on the part of the state in respect to prisons, as well as their procedural nature, are useful points when considering a shift towards more care-oriented penal practice.

Despite this 'gap', the New Zealand state must still take all reasonable steps to ensure an individual's care, safety and wellbeing is upheld in a similar way via 'duty of care' obligations, which are enshrined in both civil and criminal law ${ }^{12}$ (IPCA, 2015). In short, civil, criminal and other remedies can be sought if a reasonable standard of care is not provided and if an individual under state custody is harmed as a direct result (IPCA, 2015). Any unnatural deaths in custody prompt Coroners' and Corrections inspector inquests, as well as independent investigations led by the Ombudsman, which aim to highlight systemic deficiencies that may give rise to negligent or unsafe practice. These obligations prompt officials to act with greater caution, care and oversight when managing individuals who may be 'at-risk', to avoid legal fallout or public criticism (Martynowicz, 2011).

As previously noted, the duty for a state to safeguard a prisoner's life can bring in secondary obligations regarding access to healthcare. In respect of suicide and selfharm in prison, upholding 'right to life' requirements while also protecting a prisoner's physical, mental and moral integrity can be a challenging balance to strike. In some circumstances, including instances of suicide, withholding necessary access to medical care in prison has been viewed as a form of torture, inhuman or degrading treatment or punishment, as per Article $3^{13}$ of the ECHR (Livingstone, 2000; Mendez, 2013). A recent report by the UN Special Rapporteur on Torture further highlighted that 'nontherapeutic' medical treatment may be in breach of Article 3. Such non-therapeutic

\footnotetext{
11 Note the Covenant was ratified with some reservations withheld.

12 Namely section 317 of the Accident Compensation Act 2001 and Part 8 of the Crimes Act 1961 (specifically, sections 145, 151, 171, 190 and 195).

13 'Prohibition of Torture: No one shall be subjected to torture or to inhuman or degrading treatment or punishment.'
} 
responses include indefinite detention, the use of restraint and seclusion, and segregation (Mendez, 2013:16). According to the UN report, a 'lack of resources or services' cannot alleviate a state's obligations to provide healthcare (Mendez, 2013:20).

In relation to this, the ICCPR, ratified by New Zealand, requires 'all detained persons to be treated with humanity and respect for their dignity' (UN Generally Assembly, 1966: Art. 10). Section 23(5) of the NZBORA also states that where persons are detained by the state they are to be treated with humanity and respect. Internationally, the incorporation of these rights into official suicide and self-harm prevention policies preempts a care-oriented approach to prisoner treatment and can uphold a prisoner's right to personal autonomy. Examples in policy include recognizing that 'at-risk' prisoners, specifically, have rights to be free from 'intrusive surveillance' or unnecessary removal of clothing (EHRC, 2012: 34).

In sum, many human rights and other normative legal standards are underpinned by philosophies that promote equivalence of care and care-oriented practice more generally. In the context of prisoner suicide and mental health, some of these safeguards have been interpreted as placing a positive and procedural duty on the state to not only prevent death from occurring, but also to provide treatment and care, when relevant, and to protect a prisoner's sense of autonomy and integrity. In New Zealand, such standards and frameworks have been subject to less direct legal application and scrutiny when considering the status of 'at-risk' prisoners; however, overseas developments should be considered when looking to the future of 'at-risk' practice and policy. While normative standards are only 'one piece of the puzzle', in terms of the practical embodiment of care-oriented practice, they are grounded in positive philosophies that further make a case for progression in current ARU policy. As discussed in more depth below, a 'culture of rights' among staff working 'on-the-ground' is equally, if not more, important to consider and should be read in light of such frameworks and standards (Genders \& Player, 2014: 437). 


\section{The current ARU system: criticisms and progressive policies}

International best practice standards dictate that there is a viable place for 'suicide-safe environments', where prisoners are held in cells with minimised hanging points ${ }^{14}$ and are restricted from accessing 'lethal materials', as a way to prevent self-harm and suicide (WHO, 2007: 16). In many Anglophone jurisdictions, endorsement for this approach has been translated into and legitimised ARU policy and practice (as evidenced in New Zealand's use of ARUs). Australian states, such as Victoria (VIC) and New South Wales (NSW), have also adopted similar policies to manage 'at-risk' prisoners, via their utilisation of 'Safe Cells' and 'Safe Observation' cells which have minimised ligature points and furniture (Corrections Victoria, n.d.; Corrective Services NSW, n.d.). Other restrictions, including the use of anti-suicide gowns and curtailing prisoner access to razor blades, forms part of many Western jurisdictions' wider antisuicide policies (Department of Corrections, 2013a; Corrections Victoria, n.d.; Burrows et al., 2003). At the more severe end of the continuum, in some jurisdictions including New Zealand, physically intrusive control and restraint mechanisms, such as the use of tie-down beds, are endorsed in 'extreme' cases where risk of suicide is high (Victorian Ombudsman, 2014; WHO, 2007; EHRC, 2012). Reports have described prisoners in the United States being chained to beds and forced to wear 'leg irons' to prevent selfharming behaviour (Livingstone, 2000). While not the sole response to 'at-risk' behaviour, these developments highlight how minimising immediate environmental risks has become the dominant model for mitigating the risk of self-harm or selfinflicted death among prisoners.

\section{Criticisms}

Despite protecting life, the use of isolation cells has been widely condemned by researchers and officials alike, for a number of reasons ${ }^{15}$ (Camilleri et al., 1999; Scraton \& Moore, 2005; Stanley, 2011; Wakem \& McGee, 2012). Placing a suicidal prisoner in a depriving environment, with little to no external stimuli, can reinforce feelings of isolation, hopelessness and anxiety (National Health Committee, 2010). As an Australian advocacy group described NSW's safe cells:

\footnotetext{
${ }^{14}$ Due to the fact that a majority of prison suicides are due to hanging (WHO, 2007; Lyneham \& Chan, 2012).

${ }^{15}$ Criticisms specific to New Zealand's ARU framework are expanded on in Chapter Four.
} 
Low and depressed? Oh, well we better stripsearch you and isolate you in the most oppressive environment imaginable. Feeling better now...? (Doyle, 1997).

Analogous cells in Northern Ireland have been described as prioritising the 'appearance of normality', by preventing all means for prisoners to self-harm, above the restoration of sound mental well-being (Scraton, 2007: 47). In that context, the daily experience of 'at-risk' prisoners was described as one of anguish and deprivation, rather than positive engagement (Scraton, 2007). Many prisoners resent being held in ARUs and cite feelings of 'disempowerment, fear and despair' (Bell, 1999: 725). The distressing and often involuntary experience of being held in an ARU can also stop prisoners from disclosing personal issues or troubles to prison officers, limiting the opportunity for staff to respond in a care-oriented manner (Liebling, 1992).

In a similar vein, healthcare professionals have criticised the placement of prisoners in ARUs, noting how such an environment can actively contribute to a deterioration in mental state, particularly among those with pre-existing mental health conditions (Anasseril, 2006). Concerns have also been raised about the frequent and inappropriate use of ARUs for severely mentally unwell prisoners, in instances where secure beds in psychiatric hospitals are unavailable (Coid et al., 2001). While some healthcare professionals consider ARUs to be legitimate in some respects, for example when used for short-stay 'time out' or 'cooling off' periods (Corcos \& Lewin, 2001), many have noted that the provision of care is inherently constrained by the restrictive nature of ARUs (Bell, 1999). When discussing ARUs, they have outlined that 'confusion exists over what comprises a preventative intervention with therapeutic intent and what might be construed as a punishment', given their similarities to solitary confinement (Coid et al., 2001: 321). In a more practical sense, prison-based healthcare professionals have noted with frustration that placement in an ARU is often the only option available when responding to 'at-risk' prisoners (Bell, 1999; Corcos \& Lewin, 2001).

\section{Progressive policies}

Some jurisdictions have seen progressive, albeit subtle, shifts in philosophy and practice within similar existing ARU frameworks (Victorian Ombudsman, 2014; Burrows et al., 2003). Internationally, policies have come to recognise that less dehumanising physical environments within the prison, in which sufficient levels of care and human interaction 
can be administered and exist, work positively to lessen the chances of self-harming or suicidal behaviour (Wortley, 2002). Importantly, Wortley (2002) notes that, in the context of suicide prevention, the use of physically restrictive cells is not entirely necessary. Others, such as Reser (1992) have also stressed how 'deinstitutionalising' the environment for 'at-risk' prisoners can be positive in minimising suicide or self-harm risk. This can be aided by the increased provision of natural lighting, radios, access to drinking water and use of soft furnishings. According to Reser (1992: 174) such an approach can alleviate a prisoner's sense of containment and can help to relieve the 'cumulative and stressful lack of control', a feeling common among suicidal or selfharming prisoners.

In relation to these developments, some policies are increasingly recognising that 'ligature-free'16 environments do not necessarily need to be totally void of stimulus and human interaction in order to prevent death and other harm. The WHO's (2007) suicide-prevention in jail guidelines also note that while physical restraints and surveillance may be appropriate in acute circumstances, such intrusive restrictions should only ever occur alongside active and frequent interactions with staff, particularly mental health practitioners. In extreme cases, where it is necessary to segregate or isolate a suicidal prisoner, provisions should also be made for uninterrupted supervision and human contact. As noted by Anasseril (2006: 170), who writes from a mental health perspective:

Frequent monitoring of inmates in cells is more important than any cell design. Nothing can replace human supervision as a deterrent to suicide.

In line with this thinking, in England and Wales, stark 'strip cells' were eliminated from the Prison Service in 2000 and replaced by 'Safer Cells' (Burrows et al., 2003). The primary purpose of 'Safer Cells' remains 'mak[ing] the act of suicide or self-harm as difficult as possible', via reduced ligature points (Burrows et al., 2003: 17). However, Safer Cells are also designed to create a 'normalising, light, airy environment which serves to reduce the stress levels of those placed in them' (Burrows et al., 2003: 17). Safer Cells aim to provide comfort and include inbuilt TVs, privacy screens and storage space for prisoners to keep their personal belongings. An evaluation of the new

\footnotetext{
16 Ligature points are any environmental feature which could be used to support a noose or other strangulation device for the purpose of attempting suicide.
} 
framework found that, while isolating prisoners was still not ideal practice, Safer Cells provided a less dehumanising alternative environment for prisoners, in extreme circumstances (Burrows et al., 2003). The Safer Cell policy is also complemented by regime activities that are available to 'at-risk' prisoners (e.g. chaplaincy, visits, gym, learning/skills places), recognising that involvement in activities can 'significantly reduce a prisoner's risk of harm to themselves' (UK Ministry of Justice, 2013: 34). Levels of observation can be reduced if a prisoner takes part in out-of-cell activity and regime activities can also be organised to take place in-cell, if required.

In their recent report, exploring deaths and harm in custody, the Victorian Ombudsman (2014) highlighted the divergence in approaches between the adult and juvenile custodial jurisdictions, the latter providing a more therapeutic and rehabilitative approach to care. For example, Victoria's youth justice facilities allow prisoners to be held in their own cell and to interact with other people in the unit (Victorian Ombudsman, 2014). The report highlights that the traditional restrictive method of responding to adult 'at-risk' prisoners 'raises questions as to whether this practice can be detrimental to the mental health of some prisoners in the long-term' (Victorian Ombudsman, 2014: 60). In the adult jurisdiction some emerging good practice should be noted. For example, prisoner listeners or peer supporters ${ }^{17}$ are used to associate with prisoners who are under strict observation, in order to reduce their social isolation (Corrections Victoria, n.d.). Similarly, the Scottish 'at-risk' allows prisoners to remain in their own cells during the day and in their own clothing. This approach is underpinned by a desire to maintain the prisoner's 'sense of control and familiarity with their surroundings', minimising risk without compromising physical integrity or autonomy (National Health Committee, 2010: 57).

In sum, the existence and utilisation of ARUs in New Zealand is underpinned by a desire to uphold 'right to life' requirements and, more generally, to keep prisoners safe. The benefits of ARUs have been recognised and their use has been endorsed by reputable agencies such as the WHO (2007). Despite this, their use has been widely criticised, with their isolating, non-therapeutic nature being framed as particularly problematic. In response to such commentary, there have been advances in similar jurisdictions to reform ARUs to enhance a more care-oriented approach to practice. Such developments

17 Peer support services are provided by internal prison workers and by the Australian Red Cross. 
include greater prioritisation of inclusion, by normalising communications and via the introduction of activities. In some jurisdictions, equivalent ARUs have also modified the physical environment to enable a less punitive, solitary ARU experience. While these shifts have been relatively limited in their approach, and can by no means be described as revolutionary, such steps could be considered as a way of incrementally developing care-oriented practice within New Zealand's current ARU framework.

\section{The role of staff and multi-disciplinary workings within ARUs}

\section{Positive contributions of staff}

While policies certainly form an important part of ARU practice, a key focus of this thesis is to explore the extent to which multi-disciplinary staff can enhance careoriented responses to 'at-risk' prisoners. When staff are trusting and supportive towards 'at-risk' prisoners, they can make effective contributions to prisoner well-being and can help to prevent suicide risk (Borrill et al., 2005). Staff are also well positioned to notice changes in a prisoner's behaviour and are often the first point of call for those seeking help (Rowan, 1994). Their role in recognising potential motivators or risk factors can make 'the single most important contribution in suicide prevention' (Liebling, 1992: 219). Prisoners are unlikely to disclose personal concerns, or concerns about others, unless they feel comfortable placing their trust in prison staff. By taking the time to listen to a prisoner's concerns they allow prisoners to 'feel heard' and have their issues taken seriously, which can be a therapeutic experience in itself (Pannell et al., 2003). Staff can also refer prisoners to specialist health services, acting as the gatekeepers to individualised care and longer term recovery, where needed. In order to sustain good relationships, staff must take a proactive approach to ensuring lines of communication are kept open (Pannell et al., 2003).

\section{Training and resourcing}

The ARU is undoubtedly a challenging working environment. Prison staff must be constantly alert when responding to high-needs prisoners, such as those with severe mental health problems and those actively attempting to harm themselves. They also have to respond to verbal and physical intimidation, to themselves and other prisoners. It is perhaps unsurprising that the everyday challenges of the prison as a working 
environment can foster high turnover and feelings of burnout and frustration among staff, who may feel undervalued or under-resourced (Stanley, 2011).

In order to minimise these challenges, and to facilitate a professional culture of cooperation and 'excellence', good training to appropriately recognise and respond to suicidal risk factors is crucial (Anasseril, 2006; Liebling, 2006; WHO, 2007; Stanley, 2011: 111). Staff who lack the requisite confidence or skills to deal with high-needs prisoners run the risk of 'automatically channelling' prisoners into ARUs, in order to eliminate any possibility of risk (Roguski \& Chauvel, 2009:12; Liebling, 2006). Lack of training can limit the positive impact of inter-personal relationships, when managing suicidal prisoners (Anasseril, 2006). Effective training can also help to encourage collaborative working practices between multi-disciplinary staff working alongside each other (Anasseril, 2006).

Adequate resourcing is also important. In England and Wales, the ability for staff to respond therapeutically to prisoners has been directly inhibited by restructuring and downsizing, in response to recent budgetary cuts (Genders \& Player, 2014). The Chief Inspectorate of Prisons has linked the reduction of staff numbers to the recent significant increase in the number of prison suicides in England and Wales (HM Inspectorate of Prisons, 2014). While New Zealand has taken a number of active steps to support, upskill and retain staff (Stanley, 2011), the relevance of training and resourcing staff cannot be undermined or ignored.

\section{A multi-disciplinary approach}

On the whole, a multi-disciplinary approach involves greater input and responsibility of healthcare staff, who work alongside custodial staff to manage and assess risk, provide medication and refer to other services (HM Inspectorate of Prisons, 2007). A major point of contention has been the relationship between the public health and prison health system and how the structure of this relationship can best serve the needs of prisoners (King, 2012). In relation to this, the development of multi-disciplinary ARU policies have frequently been guided by high-level collaboration at an agency level. In NSW, for example, Mental Health Units have developed from close collaborations between the Justice and Forensic Health Network (Justice Health) and the Department of Corrective Services (Corrective Services NSW, n.d). 
In England and Wales, multi-disciplinary developments were underpinned by large scale agency shifts, namely the National Health Service's (NHS) take-over of responsibility for prisoner health in 2004. This change sought to respond to increasing recognition that the health care culture [in prison] was influenced by traditional attitudes, with an emphasis on security and less on nursing practice and health improvement' (HM Prison \& NHS Executive, 1999: 11). Authorities were concerned about the quality and standards of prisoner health care, which varied greatly across prisons and which offered little professional support to healthcare staff (Sim, 2002). Following this shift, new policy on the collaborative management of 'at-risk' prisoners known as Assessment, Care in Custody and Teamwork (ACCT) was introduced as a joint Department of Health/Prison Service initiative (Sedenu, 2005). This shift towards enhanced multi-disciplinary working led to the introduction of increased mental health training for custodial staff, the creation of a self-harm network to share good practice across disciplines and requirements for external healthcare staff to generally play a more active role in monitoring and treating 'at risk' prisoners (Sedenu, 2005). While important, in the interests of scope, agency level tensions around oversight of correctional healthcare will not be discussed in extensive detail here. However, in the context of ARUs, ground-level tensions and perceptions among staff in respect of custody and care priorities is central to developing a care-oriented response.

\section{Multi-disciplinary workings: custody vs. care roles}

Medical and custodial staff members can make positive contributions to those at risk of self-harm and suicide in prison (Sharkey, 2010). Positive co-operation between staff members, and strong working relationships, is an integral element of suicideprevention in prison (WHO, 2007; Short et al., 2009). However, despite the obvious benefits, both custodial and health staff working within ARUs can face ambiguity and conflict in respect of their roles and responsibilities (Liebling et al., 2011). Studies have exposed that many prison officers feel their duty to provide security conflicts directly with their duty to provide care (Liebling, 1992). In a similar vein, prison officers have expressed concern about lack of guidance when dealing with self-harming prisoners and they struggle to balance competing institutional demands, such as 'supervisor, custodian, disciplinarian, administrator, observer, manager or mentor' (Short et al., 2009: 409). In relation to this, officers who take a security-driven approach are more 
likely to engage in conflict with and develop negative views of prisoners (Williams, 1983). On the other hand, correctional staff who take a more therapeutic approach with prisoners have been criticised by peers as being weak or soft (Sim, 2002).

Similarly, health professionals working in a correctional environment face unique challenges of their own. Research examining the role of prison health care staff highlights feelings of professional isolation in the course of their employment (Birmingham, 2003). Further, while one may assume that a greater healthcare presence would equate to a more therapeutic environment, medical staff can lose sight of their 'care' functions and get swept up in the security-driven ethos of the prison, particularly when employed directly by the prison (Sim, 1990). This thesis is interested in exploring how these issues are grappled with and how, in light of these conflicts, MDT relationships can contribute to care-oriented approaches for 'at-risk' prisoners.

\section{Systemic and institutional barriers to care-oriented practice}

While care-oriented responses to prisoners are desirable, both in ARUs and across the wider correctional environment, some have argued such an approach runs counter to correctional priorities of risk-management and security (Sim, 2002). Similarly, where care-oriented principles have been incorporated into policy, it is clear that prison culture - the relationships or values shared by staff - also has a major role in shaping policy interpretation and subsequent practice (Cox, 1984; Liebling, 2004). These factors should be kept in mind when considering the possibility of developing a welfarist response to 'at-risk' prisoners.

Much research has highlighted the impact that prison culture can have on policy interpretation and subsequent practice (Cox, 1984; Liebling, 2004). One reported cultural predisposition is a staff view that prisoners are 'less-eligible', in that they should not receive healthcare or other rights to a superior standard than those on the outside (Sim, 1990:6). As Sim (2002: 307) has reiterated, the 'culture of prison officers is dominated by the view that prisoners are constantly attempting to 'blag' the prison authorities'. Staff may view self-harming prisoners as 'non-genuine', manipulative or acting for personal gain (Pannell et al., 2003: 104). This attitude can have significant implications for care practices as staff minimise a prisoner's distress or emotional state. Viewing prisoners as 'less eligible' or non-legitimate can also actively impede a staff 
member's willingness to help, even towards those who may have more 'legitimate' needs or concerns (Pannell et al., 2003).

Construing prisoners as 'less-eligible' is particularly acceptable in today's penal climate, where managerial, compliance-based frameworks dominate institutional measurements of 'success' or good practice. Increasingly, services and operational projects within public services are measured against frameworks of targets and standards, such as Key Performance Indicators (KPIs) in the UK and Better Public Service (BPS) targets in New Zealand (Liebling, 2004; Department of Corrections, 2011). Some commentators have seen benefits to this approach, arguing that the introduction of such measures allows for government agencies to be more 'reflexive, self-inquiring, future-oriented', and may be better placed to engage in long-term and strategic planning (Liebling, 2004: 57). However, this approach could lead government agencies, including those in the correctional sphere, to prioritise concerns relating to management, accountability and risk aversion at a bureaucratic level, to the detriment of more individualised notions of prisoner well-being (Liebling, 2004; Genders \& Player, 2014).

For example, in New Zealand, central government agencies ${ }^{18}$ review the Department of Corrections against their Performance Indicator Framework (PIF) (SSC, the Treasury \& DMPC, 2012), which measures increases and declines of various incidents, assaults and complaints, while pitting progress against international benchmarks. As part of this, prison suicide rates are conceptualised as 'key metrics', with annual declines and increases measured directly against relevant in-house 'services' and annual budgetary standards (SSC, Treasury \& DPMC, 2012). Similarly, measurements of 'success' are viewed simplistically and numerically via declines or increases in annual correctional suicide rates. It is within this context that prisoners at risk of self-harm or suicide 'pos[e] problems for management', rendering the lived experiences and pains of prisoners invisible (Seddon, 2007: 79). In short, the prevention of death at a managerial level is prioritised above any attempts to remedy prisoners' underlying ailments or to treat prisoners as human beings. It is within this context that bare and isolating ARUs can be viewed as legitimate.

${ }^{18}$ State Services Commission (SSC), Treasury and Department of Prime Minister and Cabinet (DPMC). 
While the influence of a human rights framework in guiding operational or policy standards is certainly positive, its existence does not necessarily ensure that 'institutional practices are embedded in a culture of rights' (Genders \& Player, 2014: 437). At the 'ground-level', a commitment to prisoners' individual rights can be 'frequently trumped by competing considerations', such as the management of risk (Genders \& Player, 2014: 438). Risk-centric approaches can undermine the important development of humane, connected relationships with staff, as an emphasis is placed on institutional accountability rather than individual need. Prisoners can feel as though 'they and their problems are fed into the institutional machinery, subsumed into its discourse and transformed into risk', with staff showing limited interest in their longerterm recovery (Crewe, 2011: 517). Given the centrality of risk management and managerialism in this context, compliance to human rights standards can paradoxically provoke poor treatment and a tick-box approach to 'at-risk' prisoners and their treatment.

\section{Summary}

Suicide and self-harm in prison is a complex issue to grapple with and respond to, for both policy-makers and staff working 'on the ground'. Prisoners, by virtue of being in prison, are at an elevated risk of harming themselves and are an inherently vulnerable population group. Traditionally, responding to and preventing self-harm in prison has been very much based on a correctional model, with short-term risk management being prioritised over longer-term recovery and care via the use of segregated ARUs. Such an approach has been criticised as overly punitive and studies have shown that segregation for 'at-risk' prisoners, particularly those with mental health problems, can actually make matters worse in the long-term.

This chapter has highlighted how in recent years, international legal and policy developments have prioritised a new agenda of prisoner care. However, in practice, such shifts are regularly undermined by prison culture, managerial adherence to compliance, limited resources, staffing issues and other challenges unique to the correctional environment. At the same time, it is evident that prisoner well-being can be enhanced by simple shifts in policy, such as by changes to the physical ARU environment. Other positive developments are less inherently wedded in policy and are 
more reliant on positive working cultures and leadership 'on-the-floor', for example enhanced interactions between staff. Such considerations are central to the practical dynamics of care-oriented approaches to prisoner care and are continued in the next chapter, which examines the New Zealand ARU context. 


\section{Chapter Four: The New Zealand 'At-Risk' Framework - Commentary and Practice}

In recent years, New Zealand's prison system has witnessed the expansion of rehabilitative interventions as part of a wider priority to reduce reoffending. In line with this 'rediscovery of rehabilitation', the Department of Corrections has introduced 'therapeutic' treatment and education programmes (Sheffer, 2012: 408; Controller \& Auditor General, 2013: 25). Despite these shifts, the Department's response to suicide and self-harm in prison, specifically within the current ARU framework, remains grounded in discourses of 'managerialism' and risk avoidance (Feeley \& Simon, 1992). This chapter begins with an overview of the current response to self-harm and suicide in New Zealand prisons, before illuminating the on-the-ground realities of ARU practice. These 'real-world' experiences will tie together some of the themes raised above. They indicate that the current focus on managing and controlling multiple manifestations of 'risk', and wider institutional constraints, are key driving forces that shape current ARU practice. These wider forces are central to ARU misuse and overuse, at times subverting or constraining the positive efforts of staff who work within them.

\section{Mental health, suicide and self-harm rates in New Zealand prisons}

Suicide and self-harm is intrinsically linked to prisoner mental health, with mentally unwell prisoners constituting an 'at-risk' group in their own right. Prisoners have a significantly higher incidence of mental health problems in comparison to the general New Zealand population (Office of the Auditor-General, 2008). One explanation for the over-representation of mentally unwell individuals in prisons is in part linked to the widespread closure of New Zealand's psychiatric hospitals in the 1990s, following a number of institutionally damning incidents through the late 1980s (Munro, 2012). Although widespread deinstitutionalisation was not an entirely negative shift, the necessary establishment of community supports for individuals with mental health problems failed to materialise (Munro, 2012). Critics have contended that this was a case of 'throwing the baby out with the bathwater', in which mentally ill individuals were increasingly viewed as posing a danger or threat to the community (Munro, 2012; 
Glaser \& Laster, 1990). In turn, 'prisons have become de-facto institutions' for people with mental illness (Workman, in Munro, 2012: 2).

Since 2001, at least ten New Zealand inquisitorial reports and inquiries have expressed concern regarding the mental health of prisoners (see Ministry of Health, 2001; Robson \& Harris, 2007; Office of the Auditor-General, 2008; Human Rights Commission, 2009; Roguski \& Chauvel, 2009; Stanley, 2011; Wakem \& McGee, 2012). Further, mental health services and treatment options in prison have been described as insufficient (Office of the Auditor-General, 2008; Roguski \& Chauvel, 2009). Other than the introduction of a Mental Health Screening Tool (MHST) in 2012, no major rehabilitative initiatives addressing prisoner mental health have been recently introduced (Department of Corrections, 2013b).

Recent figures show the prevalence of self-harm and suicide in New Zealand prisons. The Department reported that, between 2004 and 2009, 190 prisoners were 'saved' from harming themselves (Brennan, 2010). While the prison suicide rate has dropped significantly in the last 15 years (Brennan, 2010), it still remains high in comparison to the rest of the population. Table 3 comprises information retrieved from the Department for this thesis, providing a breakdown of self-harm and suicide rates in all New Zealand prisons (male and female) over the last decade. More detailed data summaries relating to incidences of self-harm and deaths in custody can be found in Appendix G and Appendix H. 
Table 3: Incidences of self-harm and suicide in New Zealand prisons, 2005-2015

\begin{tabular}{|ccc|}
\hline Reporting year ${ }^{\mathbf{1 9}}$ & Incidences of self-harm & Incidences of suicide \\
\hline $2005-2006$ & 42 & 6 \\
\hline $2006-2007$ & 26 & 5 \\
$2007-2008$ & 31 & 4 \\
\hline $2008-2009$ & 33 & 4 \\
\hline $2009-2010$ & 31 & 9 \\
\hline $2010-2011$ & 23 & 10 \\
\hline $2011-2012$ & 6 & 5 \\
\hline $2012-2013$ & 7 & 3 \\
\hline $2013-2014$ & 19 & 3 \\
\hline $2014-2015$ & 2 & 7 \\
\hline T0TAL & $\mathbf{2 2 0}$ & $\mathbf{5 6}$ \\
\hline
\end{tabular}

The above data suggests that a general downward shift in self-harm events has occurred since 2011-2012, in spite of significant fluctuations, while suicides have remained relatively steady. The Department attributes the decline in self-harm to preventive initiatives, specifically: 'swift action' by staff, as a result of enhanced staff training; the introduction of 'rip-proof' bedding and clothing; the incremental introduction of ARUs across prisons; and new policies, such as the introduction of the New Arrival Risk Assessment (NARA), restricted access to razor blades, and the introduction of the MHST in April 2012 (Brennan, 2010: 1; Department of Corrections, 2013b). The MHST seeks to better identify cases of mental illness among newly-arrived prisoners and provides an extended range of forensic care options to those who positively identify with a mental health issue (Department of Corrections, 2013b). In the year following its introduction, the Department provided over 650 prisoners with ongoing treatment, who previously may have fallen through the cracks (Department of Corrections, 2013b). While New Zealand has seen a decrease in instances of self-harm since the introduction of ARUs a decade or so ago, the correctional suicide rate remains stagnant, suggesting that ARUs are perhaps only able to contain 'surface manifestations' of prisoner distress and prevent harm in the short-term (Cliquennois \& Champetier, 2012: 397).

${ }^{19}$ Reporting year is $1^{\text {st }}$ July to $30^{\text {th }}$ of June. The year 2014-2015 is not comprehensive and runs to 31 March 2015. 
It is worth briefly noting that, despite the introduction of a number of proactive initiatives, fully understanding and accounting for fluctuations in data is fraught with challenges. In the field of self-harm and suicide, inaccuracies of recording are common and can be swayed by changes to reporting practices. Definitions of self-harm and suicide are also open to varying interpretations by staff and officials (Crighton, 2002; Liebling, 1995). For example, self-harm is defined narrowly by the Department as any 'intentional act of harm to oneself which would most probably have led to death if there was no immediate intervention, including all attempted suicides'20. One can deduct from this definition that less 'severe' incidences of self-harm are not represented in the above data - an important omission.

\section{What are At-Risk Units?}

ARUs comprise of solitary cells where prisoners are transferred in efforts to prevent the risk of personal harm or injury to themselves. ARUs operate in 14 out of 19 of New Zealand's prisons ${ }^{21}$, constituting the primary national response to self-harming and suicidal behaviour by prisoners ${ }^{22}$. Interestingly, ARUs are a relatively recent phenomenon, with the first ARU opening in Christchurch Men's Prison in 1997, followed by Units at Waikeria and Whanganui Prisons in 2000. Following this, ARUs were incrementally rolled out across the country, with the most recent ARU opening in HBRP in 2007. Other than the existing current operational policy, little to no information could be retrieved from the Department of Corrections regarding the original policy rationale behind their introduction during this period, nor what existed prior to their introduction ${ }^{23}$.

As well as solitary cells, ARUs also contain 'round-rooms', which are segregated areas designed to manage more violent or disoriented 'at-risk' prisoners. Round rooms differ from ARU cells in that they have no lavatory or running water facilities (Wakem \& McGee, 2012). It should be noted that other types of segregation operate within some

\footnotetext{
${ }^{20}$ Bowman, J. (Personal communication, 20 April, 2015).

${ }^{21}$ ARUs currently exist in all of New Zealand's prisons (private and publicly run), except for Rolleston Prison, Christchurch Women's Prison, Manawatu Prison and Tongariro/Rangipo Prison.

22 Bowman, J. (Personal communication, 13 April, 2015).

${ }^{23}$ Information on the establishment of ARUs was requested from the Department, however little information on their inception appeared to be stored with either the Offender Health Unit or the Information Centre. Some information was retrieved from a Principal Research Advisor and from internal records at HBRP.
} 
New Zealand prisons, for example voluntary segregation cells and management units, used for prisoners deemed to be vulnerable or at risk of harming others (Department of Corrections, 2012c: M.01.03). These are not addressed further here.

\section{Day-to-day operations}

Upon arrival, prisoners are assessed through a Reception Risk Assessment (Wakem \& McGee, 2012: 97). In order to be labelled 'at-risk', individuals must satisfy at least three sections of a long checklist ${ }^{24}$, a process largely reliant on self-disclosure. Once entered in the prison system, a prisoner's 'at-risk' status is reviewed frequently, either through the observation of prison officers, who may see changes to behaviour or mood, or following classification changes that may affect a prisoner's personal circumstances. For example, a prisoner's 'at-risk' status is expected to be reviewed: when they return from court; when any further charges are laid or their status changes; upon entering another prison (after being transferred); if their security classification increases; when they have used force or have self-segregated themselves; if parole has been deferred or if they have been diagnosed with a serious illness. Reviews of a prisoner's 'at-risk' status must also be considered by prison staff if they witness any change in mood or behaviour or if any other knowledge relating to the prisoner prompts concern (Wakem \& McGee, 2012: 98). In order for prisoners to be placed in an ARU, the permission of a prison nurse is required (Stanley, 2011), demonstrating that their use is endorsed at a medical level. ARUs are also staffed by on-site registered nurses with mental health training and visiting forensic nurses and psychiatrists (Stanley, 2011).

Once it has been decided that a prisoner is 'at-risk', they are strip-searched, placed in an ARU cell and subject to frequent observations by officers (Department of Corrections, n.d). Prisoners can be put in ARUs voluntarily or against their will. ARU cells require prisoners to be stripped of all regular clothing (including underwear), and cells are free of any bedding or other loose materials, in efforts to prevent the occurrence of attempted suicides. Prisoners are given 'anti-suicide gowns' to wear, which are made of rip-resistant fabric (National Health Committee, 2010). To reduce the risk of self-harm, prisoners are not given cutlery and are not allowed to keep any personal possessions

\footnotetext{
24 The checklist asks questions such as, 'are you thinking about harming yourself?' and is based primarily around a prisoner's mental health history (Department of Corrections, n.d.).
} 
(Department of Corrections, n.d). ARUs also contain mechanical restraints, such as 'tiedown' beds, which are used to physically restrain prisoners who are at 'extreme' risk of self-harm. While the tie-down bed is reportedly used infrequently, there is a widely held view that such practice is highly detrimental to well-being (Bersot \& Arrigo, 2011).

After being moved to an ARU cell, prisoners are locked in their cells for up to 23 hours a day. They are removed from their cells to shower and are allowed one hour of daily exercise in larger day-rooms, if facilities and staffing allow (National Health Committee, 2010). ARU cells have CCTV cameras and, alongside frequent observations, prisoners are subject to constant light and noise - even throughout the night, where artificial lights are switched on at regular intervals for surveillance purposes (National Health Committee, 2010). The cells provide limited access to fresh air or the outside world, minimal stimulus and sporadic human contact. In many Units, there is no access to television and books (Roguski \& Chauvel, 2009). However, ARU cells are equipped with personal radios and call button intercoms for prisoners to use to communicate to staff.

\section{Legal and policy framework}

In New Zealand, prisoners are entitled to a 'standard of health care [including mental health care] that is reasonably equivalent to the standard of health care available to the public' (Corrections Act, 2004: Section 75). The responsibility for prisoner healthcare, including mental health care, is collectively managed by the Department of Corrections, the Ministry of Health and District Health Boards' Regional Forensic Psychiatry Services (Office of the Auditor-General, 2008). This joint responsibility is evident within ARUs nationally, where custodial, nursing and forensic staff are expected to work collaboratively on the floor to manage and administer care (Department of Corrections, 2012c: M.05.01). Further, prisoners with complex health needs, such as those with severe behavioural problems or disability, including psychiatric illness, intellectual disability, personality disorder or complex medical conditions, are managed in partnership with the forensic team and may be placed in ARUs on a short-term basis (Stanley, 2011). Prisoners with severe mental health needs are managed externally by Regional Forensic Mental Health Services (Office of the Auditor-General, 2008). 
The Department's national operating policy ${ }^{25}$ (Department of Corrections, 2012c: M.05) outlines the official response and expectations around identifying, observing and managing 'at-risk' prisoners. The Corrections Act 2004 (s 60(1)(b)) allows prison managers to segregate prisoners in order to 'assess or ensure a prisoner's mental health (including, without limitation, the risk of self-harm.)'. While segregated, prisoners deemed to be 'at-risk' must be visited by health professionals at least twice a day (s 60(5)(b)). The Prison Service Operations Manual (PSOM) provides operational guidance for ARU staff when assessing and managing prisoners 'at risk of self-harm' (Department of Corrections, 2012c: M.05.04). The PSOM is largely framed around risk assessment and oversight obligations, providing staff with direction surrounding assessment, reassessment, recording and monitoring of prisoners in ARU cells. The PSOM also highlights the roles and accountabilities of inter-disciplinary staff members working within the Unit (Department of Corrections, 2012c: M.05.04). Despite 'at-risk' prisoners being wholly excluded from the mainstream regime, the PSOM stipulates that 'at-risk' prisoners should 'have the same opportunities for involvement in prison activities as other prisoners, consistent with maintaining their safety and the safety of others' (Department of Corrections, 2012c: M.05.03.01).

\section{Strategic approach to management of self-harm and suicide risk}

Some obvious strides in policy development have been recently taken by the Department to rehabilitate prisoners and to reduce recidivism. In the wider field of health and mental wellbeing, initiatives include the introduction of the High Dependency Unit (HDU) in RP and the roll out of dedicated Drug Treatment Units (DTUs). The 20-bed HDU was jointly set up in 2012 by the local DHB, Ministry of Health and Department of Corrections in order to provide around the clock care to ageing prisoners with significant, often age-related, health needs. The HDU is ground-breaking in that it is run by health staff and supported by a custodial team, which emphasises ageing with dignity and the provision of care as central governing principles (State Services Commission, 2013). The first DTU was introduced in 1996 and is modelled as a 'therapeutic community', taking a group-based approach to rehabilitation, based on a 'culture of respect, collaboration, openness, responsibility, and support' among prisoners and staff (Controller \& Auditor General, 2012: 3.28). Both initiatives

25 Titled 'Prisoners at risk of self-harm'. 
represent a shift away from traditional penal discourses of control and management, at a strategic, official level (Garland 2001; Controller \& Auditor General, 2013; Department of Corrections, 2014b).

Despite these isolated examples, New Zealand's response to 'at-risk' prisoners remains highly managerialistic in approach. In terms of mental health care, the increasing number of external and internal health care staff to provide care has been a positive development. However, actuarial risk assessment tools still present the primary means to identify and triage different categories and classifications of mental health, self-harm and suicide risk, which can inadvertently undermine a more 'inclusive and therapeutic' response to prisoners' issues (O'Malley, 2004: 326). Similarly, developments in policy relating to 'at-risk' prisoners, such as the introduction of ARUs, rip-proof bedding and razor blade restrictions, represent a preventative and custodial response to prisoner distress, given their preventative rather than recovery-based approach. Such approaches are characteristic of 'the new penology', a set of penal discourses centred on the minimisation of risk that prioritises the control of internal systems over the rehabilitative needs of individuals: 'the task is managerial, not transformative' (Feeley \& Simon, 1992: 452). An on-site review of ARUs in 2008 suggested that ARUs needed to become more caring and therapeutic environments, if they were to be aligned with other jurisdictions. This led to some minor changes in operational policy, such as the introduction of a new admission risk assessment process ${ }^{26}$. Despite these developments, the next section highlights how ARUs (as a central element of at-risk policy) have changed little since their inception, remaining limited in their ability to provide care or mental rehabilitation to prisoners.

\section{Commentary and issues raised in New Zealand}

While New Zealand's ARUs have not yet been the subject of research in their own right, they have been discussed in other research and commentary. As part of this wider examination of practice, many parties have expressed concern regarding both the use and misuse of ARUs (Human Rights Commission, 2009; Roguski \& Chauvel, 2009; Stanley, 2011; Wakem \& McGee, 2012). The National Health Committee (2010: 57) suggested a full review of ARUs, with particular focus on how to limit seclusion and how

${ }^{26}$ Information received from staff member at HB Prison (23 February, 2015). 
to appropriately manage other 'types of risk', for example drug withdrawal or behavioural issues. Watchdog agencies, such as the Ombudsman (Wakem \& McGee, 2012), expressed concern over the isolating nature of ARUs. They recommended that the units should only be used for short-term oversight, as a matter of last resort and for a select few prisoners (Wakem \& McGee, 2012). Prisoners held in ARUs have also described them as 'a form of extra-judicial punishment' (Toia $v$ Department of Corrections [2014], at [13]). Alternatively, certain prisoners have regarded them as damaging spaces:

The worst thing you could do to me is to put [someone in] At Risk ... time drags, nothing to do... to sit in a cell day-after-day with nothing to do it just drives me insane... they say it's for your safety but it's actually just degrading (cited in Roguski \& Chauvel, 2009:12-13).

Prisoners have also spoken of the degrading nature and impact of being forced to wear suicide-proof gowns with no underwear (Roguski \& Chauvel, 2009). In order to avoid placement in an ARU, prisoners have been known to self-regulate emotional behaviour and dissuade disclosing difficulties to staff, which can leave mental health issues unchecked and unaddressed (Roguski \& Chauvel, 2009; Indig et al., 2010 cited in National Health Committee, 2010). Prisoners spoke of managing and regulating their emotional distress through other channels, for example via supportive interactions with the prison chaplain or through self-medication with illegal drugs (Roguski \& Chauvel, 2009).

Commentators are also concerned that ARUs are overused. While official policy establishes that ARUs should only be used to 'manage and observe' prisoners with active self-harming or suicidal ideation, in reality the units manage a much wider range of prisoner behaviour. Due to a lack of alternative units, ARUs are used for 'behaviour management, detoxification and 'time out' or punishment' for other vulnerable prisoners (National Health Committee, 2010: 35). The Ombudsman noted that it is particularly common for volatile prisoners who show signs of 'highly disruptive and disturbed behaviour' to be physically contained and managed in ARUs (Wakem \& McGee, 2012). Prisoners with lower level issues can also be informally diagnosed as 'atrisk' when showing bursts of emotion (National Health Committee, 2010). Official guidelines dictate that prisoners with acute mental health needs should be outsourced to an external Regional Forensic Psychiatry Service (RFPS) for treatment (Wakem \& 
McGee, 2012: 93; Department of Corrections, 2012c). However, prisoners with acute mental health problems are reportedly contained in ARUs, which are used as a type of holding pen, until more appropriate external care can be made available (Office of the Auditor-General, 2008). The Department of Corrections confirmed via an Official Information Act (OIA) request that there were over 3,000 prisoner placements in New Zealand ARUs in 2011 alone (Brooking, 2013).

Both the Ombudsman's Office (Wakem \& McGee, 2012) and the Human Rights Commission (2009) have expressed concern regarding the lengths of stay in ARUs. Despite the units being designed to only house prisoners for short-stays (up to one week), prisoners have been kept in these units for months (Human Rights Commission, 2009), with one prisoner held for 18 months (National Health Committee, 2010). One prisoner recounted his experience of being placed in an ARU following his initial upset at being separated from his children. A night's initial observation in the Unit turned into more than three weeks; at this point, he 'was going nuts' (Roguski \& Chauvel, 2009: 13). This example demonstrates how ad hoc placement in an ARU can be.

So far, the chapter has 'set the scene' in terms of summarising New Zealand's current legal, policy and operational framework surrounding the use of ARUs, as well as presenting some of the criticisms that have been raised in relation to their practice, including from the perspective of 'at-risk' prisoners. While commentators in New Zealand recognise that ARUs serve a useful purpose - to keep prisoners physically safe in extreme circumstances and for short periods of time - issues have been raised, in particular, around their overuse, misuse and lack of oversight. The next part of this chapter will introduce some initial research findings, which confirm that ARUs are currently being used to contain and manage multiple behaviours and a wide range of risk factors. The findings also provide some useful context in relation to the overuse and misuse of ARUs, from the perspective of staff, who often feel limited in their ability to provide other placement options for less 'legitimate', or severely 'at-risk', prisoners. 


\section{The use of ARUs}

This research included a three month ${ }^{27}$ 'snapshot' of prisoner files and staff notes relating to daily ARU practice across two sites. This data, and subsequent interviews, found that ARUs were frequently overused and misused. While no deaths occurred over this three-month period, over 150 prisoners were placed in ARUs across both sites, with many prisoners facing repeated or lengthy stints. Notes accessed from IOMS highlighted that prisoners were being held in ARUs for a wide range of purposes and exhibited diverse needs or 'risk' factors, beyond risk to self. There was also a widely held view among interviewed staff that prisoners were deliberately and manipulatively placing themselves in ARUs, in order to escape elements of the wider prison environment - a practice that hadn't been raised in previous commentary or reports.

Table 4 shows that there were 81 admissions (comprising 63 prisoners) to the RP ARU and 109 admissions (comprising 94 prisoners) to the HBRP ARU in the period between 1 June and 31 August 2014. The higher number of ARU placements than number of prisoners indicates that some prisoners experienced multiple stints in the ARU. Some prisoners bounced back and forth to the ARU multiple times in the 3-month period. In several instances, prisoners were housed in the ARU for the entire period, despite the ARU being designed for stays of up to one week (Roguski \& Chauvel, 2009).

27 Over the period of 1 June - 31 August 2014. 
Table 4: Number of prisoners and placements in RP and HBRP's ARUs, 1 June -31 August 2014

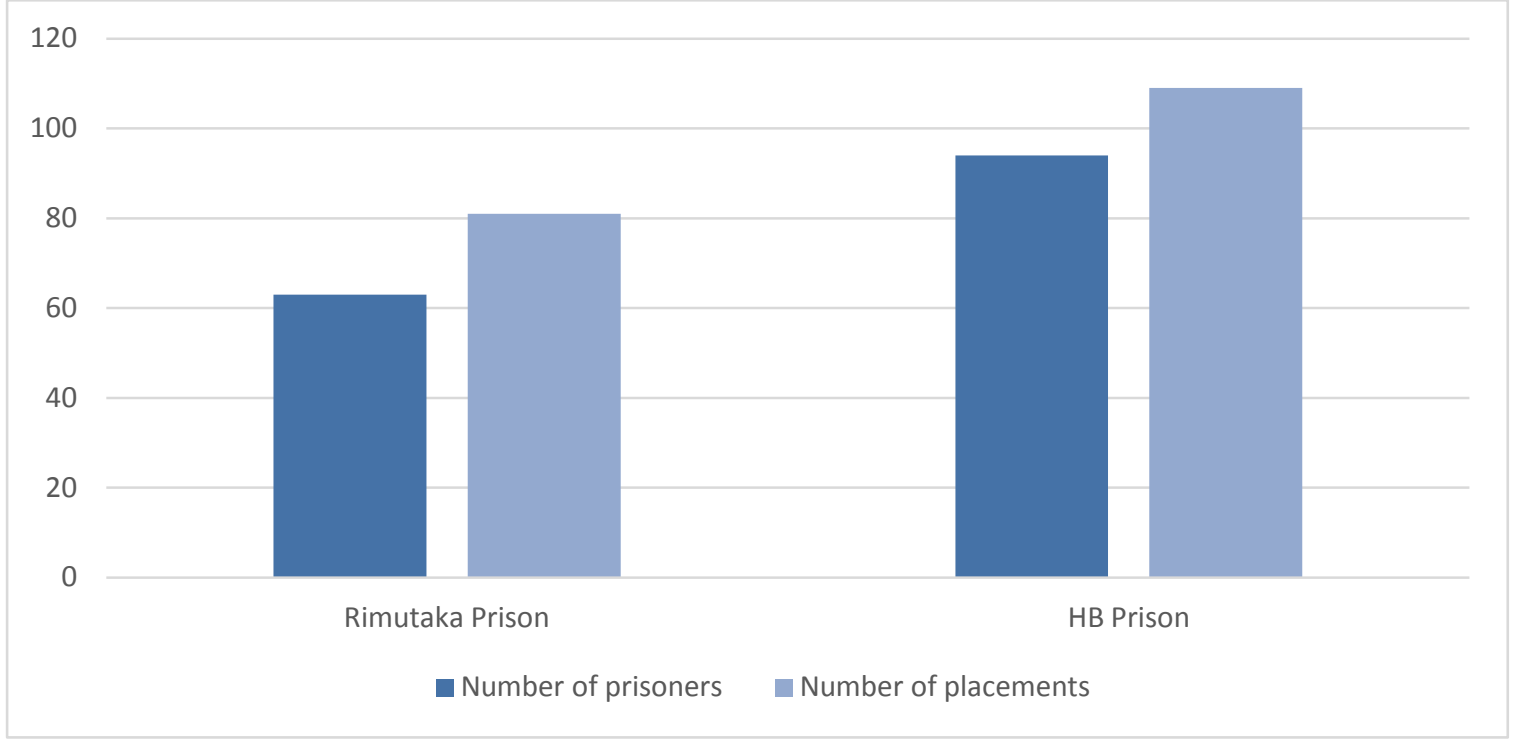

Table 5 is drawn from a range of prisoner files and staff notes relating to 153 prisoner admissions across both RP and HBRP, during the three month 'snapshot'. The data indicates 'reporting counts', rather than numbers of people affected or admissions. There are more 'reporting counts' than number of admissions, as when prisoners have been held in the ARU for multiple reasons, all reasons are accounted for in the table. As such, there are more 'reporting counts' than actual prisoners. While there were over 190 admissions across the two ARUs over the three month period, in relation to HBRP specifically, a number of prisoner file notes relating to ARU placement provided either unclear or unrecorded reasons for admission, or were totally inaccessible. In light of these factors, the table overleaf is not entirely comprehensive; similarly, the reporting counts from the two prisons are not directly comparable. However, the data still provides some useful context when considering how and why ARUs are used on a regular basis. 
Table 5: Reasons for admission to ARU in RP and HBRP, 1 June - 31 August 2014

\begin{tabular}{|c|c|c|c|}
\hline REASON FOR ADMISSION TO ARU & $\begin{array}{l}\text { Reporting count } \\
\qquad R P^{28}\end{array}$ & $\begin{array}{c}\text { Reporting } \\
\text { count } \\
H B R P^{29}\end{array}$ & $\begin{array}{l}\text { TOTAL } \\
\text { Reporting } \\
\text { count }\end{array}$ \\
\hline Physical health & 5 & 4 & 9 \\
\hline $\begin{array}{l}\text { Non-medical oversight: disease and } \\
\text { illness }\end{array}$ & 2 & 0 & 2 \\
\hline ○ Non-medical oversight: injuries & 1 & 3 & 4 \\
\hline$\circ$ Medical oversight & 2 & 1 & 3 \\
\hline Self-harm and suicide attempts & 33 & 14 & 47 \\
\hline ○ History of self-harm & 8 & 3 & 11 \\
\hline$\circ$ Indicated thoughts of self-harm to staff & 13 & 9 & 22 \\
\hline $\begin{array}{l}\text { Indicated thoughts of self-harm to } \\
\text { others }\end{array}$ & 3 & 1 & 4 \\
\hline$\circ \quad$ Active/recent self-harming & 6 & 1 & 7 \\
\hline$\circ$ Recent suicide attempt & 3 & 0 & 3 \\
\hline First time prisoner & 3 & 8 & 11 \\
\hline 'Placement issue' & 3 & $\mathbf{0}$ & 3 \\
\hline$\circ$ Short stay prisoner & 2 & 0 & 2 \\
\hline$\circ \quad$ No beds in other units & 1 & 0 & 1 \\
\hline Detoxification/withdrawal from AOD & 5 & 1 & 6 \\
\hline Mental health & 14 & 8 & 22 \\
\hline $\begin{array}{lll}\circ & \text { Exhibiting } & \text { symptoms } \\
\text { hallucinations, hearing voices) }\end{array}$ & 5 & 3 & 8 \\
\hline Mental health history & 1 & 2 & 3 \\
\hline Forensic patient & 5 & 3 & 8 \\
\hline Reception from secure forensic facility & 3 & 0 & 3 \\
\hline $\begin{array}{l}\text { Not coping in mainstream prison } \\
\text { environment/safety fears }\end{array}$ & 5 & 11 & 16 \\
\hline $\begin{array}{l}\text { Distressed, emotional or anxious } \\
\text { behaviour }\end{array}$ & 8 & 15 & 23 \\
\hline Recent death in family & 1 & 1 & 2 \\
\hline Behavioural issues & 10 & 17 & 27 \\
\hline$\circ \quad$ Non-compliance with officers & 2 & 5 & 7 \\
\hline o Violent/volatile behaviour & 4 & 10 & 14 \\
\hline$\circ$ Unclear & 4 & 2 & 6 \\
\hline Processing issue & $\mathbf{1}$ & $\mathbf{0}$ & 1 \\
\hline Non-English speaking & 0 & 1 & 1 \\
\hline Unclear/unrecorded & 5 & 14 & 19 \\
\hline TOTAL & 93 & 94 & 187 \\
\hline
\end{tabular}

ARUs as a 'dumping ground'

Table 5 highlights how ARUs are currently used to manage: prisoners with mental health concerns, including prisoners experiencing hallucinations and those with personality disorders; prisoners experiencing detoxification from drugs or alcohol;

28 Drawn from 81 ARU admission notes in total.

29 Drawn from 72 ARU admission notes in total. 
violent or non-compliant prisoners - a particularly common reoccurrence; deportees and non-English speaking prisoners, whose levels of risk could not be easily ascertained by staff; prisoners concealing drugs internally; and prisoners expressing fears relating to their personal safety, for example those being stood over for medication or food in the mainstream. ARUs are also used: to separate gang members; to house 'vulnerable' prisoners, such as youth or first-time prisoners; and to contain prisoners who are recently transferred from other prisons. Prisoners with physical health issues, ranging from fractures to heart conditions to infectious diseases, are also placed in the ARU under either medical oversight (Corrections Act 2004: section 60) or more informally for the purposes of safety. In summary, while 'at-risk' policy is designed somewhat narrowly to respond to and manage self-harm risk, the ARU serves a much broader range of functions in practice.

Extremely high-needs prisoners are contained in ARUs, which can be used as a waiting room until appropriate care or management is organised, such as admission to Kohanga Reo-Purehurehu ${ }^{30}$ (Regional Forensic Secure Unit). While many prisoners in these units cited more 'legitimate' suicide or self-harm related concerns, such as recent suicide attempts or visible emotional distress, the files indicate that many prisoners strongly and repeatedly denied thoughts of suicide or self-harm to staff. As mentioned, no clear definition for the term 'at-risk' presently exists; rather, a prisoner needs to satisfy the requirements of a check-list in order to be positively classified (Department of Corrections, n.d.). The 'net-widening' of both ARUs' purposes in practice demonstrates that staff perceptions and cultures can feed into formal measurements and assessment of risk, with prisoners being labelled on the basis of 'subjective judgements' and wider institutional demands for security management and control, rather than immediate or deliberate risk of self-harm (Hannah Moffat, 1999: 81). As one interview participant put it: 'This is a dumping ground for when you can't find a place for somebody' (Nurse, HBRP). Other ARU staff members described ARUs as 'the ambulance at the bottom of the cliff' and 'the end of the line' for 'at-risk' prisoners, who generally are viewed to have no other placement options available to them within the wider prison (CO, HBRP; CO, $\mathrm{RP})$.

${ }^{30}$ Referred to commonly by staff, and throughout this thesis, simply as 'Purehurehu'. 
Interviews with staff members across both ARU sites uncovered why prisoners tend to 'drift in and out of ARUs' in such a frequent and informal manner (Wakem \& McGee, 2012: 101). Many prisoners were placed in the ARU due to 'placement issues', in which prisoners with diverse issues could not be guaranteed safety in any other part of the prison. In some circumstances, for example when the prison was experiencing 'muster blowout', staff reported that there were simply no other beds for prisoners, regardless of their 'at-risk' status. In a similar vein, while older prisoners with specialist health needs in RP can be held in their HDU, staff from HBRP noted how their ARU at times ran 'like a geriatric ward' (CCDHB worker, HBRP). In HBRP in particular, volatile and disruptive prisoners were often placed in the ARU for behavioural management; the ARU, and sometimes the use of mechanical restraint, were used as a way to control unruly or volatile prisoners. As noted previously, ARUs should be used as a matter of last resort and only in the most extreme circumstances to protect a prisoner's safety.

A key 'placement issue' relates to the care of mentally unwell prisoners. Many staff members viewed mentally unwell prisoners as inherently 'at-risk'; however, some interdisciplinary conflict existed between custodial and health care staff in relation to the treatment of mentally unwell prisoners who weren't at direct risk of self-harm. Despite the heightened negative impact that segregation can have on mentally unwell individuals (Morgan et al., 2007), health staff were unified in the view that non selfharming mentally unwell prisoners should still be placed in ARUs due to their inherent vulnerability. One worker highlighted this tension, by noting how:

[The Corrections officers] go back to the Corrections mandate which is 'an ARU is for people who are at-risk of killing themselves'. Well that's the at-risk mandate, whereas as a mental health practitioner, there is nowhere else in the prison for those offenders who are mentally unwell, who by virtue of their mental illness will put themselves in the path of risk of others, whether it be because they're manic and they're over-intrusive, whether it be because they've got no impulse control, whether it's because they're completely vulnerable to what's going on around them (CCDHB worker, RP).

Health staff also acknowledged the lack of safe alternatives in the wider prison environment, when responding to the needs of many mentally unwell prisoners: 
There's one or two officers, particularly, who are in the ARU at the moment who are very, very loudly saying 'we are not a mental health holding unit' - yeah you kind of are. You kind of are the prison mental health unit, because we don't have anywhere else. We don't have anywhere else that is safe, that is monitored with staff that are educated, who can give the same calibre of care as you give. So, you are our default position (CCDHB worker, RP).

We even get custodial saying 'this person isn't at-risk, they don't want to selfharm' but they are actually at-risk because they are so mentally unwell that they couldn't survive down in a unit - that does put them as at-risk. I think we need to change the definition of the ARU (Nurse, RP).

In more extreme circumstances, where prisoners required externalised care, the ARU was described by one CCDHB worker as 'a waiting room for the hospital', a necessary measure in the short-term.

Some Corrections officers thought that placement of mentally unwell prisoners in the ARU was beneficial to the extent that it allowed for heightened access to forensic oversight and services. Simultaneously, removing mentally unwell prisoners from the mainstream alleviated stress and pressure on correctional staff, who often felt poorly equipped to respond to such issues. One custodial officer noted:

Unless you have a place where you can move those people from, from the main jail area or a bed unit because someone's hearing voices, to this area here where they can get proper supervised treatment, you're going to be in all kinds of trouble (CO, HBRP).

Many staff similarly commented on the lack of safe alternatives for a broader cohort of 'vulnerable' prisoners, who were unable to cope with mainstream prison life. Officially, prisoners struggling in the mainstream environment have the option of being placed in 'voluntary protective custody' (Department of Corrections, 2012c: M.01.05). However, staff noted that the process to become voluntarily segregated had becoming increasingly fraught, leading to rampant overuse of the ARU. One CO explained the shift in practice:

A few years ago they made voluntary segregation harder to get, previously it used to be 'I fear for my safety, sign here, you're on segs'. But now there's a process and a rigmarole and it's almost put a vol segs application into the too hard basket, so the new default is to put people at-risk.... I think they made vol segs harder to get as a reaction to it being too easy, now we're feeling the backlash in here (CO, HBRP). 
In line with many male prisons (Crewe et al., 2014), staff from both sites noted that mainstream units were dominated with macho cultures of hierarchy, mistrust, fear and aggression. Gang culture and stand over tactics, such as blackmail for 'rent', were also rife (National Health Committee, 2010: 32). One nurse noted:

Some of them just want time out, some peace and quiet. The prison environment it's hard out there for them. There's a lot of expectations for them to conform to the way of the units, a lot of them come here for a break from that. Some of them are getting stood over for their nicotine patches, or their medications if they're on tradable medications... So some of them will say they've got thoughts of self-harm to get away from that, [if] they're not getting their medication [they] starting to feel on edge, anxious. I see it as they are more scared out in the unit, or being bullied, that kind of thing (Nurse, HBRP).

However, few staff shared such an empathic view of prisoners and their needs. Many staff spoke of such prisoners in a negative light, describing them as 'manipulators' and 'malingerers', who they 'had to put up with'. Many officers blamed the prisoners themselves for 'crowding' the ARUs:

All these hangers-on, the fraidy cats who want to come here because it's the easy option, that's sort of almost a sub-aim if you like, which is being forced on us by the prisoners themselves. They see this is the easy option (CO, HBRP).

Despite the perception that high number of prisoners deliberately manipulated their placement, staff were reluctant to remove any prisoners out of the ARU who vocalised thoughts of self-harm. As a result, staff noted how prisoners who 'feigned' self-harm remained in the ARU for lengthy periods of time, particularly in the HBRP ARU. One staff member spoke with frustration about one prisoner who they perceived as intent on spending his entire sentence within the ARU, by continuously expressing thoughts of self-harm:

They know what to say a lot of the time, pretty much. I'm not even $100 \%$ convinced that anyone of these in here now should be here. But these guys learn to say the right things, it keeps them here. There's another one who says 'ohh I'm gonna stay here until I get out, coupla weeks'. But he's fine (Nurse, HBRP).

The risk averse culture of the Unit prompted a sense of 'self-protection' among some staff. The influence of risk averse cultures in decision-making was directly linked to the overuse of the ARU, particularly in HBRP. As one officer noted: 
I always err on the side of caution because I've learnt that you're better to be safe than sorry. So if you're keeping a guy in there another 24, 48 hours, it doesn't matter. You've got to always err on the side of caution (CO, HBRP).

The use of ARUs to house 'non-legitimate' prisoners has important implications for practice and interpretations of risk, as discussed below.

As with many prison environments, burnout, fatigue and stress was common among those working with 'difficult' prisoners (Liebling et al., 2011). Despite the ARU presenting numerous challenges, staff highlighted how the ARU was also viewed as a useful tool for staff working outside of the ARU, in that it gave them 'time out' from disruptive or violent prisoners. In HBRP in particular, behavioural outbursts in the mainstream prison environment were responded to by management and control, such as placement in the ARU, rather than via meaningful engagement and intervention (Liebling et al., 2011). Within HBRP, staff reportedly manipulated placements as a way to 'have a break' from the high-needs prisoners, who they did not feel adequately trained to respond to:

It's easier to keep people who are not at-risk in an ARU. So you're not doing the obs[ervations], you're not doing finger food, you don't have to observe them during showers. So it's bums on beds but it's no paperwork really, it is easier. They like filling it up with the non-at-risk stuff. That's my impression... That's why they always want us to clear the at-risk prisoners before they would ever move a nonat-risk prisoner (Nurse, HBRP).

Further, staff in HBRP likened the ARU's purpose to that of a high security Management Unit; the non-existence of such a specialist environment in HBRP, and the existence of a Management Unit ${ }^{31}$ in RP, may account for some of the differences in practice across the two sites.

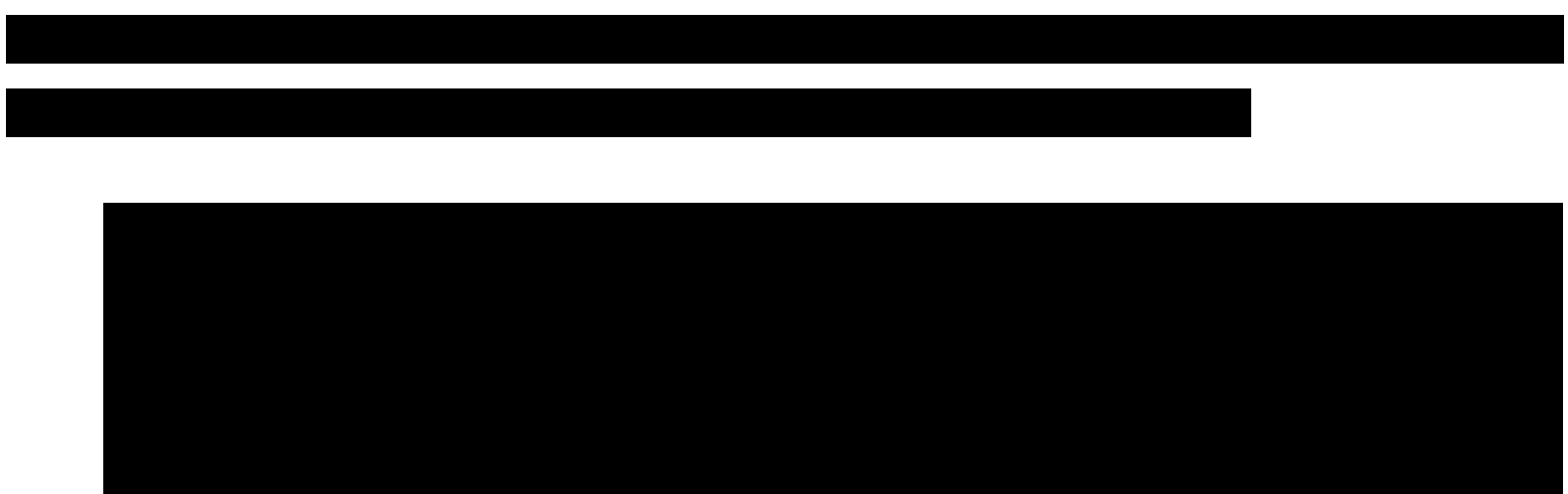
31 Management Units are analogous purpose-built solitary cells, which are used for prisoners who exhibit
violent or disruptive behaviour. 


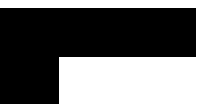

The ARU was also used for prisoners in transit to other prisons. Such an approach was favoured by custodial staff as it required less paperwork than placement in the mainstream would require, given the ARU's short-stay purpose. One nurse, in relation to another example, noted:

If they've got a court case coming or going to court [the Corrections Officers] like to keep them until court and say to us 'ohh can you clear them when they come back from court?' (Nurse, HBRP).

These examples highlight how the ARU is viewed as an 'easy option' from the perspective of some staff, when managing the placement of high-needs or short-term prisoners. In the HBRP in particular, it seems as though many staff have lost sight of the original purpose of the ARU, directly leading to overcrowded and ambiguous practice on the floor.

\section{Summary}

The Department has made some positive advances in approaching prisoners with mental health issues. However, it appears that recent academic and monitoring reports, as well as Coroner's reports, have indicated several concerns about the use of ARUs, many of which have been confirmed and further expanded on via initial research findings. The issues raised in this chapter identify a number of concerns regarding the legitimate use of ARUs in New Zealand. First, ARUs have a broad function in practice and are used to contain prisoners with a wide range of issues, including some with extreme or severe behaviour which staff are not adequately equipped to deal with. In HBRP in particular, the overuse of ARUs fosters a negative culture among staff who view prisoners as 'blagging the system' to suit their needs. Second, the emphasis on risk management, specifically the avoidance of blame or public fallout following a death or incident in custody, directly leads to crowding within the ARU. Finally, such practice can lead to burnout among staff, who then 'fudge' the administration of policy, for reasons of convenience or to relieve pressures on the floor. The overuse of ARUs exacerbates risk of stress for staff, who are not placed to deal with prisoners with severe or serious needs, such as mental health concerns, and this inadvertently leads to their overuse, in a somewhat cyclical fashion. These exogenous factors are often out of the control of the 
ARU staff working directly within the Units but they have direct implications on the overuse and misuse of these ARUs. 


\section{Chapter Five: 'Risk from Above' and Organisational Risk Management in ARUs}

Risks only suggest what should not be done, not what should be done... To the extent that risks become the all-embracing background for perceiving the world, the alarm they provoke creates an atmosphere of powerlessness and paralysis... [Risk] dims the horizon (Beck, 1997: 141).

\section{The rise of risk}

Since the late 20th century, the governance of public and private sectors has been increasingly guided by risk-based approaches and processes (Beck, 1997; Hood et al., 2004). Risk has become 'the new lens through which to view the world' (Whitty, 2011: 125). Within criminal justice, a growing number of predictive technologies and risk assessment tools have emerged as a means to prevent crime and to categorise and manage behaviour. Risk techniques, replacing a discourse previously grounded in rehabilitation or reform, have been viewed by government agencies as a means to reduce the cost of crime, minimise victimisation and to provide services to those 'atrisk' of reoffending (O'Malley, 2010). Despite the widespread public acceptance of this shift in management, 'debates have raged' about the characteristics of risk management policies which may run counter to, and at times even subvert, 'reform-centred, inclusive and therapeutic' approaches to practice (Bullock, 2011: 120; 0'Malley, 2004: 325). Commentators argue that, within this paradigm, offenders have become defined as 'bundles of harmful behaviours and potentialities' (O'Malley, 2010: 1). In short, the individual needs of those swept up in the criminal justice system have become increasingly reframed as risk factors or 'risky' behaviours that ought to be controlled rather than meaningfully responded to (Hannah-Moffat, 1999).

In the context of prisons in particular, risk management has become 'a key organising principle of contemporary correctional practice and offender management' (Maurutto \& Hannah-Moffat, 2006: 438; Feeley \& Simon, 1992). As a means of ensuring efficiency, cost-effectiveness, good order and the 'avoidance of failure', prisoners have become increasingly classified by levels of assessed dangerousness, via the use of risk assessment tools and other actuarial techniques (Whitty, 2011: 126). In doing so, 
'individualised diagnosis and response is displaced by aggregate classification systems for purposes of surveillance, confinement and control' (Feeley \& Simon, 1992: 452). Similarly, there has been an increasing institutional emphasis given to the way in which the prison system mitigates and manages its own 'organisational risk', such as the financial or legal fallouts which can follow publicised incidents or damning 'prison scandals' - for example, violent riots or preventable suicides (Black, 2005; Whitty, 2011: 124). While much of the literature considering penal risk management has focused on the control of violent or dangerous offenders, risk management discourses also permeate the daily routines and lived experiences of 'at-risk' and other vulnerable prisoners, across jurisdictions (Liebling \& Arnold, 2005; Cliquennois \& Champetier, 2012; Ricciardelli et al., 2015).

While critical commentators often view risk-centric approaches to be inherently problematic, others have highlighted its diversity of form and its ability to provide a positive platform for services and protections, such as programmes or treatment for both 'at-risk' and 'risky' populations (O'Malley, 2004; Whitty, 2011). Further, risk is not a 'unitary or monolithic technology' (Seddon, 2008: 302). Despite remaining an underresearched area, commentators have noted the significant impact that various cultures and contexts can have in terms of the way risk is 'conceived, understood, manipulated and managed' on-the-ground (Ericson, 2007: 965).

Bearing these considerations in mind, this chapter will unpack the way in which 'organisational' or institutionally prioritised risk management is perceived by staff, within the ARU setting. These findings will highlight the degree to which risk is prioritised and the way it influences or inhibits the care of 'at-risk' prisoners. Not only has there been little research considering how, or the extent to which, institutional risk management priorities impact on everyday prison practice, there is also a limited understanding of how these strategies are used to manage primarily 'at-risk', as opposed to dangerous or 'risky' individuals (Murphy \& Whitty, 2007; Ricciardelli et al., 2015). Similarly, little research has considered the varying ways in which 'risk' is interpreted by staff from different disciplinary backgrounds, and whether these interpretations converge to provide positive platforms or whether they give rise to barriers for staff who work within such a 'high-risk' part of the prison environment. In sum, the practical working dynamics present an intriguing forum in which to consider 
the influence, manifestations and interpretations of risk when staff care and respond to the diverse range of prisoner needs present within the ARUs.

\section{The working dynamics of 'organisational risk'}

As noted above, reducing suicide and self-harm in many prisons is primarily underpinned via a risk management approach which, in practice, prioritises the prevention of physical harm over the amelioration of mental wellbeing. When considering the approach to suicide prevention in prison, a focus on risk management 'from above' is particularly acute, given the high levels of public scrutiny and, in some jurisdictions, potential legal fallout following suicides under state care (Whitty, 2011). The institutional focus on managing and alleviating risk, and its impact on staff attitudes and day-to-day practice, were recurring themes throughout the fieldwork process across both sites - perhaps unsurprisingly, given the focus and title of the environment in question. On the ground, staff were conscious of the impact of 'organisational risk' (Whitty, 2011: 124) and its influence in shaping the current approach to 'at-risk' prisoners in both HB and RPs:

The whole point from Corrections' point of view is to stop people self-harming. If the call has to be made to keep people safe or take a small risk to help them get better, Corrections would rather have someone unwell and in a situation where they're not able to self-harm than put them in an environment that would perhaps give them the opportunity to self-harm but would be more beneficial for them to get better. Because of the duty of care and having to answer questions and things like that. Just to avoid the embarrassment to the Department (CO, RP).

Self-harm, suicide and the prediction of such incidences is a 'blurry' phenomenon, at best. Not only are risk factors relating to suicide and self-harm wide-ranging and diverse, they are concentrated in and complicated further by the prison environment itself (Liebling, 2001a). Staff members across both sites highlighted the uncertain nature of self-harm and suicide risk and, in one case, the luck that sometimes accompanied 'successful' outcomes for self-harming prisoners within the ARU:

You have quite a significant amount of the prison population that will walk in with thoughts of self-harm, due to the situation that they're in. As it is with young, males, the poor impulse control can often be the precipitator to a suicide attempt or an actual success. And so you can have all the checks and balances in place and have done everything that you can around someone and then they have a bad phone call with Mum and they go back to their room and they think 'Fuck it all' 
and that night they hang themselves. And so I think that when you're looking after such a high-risk population anyway, it is difficult, but if you can put all the checks and balances around then the ones that are fairly out there and apparent you can try and be protective of. It's never usually the ones that say 'I'm going to kill myself' that are the ones that you find. And so the situation last Friday ${ }^{32}$ was they went down to get a guy out for a medical appointment - fluke (CCDHB worker, RP).

Staff members highlighted how this uncertainty can rub uncomfortably against the more regimented, actuarial approach adopted by workers when managing and mitigating the occurrence of suicide in prison:

There's an inquiry going on at the moment. Every staff member is interviewed, they get all the paperwork, they go through all the things, they dig fairly deep - did we follow the processes right? What could we have done better? I think inquiries are right if we are falling down or if there is a better method of doing things they identify that and we can put it in place. I don't like to see finger pointing because suicide leaves unanswered questions that you'll never be able to answer. If I looked at the at-risk assessment of that last one, there was never any indication of anything I read that he would commit suicide (CO, HBRP).

[The staff] are worried about the risk that they take if they don't put [prisoners] at-risk and they hang themselves. It's high-risk, you make a mistake like this and it goes to the paper and as much as you don't want to, you do end up taking the can for it. So the officers, because of the culture of the department, the risk averse culture, they don't want to be the ones to say that he's not at-risk and then he hangs himself (Nurse, HBRP).

These accounts highlight the insecurity that managing 'at-risk' prisoners can foster among some staff, as well as the limitations in preventing suicides using a purely risk management approach. Coupling the unpredictable nature of suicide with a risk-averse culture gives rise to unique ambiguities and stresses among staff, who may feel 'caught in the middle' between institutional priorities of 'zero risk' (Walklate \& Mythen, 2011: 107) and the unpredictability of 'at-risk' prisoners, particularly those who are not housed in the ARU. In relation to forensic patients in particular, staff were attuned to the personal ramifications that might occur if risks are not adequately managed; as one staff member noted, 'if they get out into the community and it all goes tits up, we wear it' (CCDHB worker, HBRP). As outlined in the previous chapter, institutionally consuming, 'top down' risk cultures can directly lead staff to act in an overly cautious and security-oriented manner when determining the suitability of a prisoner's placement within the ARU.

\footnotetext{
32 Referring to an attempted hanging incident.
} 


\section{Positive and negative cultures of risk}

So far, this thesis has highlighted the existence of a risk averse, security-centric culture and the impact it can have on staff when determining placement of prisoners in the ARU, to the extent that it causes feelings of hyper-vigilance or cautiousness when assessing prisoners. The rest of this chapter will delve deeper into the dynamics of risk during the everyday practices and routines of staff who work within the ARU, exploring how 'organisational risk' can be perceived and interpreted differently depending on each ARU's specific cultural, disciplinary and organisational contexts. In particular, this section considers how cultures of risk can act to both foster and undermine a more caring, supportive approach to prisoners held in the ARU.

\section{Positive risk cultures}

Risk can take a variety of forms. O'Malley (2004) argues that risk discourses do not have to give rise to exclusionary, polarising practice and that it can, in fact, promote inclusive and unifying responses or risk-needs based services, particularly in prisons. Furthermore, risk assessments can be used as positive tools to facilitate therapeutic interventions for prisoners and to integrate welfare based on rehabilitative approaches 'within the risk agenda' (O’Malley, 2004: 328). Whitty (2011) also views risk-based approaches as positive and in the interests of prisoners, noting that a risk management approach can work to foster compliance to human rights obligations within institutions, in the interests of reputational protection. While interviewees predominantly framed risk as a barrier to humane or care-oriented treatment, some staff spoke of its ability to drive good practice within the ARUs. At RP, in particular, staff cited a 'climate of support rather than punishment' (CCDHB worker, RP). Here, the ARU was frequently used as a short-term holding pen, while staff worked tirelessly and pro-actively to tailor individual responses for each prisoner in the unit. Staff members highlighted how the ground-level 'climate of support' within the ARU provided a positive platform for 'atrisk' prisoners and their outcomes:

When [the prisoners] are hesitant to leave the ARU it's that time that we could go down with [the] PCO and talk to them, as to why they are hesitant - is it the fact that they still have thoughts of harm, or is it the fact that they're just scared to leave the ARU? And then we'll go back and we have that conversation together, dependent on what the patient's answer is... So there's a lot of communication 
between us - taking time, listening not rushing, because you've got other work to do. The whole concept has changed in the ARU (Nurse, RP).

Here, health and correctional staff worked together to ensure that prisoners safely progressed from the ARU into the mainstream. Staff also highlighted the way in which supportive staff cultures could work to shift the focus of risk to individual need, rather than on compliance with institutional demands or official policy, when required. One staff member highlighted this dynamic:

We had a case of a guy who never came up to at-risk and he was highly suicidal, and the reason for not coming up was he said that if he did it would make things worse... So what the unit did, they actually ended up double bunking him with one of his friends, and they've been staying together for a while. It was an intensive management plan put in place, monitoring his well-being and he actually overcame that and never came to the ARU. It's just done, I don't know whether it's in the policy, but it's done and he's safe and he's fine (Nurse, RP).

In this instance, staff prioritised the interests of the individual prisoner over compliance with official policy in a less rigid manner, as a means to successfully manage risk. While staff described RP's ARU as being uncharacteristically full during the time of interviews, participants highlighted how 'at-risk' prisoners benefited from a much higher ratio of staffing and 'connectedness' with ARU staff when compared to the mainstream prison, given the small numbers of prisoners generally housed within the unit.

The culture of support and care at RP was less evident among the discussions of day-today practice among HBRP staff, although they spoke positively of each other. Here, a CCDHB worker highlighted how working together to mitigate risk provided a positive means of strengthening multi-disciplinary interests under a 'common cause':

[Custodial and health staff] priorities converge because of risk. This is the ARU, anyone who comes here is at risk of something. And if the risk is caused by mental illness they are really wanting to treat it because that will reduce the risk and allow the person to get out of here (CCDHB worker, HBRP).

While MDT dynamics will be discussed in more detail in Chapter Six, it useful to note the positive contribution that risk-centric ideologies can have in bringing together conflicting disciplinary interests and priorities. 
While RP's 'climate of support' led to more positive interpretations of risk management, multiple staff members within the HBRP ARU spoke of an on-the-ground culture of rigidity, with 'black and white' adherence to rules guiding routine practice. Such an approach limited the ability for staff to tailor responses to individual need. In order to manage risk within the ARU, rules could be applied inflexibly, even when such an approach resulted in negative outcomes for prisoners. One staff member highlighted this somewhat paradoxical tension:

It's very, very rigid here... It's black and white for Corrections. For example, we had a guy who was having seizures who was being controlled and restrained. So he kept having epileptic seizures and they were controlling and restraining him because he had assaulted someone. So we had to get him over here because he kept having fits, and so I wanted him where we could manage him to see if we were gonna send him out [to hospital]. So they put him in the round room, this guy, and I said 'don't put him in the round room, put him in an ARU cell and put him in ordinary clothes, don't put him in a gown, he's not at-risk of self-harm'. But because it's an ARU they then tried to strip this guy - that made him get angry and have another seizure. He's a guy that when he gets angry he has a seizure. So he had another fit and I sent him out by ambulance in the end. We could have contained him here if they'd left him in his ordinary clothing in the ARU. But they had this blanket thing that if you're in the ARU you have to have the stripped gown... [and] the Department of Corrections lost that in court (Nurse, HBRP).

Staff members from both prisons related how all prisoners were forced to wear the 'atrisk gown', even when they were clearly not at-risk of self-harm, in order to comply with ARU policy. RP staff spoke of occasional instances where low-risk prisoners, such as those who had been 'signed-off' as being 'at-risk' but awaiting placement, were allowed to wear regular prison attire 'for a bit of dignity' (CO, RP). The blanket use of anti-suicide gowns for all ARU prisoners was raised as a negative element of policy multiple times by different staff. One nurse highlighted how such rules and policy were outdated and not always necessary for risk minimisation or prisoner well-being, given that a number of prisoners in the ARU are openly not at-risk of self-harm:

Coz why are they wearing the suicide gown? They're actually not suicidal, but again that relates back to the label of the ARU... the concept or the purpose [of the ARU] needs to be looked at... Things are changing - patients are changing. (Nurse, $\mathrm{RP}$ ) 
With HBRP's ARU often running at maximum capacity, staff were also limited in their ability to treat prisoners' needs distinctly, despite their diversity of needs. Staff highlighted how issues and tensions arose when prisoners were treated differently by staff; as a result, staff took a 'lowest common denominator' approach, treating all prisoners the same despite differing individual circumstances. This often meant the bare minimum was offered to all 'at-risk' prisoners, in the interests of ensuring consistency when managing risk:

Even little things like giving an extra cup of tea, which sounds quite basic, can cause huge issues further down the track if you say you're not going to do that with a prisoner, especially if their mental health is a little fragile as well. We frequently talk about how all of us need to be doing the same thing... Get what you're entitled to and leave it at that basically (CO, HBRP).

Two days ago, a prisoner smashed that phone and it's not being replaced. So anything you bring them is only as good as each prisoner that you give it to. Because you just get one that might lose it. It's very difficult - and it's sad. For some it would be great (CO, HBRP).

Taking such a 'bare minimum' approach is problematic, particularly in very full ARUs, as it suggests that every prisoner is treated as a risk or threat to the security and good order of the unit, despite some prisoners having real and immediate health needs.

While less pervasive than HBRP, staff at RP also spoke of how a blanket adherence to risk-minimising rules and policy could also lead to negative outcomes for 'at-risk' prisoners in a similar way:

I had a guy who had a really low mood. Although he had difficulty concentrating, reading got him out of his own head, but he couldn't take glasses into his cell and he couldn't read without glasses. And so he had 45 minutes of reading during the day and the rest of the time he was left with his own thoughts, and that's really difficult to manage because as a mental health nurse I would be saying 'what can we do to distract you from some of these thoughts, is there something you enjoy watching on TV, is there something that you like reading?' but you're stuck with four walls and a radio... So that's hard, that's frustrating (CCDHB worker, RP).

Anything that would benefit them and stop them being bored would be great, but cards here turn into weapons, they start self-harming. We had a guy who was eating his polystyrene cups, so you know, everything has to be weighed against the risk (CO, RP).

Many staff members are forced to reckon with extreme self-injurious behaviours within the ARU, some of which require a total removal of all items that could possibly be used 
by prisoners to harm themselves. However, these examples highlight how risk management priorities can often supersede individual need and, at times, can work to further undermine a prisoner's mental well-being.

This section highlights how in order for risk to be construed positively, for example by interpreting individual distress as 'needs' rather than 'risks', staff should be adequately resourced and feel supported by others to support and respond meaningfully to 'at-risk' prisoners. While risk can be a useful tool, unifying multi-disciplinary interests under a common banner, its benefits can be compromised when read in light of wider institutional demands, such as reputational protection and blanket adherence to prison rules. Security driven priorities, which may suit the wider prison environment, may not be appropriate or beneficial for prisoners in the context of a specialised unit like the ARU.

\section{Professional dynamics and risk}

A central consideration of this thesis is the extent to which multi-disciplinary staff members can improve outcomes for 'at-risk' prisoners. On-the-ground perceptions and interpretations of risk among distinct organisational groups working in a shared environment is a subject that has received limited research attention. Further, exploring the ways in which healthcare and custody staff perceive and interpret risk provides a useful layer of insight into the 'custody vs. care' dichotomy, which will be explored in more depth in Chapter Six. As such, the professional dynamics of ARU staff, and their potentially distinct perceptions regarding the role and influence of risk within the working environment, is a useful area to explore.

Kemshall (1998) has suggested that a modern focus on risk and containment has reshaped the working practices of professionals, such as nurses or social workers, in the correctional system. Specifically, she suggests that the use of risk technologies has 'rendered [staff] little more than the practitioners of low-grade routines for estimating the risks presented by inmates', further compounding and entrenching exclusionary depictions of risk practice (cited in O'Malley, 2004: 331). On the other hand, O'Malley (2004) suggests that expert practitioners, such as psychiatrists, have confronted and resisted a passive application of actuarial risk regimes. He further suggests that experts have used risk technologies to their advantage, as a means of triaging needs and 
services. Other commentators have noted how distinct organisational 'cultures, practical concerns and contingencies' can influence how risk is applied and interpreted, with risk assessment criteria being applied diversely among those with different professional expertise and backgrounds (Walklate \& Mythen, 2011: 107).

During the course of the fieldwork, it became clear that staff across all disciplines working within the ARU were informed and driven by organisational or institutional risk cultures to some degrees. One CCDHB worker noted:

I think that mental health services and forensic services in particular are pretty risk averse. Corrections are more risk averse in terms of protecting the company than the individual patient, so their focus might be more focus on risk to Corrections than risk to individuals. But that's a concern for everybody as well, it doesn't look good for any service if people kill themselves, that reflects badly on Corrections and it may reflect badly on mental health services as well. I think everybody involved in the business is pretty risk averse - avoiding risk to individuals - to patients, staff and the organisation (CCDHB worker, RP).

While staff from all disciplines were conscious of risk, other CCDHB workers spoke of how the approach could run counter to health-based care approaches, particularly when managing forensic patients:

In the risk assessment model, it's assumed the person won't tell you everything, so you've got to make an assumption. That's what the Health and Disability Commissioner expects of us - that we make an assessment that is more than what the patient has told us. That's not recovery - recovery is collaboratively working with somebody to agree on a pathway forward, and agree on what the problems are (CCDHB worker, HBRP).

This divergence between collaborative, recovery-based approaches to care and presumptive, risk-based approaches to prisoner management was evident in practice. Observations undertaken by Corrections staff, many of whom were greatly admired and respected by forensic and health staff, demonstrated a risk-based and compliance driven approach, rather than a therapeutic or holistic approach, to prisoner care:

I think the nature of the observations is difficult, I think when you have acutely unwell people who are being observed, Corrections officers follow the Corrections mandate. And most of the time they're pretty good but the level of observation is about what they're doing, whereas in mental health the level of observation is how they're talking, how they're presenting, the way that they're presenting things. So for something like sleep, which is so indicative of wellness, you'll have a patient who says 'I'm waking every morning at 3:00 AM and I don't go back to sleep and 
I'm so frustrated and I'm just lying in my bed'. And then you go to the 'obs' and it's like 'slept, slept, slept, slept, slept' or 'appears asleep', which keeps you covered (CCDHB worker, RP).

When comparing custody and health-based approaches to risk, staff detailed divergences in the way 'top down' risk was experienced.

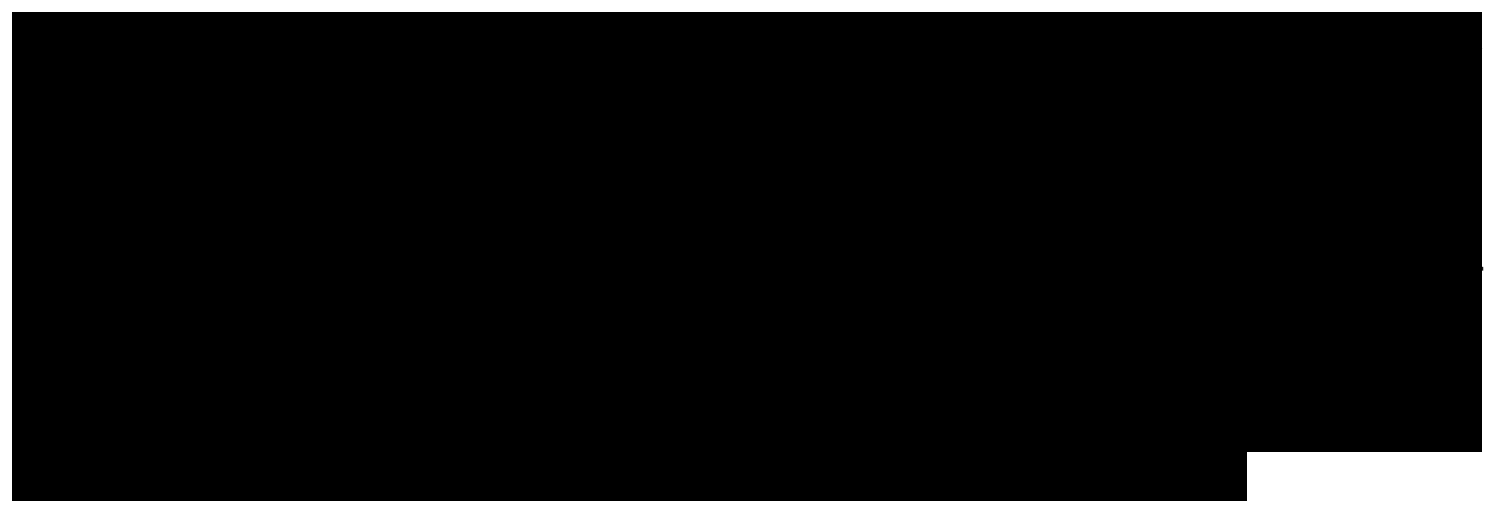

Corrections staff from RP agreed with this viewpoint when describing the sign-off process for 'at-risk' prisoners, suggesting that pressures on forensic staff were not limited to HBRP practice or culture:

From what I've observed it's forensic nurses that are signing off that the prisoner's OK to go down [to a mainstream unit]. It's their professional name I guess you could say, it's their reputation or career even, I guess, to say that that prisoner is mentally sane and can go down (CO, RP).

This point suggests that Corrections officers are also wary of professional risks and may be reluctant to remove prisoners from the ARU when they feel their employment prospects may be on the line. This was perhaps compounded by the fact that, during the fieldwork period, both HBRP and RP had dealt with recent suicides and were particularly alert to the fallout of such risks. One CO from HBRP noted:

... if a prisoner keeps saying he's going to kill himself, do you believe that prisoner is going to do that or you don't? Who are you? I think there is always something of a self-protection or a self-preservation in every human being, that if I do that and he goes out tomorrow and he commits suicide, where do I stand? Do you know what I mean? (CO, HBRP). 
In these circumstances, staff were put in an awkward position; leaving prisoners in the ARU was often cited as the easiest, safest and only option available to staff, when responding to prisoners who they did not consider to be at-risk to themselves. The general reluctance to 'sign off' prisoners from the ARU was also reinforced by a commonly cited risk averse culture within both prisons:

[the Corrections officers] are worried about the risk that they take if they don't put them at-risk and they hang themselves... It's high-risk, you make a mistake like this and it goes to the paper and as much as you don't want to, you do end up taking the can for it... So the officers, because of the culture of the department, the risk averse culture, they don't want to be the ones to say that he's not at-risk and then he hangs himself (Nurse, HBRP).

They know what to say, when to say it and because of the systems we have in place we are sort of bound with what the prisoners are presenting, even though we might think he's full of shit, and we know he's manipulated the system, but we just can't get round that... But you've got to play on the safe side too, because we've had three deaths in custody in the last year, so you'd be a fool not to listen... So it's a catch-22 for us at the moment (CO, RP).

A CCDHB worker based in HBRP spoke of how the Department of Corrections' desire to mitigate risk was more focused on reducing a prisoner's risk of reoffending, rather than reducing a prisoner's risk to self. Viewing risk in a highly individualised way, as well as the existence of competing risk priorities, led to constraints for forensic staff and the doubling up of work when managing forensically challenged 'at-risk' prisoners:

Psychology is important - that's psychology to treat the mental illness. Psychology to treat the offending, all these guys need that, that's offered by Corrections, but Corrections don't treat mental illness things, they treat the offending... It's completely split, to the extent that there's a barrier of confidentiality in [the psychologist's] reports and our reports. There's an overlap obviously but we're not allowed to use that unless we get consent. And more often than not the guys will usually give consent, but they're very anxious about their offending and about getting into more trouble. So you've gotta respect that and I'm not there to get them into more trouble so I don't really want to know stuff that's going to get them into trouble, that will make them feel like they're going to get into trouble. I deal with the problems of well-being and anxiety and depression and stuff like that, I don't want to cause them more anxiety by delving. But at the same time I don't want to double up on work, or get two lots of psychologists trying to treat them for bad recollection of childhood, what's the point in that? (CCDHB worker, HBRP).

These examples demonstrate how disciplinary perspectives can lead to a narrowed view of risk that is not always helpful to the progress of treatment, care and rehabilitation. Given the importance of psychologist's reports in parole decisions, it is 
essential that prisoners have independent sources to work with when disclosing mental health and other related concerns (Connolly \& Ward, 2007). It is worth noting that some Corrections Officers work in ways that mirror health professionals, and vice versa. However, there does appear to be some tendency for professionals to view ARU prisoners on the basis of their previous working knowledge or ideologies. For health practitioners, traditional working cultures prioritise the prevention, treatment and recovery of illness, including of mental illness, while correctional staff cultures are centred on the management of risk security and the safe containment of prisoners.

\section{Risk and the working context}

Risk-averse cultures can be compounded by other occupational constraints that, more often than not, lie outside of the control of ARU staff. For example, all staff interviewed, across both prisons, raised the lack of specialist mental health and 'at-risk' training among custodial staff as a significant issue. When staff were trained, expertise was not retained, due to frequent staff rotations in and out of the ARU. Untrained or inexperienced staff working in the ARU were described as being incredibly risk averse and cautious when working alongside 'at-risk' prisoners:

We're having a lot of new staff come through, there's no training, which the PCO wants to start doing again, so that people have a better understanding. And there's an element of panic when somebody's unwell here - 'quick, call the nurse!' Which is fine, but they're in a place where they're supposed to be cared for. They can't hurt themselves in here generally, so the urgency doesn't have to be so great (Nurse, HBRP).

Further, HBRP ARU staff spoke of how they felt an increased sense of hypervigilance and that 'at-risk' prisoners faced increased risks as a result of the Unit's overcrowded and overused nature:

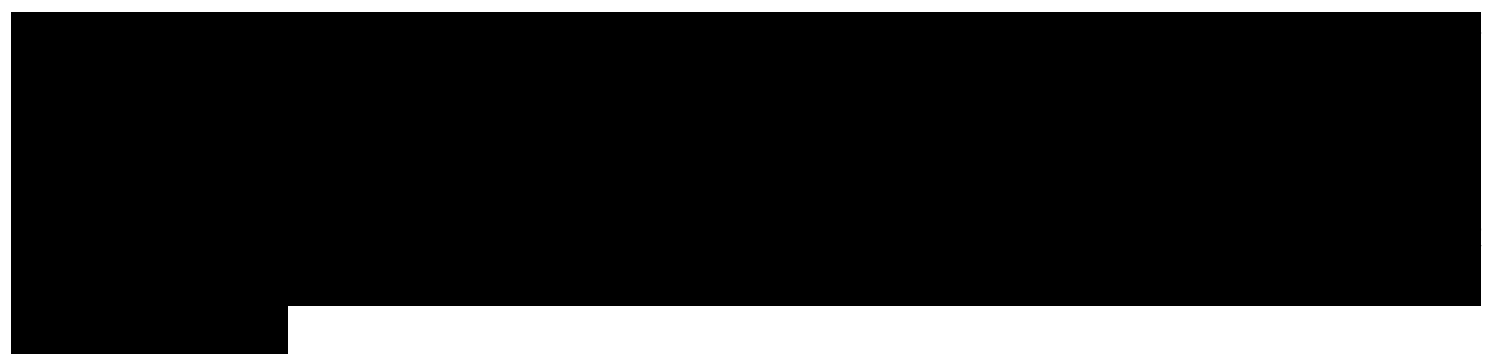


Similarly, in RP, Corrections officers were more willing to allow greater weight to be given to the advice and expertise of forensic and nursing staff, in the interests of mitigating risk to prisoners and subsequent institutional fallout or blame:

[Custody] have the final say - they can override us, but they choose not to because - I don't know if it's because we're health or if it's what we've been trained in, I'm not really sure, but they just listen to what we have to say and respect our knowledge really. Like the PCO has said, I can over-rule that - but then that's your choice if you do that - but I'm going to document that I would like that prisoner to stay here...[if] you override that then that's on you (Nurse, RP).

In sum, the ways in which staff are prepared for ARU work - and their previous training in the area of mental health in particular - can have a significant impact on perceptions of risk as well as responses to 'at-risk' prisoners. It is also evident that a lack of confidence to deal with ARU prisoners is intensified by siloed working practices, which will be expanded on in Chapter Six.

\section{Summary}

While protecting the prison from risks at an institutional or organisational level is certainly important to staff, they are also motivated to protect themselves against personal blame. At the same time, many staff care about prisoners and want to protect their lives and well-being as well. One CCDHB worker noted:

I think that every person suddenly starts becoming anxious about the risk on themselves. I think that there is also empathy with the person, that you don't like to see somebody so distressed, but it would be silly to say that we don't all feel a degree of anxiety, that this would cause enormous problems for ourselves, if this person kills themselves. I mean the person may be completely obnoxious but you still don't want them to kill themselves. You want them to sort themselves out, that's what you want... And so I think people do become much more anxious if they feel that there's a risk to their own livelihood or situation, or threatened by the person... But I think the level of distress is probably the most immediate drive (CCDHB worker, HBRP).

Despite a clear focus on risk existing within the ARU, an obvious human side to suicide and self-harm also exists; prisoners are not always viewed as simplistically as liabilities or 'risk factors' (O’Malley, 2010: 1) by staff:

And even if you had been rolling around the floor the day before, because he had been assaultive to an officer, when you open the door and see someone hanging you see a person - not a prisoner, not the idiot that was shouting and swearing at 
you two weeks ago - you see a person. And it does affect staff, because when it comes down to it they're a person too (CO, RP).

Given the commitment of staff, and their wish to see prisoners genuinely improve, it is important to find ways to enhance ARU activities, by ensuring risk management is not driven by hollow, compliance focused approaches to prisoner care. While the official policy of ARUs is largely framed around risk management, as highlighted by the findings in this chapter, ground-level working cultures can lead to different interpretations and manifestations of risk-based policies and assessments which are not always punitive or exclusionary in nature. Generally, blanket applications of policy should be avoided, particularly given that the current purpose of both ARUs is clearly much wider than just those immediately or significantly at-risk of self-harm or suicide. This chapter has also highlighted that at a policy level, risk discourses can run counter to a health-based approach to prisoner care. In turn, one significant route towards more humane, less restrictive ARU practice is through the strengthening of MDT workings. The next chapter will further unpack the ways in which the input, and convergence, of distinct disciplinary lenses in practice can help to counter negative or exclusionary risk practice. 


\section{Chapter Six: Multi-Disciplinary Teams and Ground-up Pathways to}

Care

Multi-disciplinary teams (MDTs), typically comprising correctional, medical and forensic personnel among others, have become a central part of correctional suicide prevention policies across several jurisdictions (see Corrective Services NSW, n.d.; UK Ministry of Justice, 2013; Justice Health, 2014). The use of MDTs brings correctional practice in line with community approaches to suicide prevention (Ministry of Health, 2006) and their use can bring positive outcomes. They can: challenge poor communications among staff (Hayes, 2011); generate more humane, non-punitive responses to those who are mentally unwell or 'at-risk' (Adams \& Ferrandino, 2008); and, provide a broader base of support for prisoners, leading to more self-disclosure, and greater continuity of care (Mills, 2002; Liebling et al., 2005). Research indicates that the most successful MDTs are well resourced, have open channels of communication and operate within progressive, non-punitive cultural environments (Liebling et al., 2005).

At the same time, taking an MDT approach to 'at-risk' prisoners illustrates 'the treatment-custody conflict' (Adams \& Ferrandindo, 2008: 916). Studies highlight how punitive or control oriented prison cultures can subsume healthcare approaches, which can in turn foster negative staff interactions with prisoners (Sim, 2002; Crawley, 2006). Further, prison healthcare staff can feel professionally isolated as institutional emphases on control or risk management overrides their care for prisoners (Birmingham, 2003). These conflicting approaches can cause conflict between staff, limiting the care-oriented value of MDT practice (Ramluggun, 2013).

This chapter reflects on this existing literature by exploring the practical working dynamics of healthcare staff, including both external CCDHB workers and Departmentemployed nurses, and custodial staff in New Zealand's ARUs. In particular, it considers how institutional and disciplinary cultures, including the attitudes that staff harbour towards prisoners and each other, influence MDT operations. It also discusses how organisational and practical constraints, such as resourcing and training, may inhibit 
good MDT working practices. The chapter concludes by highlighting positive practices within the RP ARU, where a cohesive MDT dynamic has led to care-oriented ARU practice. Such developments can provide valuable guidance to the policy and practice of other ARUs throughout New Zealand.

\section{The working dynamics of custody and care}

The disciplinary cultures of 'custody' and 'care' guide ARU practice. Staff often spoke of the existence of differing organisational priorities and cultures among healthcare and custodial staff:

There are different cultures - it's very clear. And that just arises out of the training - to get to be a Corrections Officer you have this training course, and to get to be a nurse you have this training course - and everything about it is different (CCDHB worker, RP).

On occasions we butt heads, we do have different priorities (CO, HBRP).

Custodial staff from both prisons recognised the existence of a disciplinary divide, but also described their role as being less clear cut. As discussed by Liebling and Price (2001), prison officers often face competing occupational demands in the course of their work, which can be difficult to balance. Given the level of need among prisoners in the ARU, many custodial staff felt as though their 'custody' and 'care' functions overlapped:

Our primary job, it's custodial. But then again, it's more than that - it's that caringness. The officers do care about the prisoners in here, they do worry about the prisoners in here. They're always looking out for signs of change and stuff like that (CO, HBRP).

It's very hard to divide it... We have an emphasis on containment but we have an equal sort of emphasis on caring and looking after them, we can be tough when we need to, and we can be soft where it's appropriate (CO, RP).

Conversely, some custodial staff felt less comfortable taking on 'care' functions, noting that a blurred mandate could create inconsistencies and issues down the track:

Are officers best placed to counsel? Coz one minute you're having to say 'no' and... you're grappling with them and fighting with them, and then the next minute you're playing games with them, you know. I think there needs to be a little bit of demarcation there (CO, HBRP). 
Health staff also noticed how custodial staff had to balance custody and care priorities, and would do so to differing degrees, depending on the context and the individual staff member at hand:

I think some officers are too caring and I think sometimes the prisoners rule the unit. But then sometimes that caring officer is the one that manages to break through the walls if prisoners are being really, really defiant, so it's a very fine line (CCDHB worker, HB).

While caring correctional officers were not ostracised by other staff for appearing weak or soft in either ARU, as indicated in previous research (Sim, 2002), some health staff highlighted how a 'soft' approach could also bring problems for correctional staff and the Unit.

The balance of custody and care priorities was also apparent in the approaches of health staff. All healthcare staff recognised that their primary duty was to provide care for prisoners. However, some health-based staff viewed prisoners as 'less-eligible' or lessdeserving of care (Sim, 2002: 300). This view towards prisoners was particularly evident in HBRP:

They're really big on this community, community, community, and prisoners have rights and all this sort of shit. But some of these people don't wanna be helped.. And some of these people don't wanna change... Some people don't quite get it...

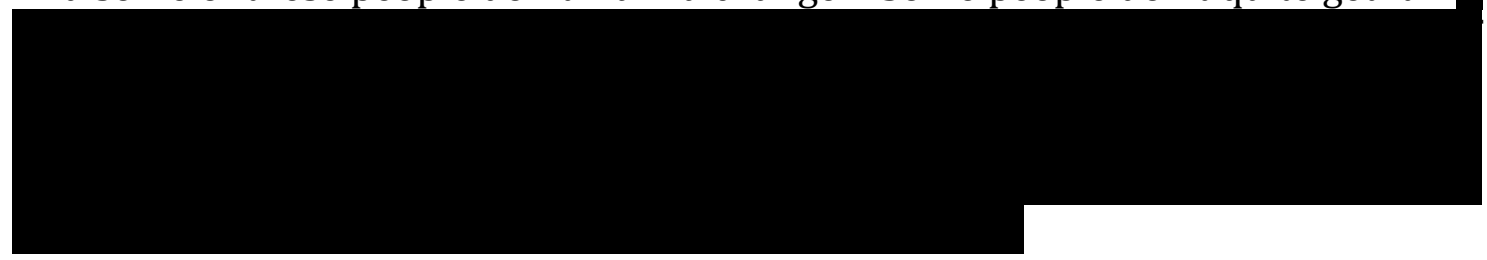

I don't mean to sound harsh, but prison is prison... We can't go flowery... The only reason they are here [is because] they've put themselves here. See you say therapeutic and I just think luxury ya know, and why would you do that? (Nurse, HBRP).

These examples highlight how traditional disciplinary approaches can sometimes become blurred, or be influenced by a wider culture that conceptualises prisoners as 'malingerers' or manipulators (Sim, 2002: 302). They also illustrate that staff from different organisational backgrounds do not always fit their own 'disciplinary mould', whether it be care or custody oriented. Some staff felt that nursing staff might arrive with a culture of 'care' but could become institutionalised into a wider correctional culture of control: 
You go out to Corrections and whilst [the nurses there] are health focused, you have to do 'getting got' [courses]. And you have to do things that are specifically Corrections based, and you have the officers doing the 'Oh this one's just trying it on', or...'This one's been hiding his medication and we can't do that', 'That one swore at you so you need to charge him'. And after a while that becomes the way that you function because that's the norm. And whilst I don't think that the nurses lose sight of the healthcare needs, they are so used to having their chains yanked by people that don't need it, or people who want drugs or whatever, that when it comes down to the people that do genuinely need it, sometimes they miss it (CCDHB worker, RP).

In sum, these excerpts highlight how custodial staff are not always inherently punitive and how healthcare staff can also propel the security-driven correctional mandate and culture. These findings reinforce the importance of culture, as a guiding principle for MDT workings, while also acknowledging the varying approaches to prisoner care that exist across correctional sites.

Despite these examples, staff generally described a more humane or caring approach towards prisoners within the ARU, in comparison to the mainstream prison:

I think it maybe is the ratio [of prisoners to staff]... Maybe the types of prisoner, because they're deemed at-risk they have to be treated differently - you can't be like 'Come on! Fucking move it!' ya know, it's more like 'Come on, in your cell, let's go'. A bit more softly-softly (Nurse, HBRP).

Furthermore, staff from all disciplines spoke of having a vested interest in the smooth running of the ARU. As a result, custody and care priorities were often seen as symbiotic rather than inherently in conflict and traditionally competing priorities could often converge in the course of ARU practice:

A health agenda and a custodial agenda are not the same and the ARU is probably the place where that is the least obvious. Because in the ARU the custodial agenda has come to realise that the person's at-risk. And they want that risk dealt with and we can help. So we have the most co-operative relationship in the ARU with custody that you can have in the prison. In other areas, I would say it's not nearly as good (CCDHB worker, HBRP).

Unpacking this further, many staff members conceptualised custody, or safe containment, as being a necessary precursor to the administration of care. The provision of healthcare was always viewed as a secondary consideration to the maintenance of security and good order in the prison. This was exemplified further by the fact that nursing staff were always accompanied by custodial staff when conducting 
daily interviews with prisoners. While justifiable in some circumstances, such an approach was seen to inhibit disclosure among prisoners and was considered a barrier to assessments of a prisoner's mental wellbeing and suicide risk:

You've got very limited time...and you have to assess them in front of officers, at least two, and sometimes I think 'how can you engage?' It's a very difficult environment to engage with them (Nurse, RP).

Because [prisoners] feel that that can be intrusive, that custodial are listening to their problems and they don't feel like that is right. And some worry that that is going to go somewhere else, or that it'll be file noted and that it'll go against their parole. So some of them are quite scared (Nurse, RP).

In relation to this, custodial staff also highlighted how their uniform and position was often a barrier to 'connectedness' and that prisoners viewed them as 'pigs, screws', as 'the enemy, to a certain degree' (CO, RP). On the other hand, forensic staff, who did not have to be accompanied by custodial staff, reported much higher levels of prisoner disclosure and openness. CCDHB workers from RP highlighted how their relative independence ${ }^{33}$ also enabled greater levels of communication and trust. Prisoners could speak freely, and because 'you're not in a uniform and you're not part of Corrections, it's actually quite helpful for the person' (CCDHB worker, RP).

The view that healthcare was secondary to custodial duties was also evident in responses to volatile or aggressive prisoners. In these instances, prisoners were regarded as a barrier to care in themselves, particularly in instances where staff safety could be compromised:

Forensic and health have different [priorities], but for me, if... if I don't maintain good order, [the forensic nurse] can't come in and see a prisoner because it's too dangerous... The order part is needed before he can get his medical care - which we're not denying him, we just might be postponing until he's not as elevated (CO, $\mathrm{RP})$.

Volatile or disruptive prisoners were more likely to be treated as a security concern by staff, rather than an as a distressed individual with immediate health-based needs. In these instances, prioritising the containment of mental health problems may undermine the possibility of administering care-oriented responses to 'at-risk' prisoners. In recognising this conflict, some thought that the ARU would benefit from greater input

\footnotetext{
33 Psychiatrists and forensic nurses are employed by the DHB and externally contracted in to the prisons.
} 
and oversight from health staff, given the specialised needs of those held in the ARU. This view was particularly evident in HBRP:

I don't think that ARUs should be run by custodial staff... The security yes, but the focus no (Nurse, HBRP).

In sum, the differing organisational priorities of healthcare and custodial staff provide useful contributions to the daily running of the ARU, despite forensic and other health care often being viewed as a secondary consideration. This section also highlights how disciplinary cultures are not always clear cut or essentialist in nature, which may cause issues for 'at-risk' prisoners and a care-oriented approach to ARU, particularly when punitive views towards prisoners are harboured by staff.

\section{Occupational constraints and organisational barriers to care}

Pressures on staff can directly inhibit more caring approaches to prisoners within the ARU. Interviews with all staff highlighted that when staff are time-pressured or resource-stretched their focus turns from prisoner well-being to the successful completion of the tasks which constitute their primary occupational duty. Two staff members from HBRP noted how, if it were not for time pressures, staff were generally proactive in taking a humane and caring approach to prisoners within the ARU:

[The staff here are] actually quite good hearted people. And it's usually the pressure of work, when the place is full with 20 people or whatever it is, that would limit them from paying attention to certain individuals. When you just leave them to interact with inmates who are distressed they will often do things off their own bat...Like 'we really thought he needed a shower, we really tried to get him some extra food, or we tried to talk to him'. They'll often do things that are quite...kind (CCDHB worker, HBRP).

When [custody staff] are time pressured they've gotta get people out, so they'll treat people...as prisoners, if they've got a less lot of them they'll treat them as patients (Nurse, HBRP).

Both nursing and custodial staff spoke of being 'rushed off their feet' when attempting to complete administrative duties and other routine tasks. Custodial staff based in the ARU were responsible for showering prisoners, providing meals, carrying out observations, attending meetings and writing up paperwork, such as incident reports, prisoner complaints and daily 'at-risk' prisoner updates on IOMS. They described such duties as being time-intensive. Staff were frequently told to document, document, 
document. So that if the shit ever hit the fan at least you have given good reasons why you didn't put them at-risk' (Nurse, HBRP). Staff talked about record keeping as being a large, but necessary, part of their daily work, in order to facilitate information sharing but to also mitigate the fallout of future risk.

While many custodial staff found working in the ARU to be stimulating and satisfying, many saw it as a challenging environment:

There are normal days. Most days are normal days, and I will describe them as chaotic. It's a very busy unit... Especially when we are as full as we are at the moment... It's busy. It's always busy (CO, HBRP).

We do everything that every other Corrections officer in the jail does, plus we work with forensically challenged prisoners... Although we get quieter days, I work harder in this unit and I'm more mentally drained in this unit, working with 7 prisoners out there than I ever did when I had 44 prisoners double bunked in a remand unit (CO, RP).

As noted in Chapter Four, the busyness of the unit could also adversely impact on prisoners and their daily experiences. For example, one custodial officer noted:

We cycle through the prisoners, give them their hour out. If there's more prisoners they obviously get a little less time out, coming up to the lunch hour, if we've got less then there's more time out (CO, RP).

Nursing staff are scheduled to visit the ARUs twice a day, in order to conduct brief interviews with prisoners and to distribute medication. Similarly, the interactions between nurses and custody staff in HBRP could inadvertently become strained as a result of the busy pace of the ARU:

I guess the biggest challenge is trying to get the officers time to do a handover, coz they're usually answering phone calls, letting people through the doors or they're busy giving prisoners showers... They do sometimes ask you to hurry along. We've had officers that will start shutting the door on you as you're talking, because they are time pressured and they think sometimes you talk too much (Nurse, HBRP).

Such issues were compounded by other staffing problems (Mills, 2002; Liebling et al., 2005). Staff across both sites discussed staff retention issues, within both the ARU as well as the prison more broadly, as a key pressing issue or challenge. Resourcing pressures were particularly problematic in HBRP, given the high numbers of ARU prisoners. HBRP Corrections officers commented on how short-staffing compounded 
the stresses and administrative burden of ARU work, while also limiting opportunities for therapeutic interventions or other care-oriented services:

We've got a gap on our roster, an unfilled line, so we're running a staff member short... And they decided it's our turn, so they're not going to fill that slot... Which just throws more pressure and more stress on the rest of us (CO, HBRP).

We don't have time, with the number of staff here...to do extras with them. When you got 14 here you don't have time to... You're rushing and you're not focused, you're not keeping yourself safe. So if they were to have other activities then you couldn't guarantee the unit's safety... You'd have to have more staff to implement any [kind of other interventions] (CO, HBRP).

Resourcing stresses could be particularly acute in the ARU, when inexperienced staff were frequently forced to cover those with greater expertise in dealing with mentally unwell, and 'at-risk', prisoners:

I went this morning to the front gate, and the front guy came over here and worked, which makes it twice as hard on the officer on the floor... Because he doesn't work here, he doesn't know the routine. And it's ridiculous (CO, HBRP).

Despite some correctional staff being 'well suited' to working in the ARU, for example by having a genuine interest or specialist knowledge in the field of mental health, such expertise was often lost in the ARU as a result of the Department's staff rotation policy. The policy shifts staff across different units, in order for workers to gain a range of skills and experiences and to avoid staff complacency. In HBRP, rotations were described as occurring frequently, around three to four times a year, whereas in RP rotations occurred every few years. While not totally unwarranted, ARU staff saw this approach worked negatively in the ARU and also created uncertainty for workers:

I think most of the problems and the challenges are sort of ones as a Department that we create for ourselves. We have the blanket rotation policy, so you can get any staff coming through here - so you quite often get staff who don't want to be here or who aren't suited to being in a unit like this... And also really good staff will get transferred out as well (CO, RP).

It's very frustrating, it's demoralising... in 13 years, I had 14 moves - I was forever on the move, so that I'd just start to feel part of a team, start to feel as though I was contributing and knew the ropes, and then I'd be off somewhere else. The longest stay I had anywhere, prior to my move here, was just under 2 years, so some of those periods spent in units were quite short... It either tells me that I'm really good and everybody wants me or I'm useless and nobody does - and I haven't worked it out yet! (CO, HBRP). 
A CCDHB worker at RP, who had been involved in the running of the ARU since its introduction, noted how the ARU had previously benefited from consistent and specialised staff. With the introduction of the rotation policy, this worker had seen new challenges in the management of mentally unwell prisoners:

Often it's a personality or a couple of personalities that come in that just change the whole way that it works. That's been quite hard... I don't want to be rude, but it kind of de-skilled the ARU all in one go, and then you kind of have to start again, building up... Having staff done on a general rotation means that people aren't necessarily selected for their suitability for the ARU...And that itself creates a level of frustration when trying to engage with patients who are having difficulty with specific officers because they're difficult, not because the patients are causing the problems (CCDHB worker, RP).

Issues relating to staff rotations and lack of specialised training are intrinsically linked. For example, where staff members did receive specialist training, their expertise would be promptly rotated out of the ARU and replaced by inexperienced and untrained staff members:

The last two PCOs who were in here went off to Wellington for some sort of training in dealing with mentally ill patients... What to look for and all that sort of stuff, the day-to-day care. And neither of them is here, they went off and did that training and then they both got moved. Now, where's the sense in that? This is a highly specialised unit and you would think that they would train people, give them the training they need - which I haven't had - and hang on to them as long as possible - Nah. It flies against common sense (CO, HBRP).

The lack of training, and the lack of dedicated, full-time specialist staff more generally, was repeatedly raised by all participants as one of the most pressing challenges. While all custodial staff members received generic 'at-risk' training at 'college', the lack of specialised mental health training limited the opportunities for care-based treatment towards 'at-risk' prisoners:

It would be really useful to have some sort of directed training... Because we can make someone go off his nut without being aware that we shouldn't be doing this or saying that because we simply don't know. We do what we can do (CO, HBRP).

We don't get anywhere near enough training... as less and less beds are available [at Purehurehu] we have the backlog with more and more people with mental health issues...the staff just aren't equipped to be able to deal with the prisoners that we have up here (CO, RP).

Forensic and nursing staff echoed this sentiment, noting that custodial staff were often 
poorly equipped and under-resourced to deal with high-needs prisoners. Forensic staff in HBRP noted that, as a result, custodial staff were quick to medicalise bad behaviour:

There needs to be a huge amount of training with custodial staff that behaviour is not a mental illness... They tend to think that someone who kicks up needs to be put in an ARU, but it's behaviour, they need to manage behaviour... But they like to medicalise it and want us to medicate it (Nurse, HBRP).

Such an approach could, at times, lead to tensions and misunderstandings between nursing and custodial staff:

And [custody will] ask you 'ohh is such and such on medication?' 'No, why?' 'Oh well I think he should be, rah rah rah...' 'Really?'... Things like that cause friction with nurses and custodial. Because it's not all mental health (Nurse, HBRP).

Furthermore, there was a sense from some participants that the stressful nature of the ARU inclined custodial staff to manage volatile or rowdy 'at-risk' prisoners with medication, or via other less therapeutic means, in a bid to relieve pressures. More generally, the time-pressured nature of the daily ARU experience was seen to compromise the ability for MDT relationships to be meaningfully developed:

There can be [conflicts of interest between health and Corrections staff]. Coz they [Corrections] wanna move people out of the unit coz they don't have enough staff or they're time pressured... and the fact that we are here, in relation to them, for such a short time - they're with the patient all the time and we're only here whizzing in and whizzing out, making our decisions - so, they'll say 'that guy said to you that he was self-harming but he wasn't - I've seen him all day and he's fine' (Nurse, HBRP).

This excerpt highlights how occupational pressures, such as a full ARU, can become exacerbated when specified time is not put aside for MDTs to discuss a prisoners needs, particularly when staff present conflicting viewpoints in relation to a prisoner's 'at-risk' status. Moreover, staff relations can become tense and MDT working can become increasingly siloed and marginalised when the ARU is time and resource pressured.

In summary, while health teams can be a valuable source of support for 'at-risk' prisoners (Liebling et al., 2005), their contribution can be undermined by cultures of compliance, notions of 'lesser eligibility', inadequate training and siloed working. This section demonstrates that when ARU work is dominated by paperwork or other routine-oriented tasks, and when the ARU is full, prisoner care can be diminished. Further, staff become too time-stretched to properly integrate and build connections 
across disciplinary teams. Resultant breakdowns in communication and misunderstandings about mental health needs, can lead to 'rifts' between teams (Nurse, HBRP). Such rifts can become exacerbated when staff feel burnt out or unsupported in their daily work. The next section highlights how RP's MDT, despite experiencing similar issues, benefit from greater resourcing and fewer numbers within the ARU. The RP experience shows that management and culture have a significant impact on staff ability to foster more care-oriented practices.

\section{RP's ARU: Towards a successful MDT model}

Staff at RP grappled with many previously stated 'occupational' issues, such as shortstaffing, inadequate training for custodial staff and generally stretched resources, which impacted on positive working practices. However, despite these shortcomings, the ARU was governed by a highly collaborative MDT. More generally, the ARU operated a 'climate of support' among staff, which gave rise to several creative ground-up initiatives that invoked positive outcomes for both prisoners and staff. Long serving staff spoke of how enhanced practices emerged from deliberate changes to ARU practice, suggesting that similar changes could develop in ARUs elsewhere. This chapter illuminates the positive working practices of RP's ARU, with a particular focus on how positive MDT dynamics underpinned other emerging good practice.

\section{Daily meetings and informal connections}

Unlike HBRP's ARU, that held weekly MDT meetings, a range of multi-disciplinary staff in RP's ARU meet every morning at a set time to discuss 'at-risk' prisoners who are 'up for review'. These daily meetings are not set out in policy, rather they developed organically, 'over the last three to four years' (CCDHB worker, RP), as a way to keep all staff aligned and informed about prisoners within the ARU:

You have to support each other, so I think that since we've been having these daily meetings the work that we do in the ARU is changing - it's not only in and out, we're able to have more open discussions. Years ago it was just in - do your work - out, never had meetings. The PCO just used to read what you had written and you'd say 'happy for him to be signed off' and that would be the whole of our conversation. Now...there's more to it (Nurse, RP). 
Daily 'check-ins' were viewed as beneficial for staff as they provided a direct and immediate channel for concerns to be raised and updates to be shared with everyone in the MDT. Beyond the meetings, informal relations were strong and staff worked diligently to maintain positive relations with other teams. CCDHB workers discussed a growing sense of informal integration and support between custodial and forensic staff, which also proved to be useful:

I think that what we've focused on and what's worked really well is the day-to-day kind of liaison that occurs on a case-by-case basis... I think that's what makes the most difference actually (CCDHB worker, RP).

It's really important to go and have cups of tea over there... to try and be part of the team, because you actually are part of a team, and if you're not there they can't ask questions and often, particularly with the newer officers, it takes a while to realise there are no silly questions (CCDHB worker, RP).

The physical proximity of teams in RP's ARU was of huge benefit to positive MDT workings. Unlike HBRP, where the Health Centre was separated from the ARU by a few hundred metres, the RP ARU was directly adjacent to their respective Health Centre and medical staff. Further, unlike HBRP, forensic staff who worked on site were based in the ARU rather than in another area of the prison. Corrections officers in RP thus had open access to forensic staff and did not have to get through 'red tape' or arduous 'chains of command' (CO, HBRP) in order to have forensic issues addressed. This close physical proximity to custodial staff was helpful in building rapport, more generally:

More often than not we have that desk in doorway and staff will regularly come and plonk themselves in the chair and discuss stuff about patients or discuss things that might be an issue with them, the unit, in terms of somebody that is causing frustration or whatever, or talk about their own staff stuff which is good as gold - but they very much treat us like part of the team (CCDHB worker, RP).

The proximity to nursing staff also facilitated positive working relations among healthcare and custodial personnel:

If we've got a query and they're not there then we'll just pop down, we've got a very good working relationship with the nurses here because obviously we're right next door... They pop in when it suits them, which suits us as well, and the door's always open... If I need to speak to the manager then I can just walk through and knock on the door and speak to [them], no worries, and that's a really good thing (CO, RP). 
These excerpts highlight the extent to which staff at RP value strong, well-integrated teams, which as per other successful MDT workings, was often fostered or underpinned by proactive leadership and management across all teams (Liebling et al., 2005). While RP's ARU staff were certainly busy, it is worth noting the comparatively less full ARU provided more opportunities to focus on collaboration and communication among staff members.

\section{Shared expertise and culture of education}

The RP ARU exhibited a culture of education and information sharing among all staff. Custodial staff were encouraged to ask questions and take a proactive approach to learning about mental health and other behavioural concerns:

The forensic nurses are very good... They're very open, or as open as they can be. Like this morning [the forensic nurse] was talking about manic and I wasn't really sure what manic meant, pertaining to the prisoner, and she just broke it down for me and what the deal was and what he had been going through and so it opened my eyes a wee bit too, in terms of what the prisoner was experiencing and how maybe I could better manage him or how to keep myself safe, so nah we've got a really good relationship with them, I think it's great (CO, RP).

Similarly, CCDHB forensic staff had a vested interest in building up mental health expertise across the unit:

The officers who work in the ARU are quite skilled and interested in doing that work, and as we support them on a case-by-case basis with difficult people, they develop quite a high level of expertise actually, as do the medical staff, and we're in the business of supporting them as well... We are trying to set up a series of visits so that Corrections staff can come and see how it works here and so our staff can go and see how it works in Corrections, in the ARU in particular. So some of those barriers have been broken down over the past few years (CCDHB worker, $\mathrm{RP})$.

Rotations in RP's ARU seemed to occur less frequently than in HBRP and, as a result, the educational investment in custodial staff was retained to a greater extent. Despite custodial staff holding clear views about formal training being insufficient, these more informal educational networks were seen as useful and worked to bolster the confidence and autonomy of custodial staff based in the ARU. 
Official policy states that ARUs are led by the PCO and their custodial teams, alongside advice and assistance of the onsite nursing team and external forensic staff (Department of Corrections, 2012c: M 05.03). However, in practice, forensic and nursing staff in RP's ARU had equal, if not greater, input to the management of 'at-risk' prisoners than custodial staff, who described themselves as 'just the gatekeepers' (CO, RP). While health staff acknowledged that the PCO still had the final say, they noted that in practice it was rare for custody to undermine the input of health:

[Custody] have the final say - they can override us, but they choose not to because - I don't know if it's because we're health or if it's what we've been trained in, I'm not really sure, but they just listen to what we have to say and respect our knowledge really (Nurse, RP).

Similarly, custody respected and valued the expertise of healthcare staff in relation to determining the management of prisoners with mental health concerns:

The bottom line is health and forensics. If health and forensics want him down here he stays here... If it's a placement issue or something like that then we can work alongside the prisoner, it's in everyone's best interests to stop them bouncing back, but pretty much when health and forensics say it's time to go, or that they no longer need to be here, then they get signed off (CO, RP).

With the ARU being effectively led by health-staff, the culture of care and support which had been adopted by the healthcare unit (which heavily contrasted with the nursing culture of HBRP) was able to support more individualised, tailored responses to 'at-risk' prisoners:

I think it's the staff, I think it's the changes over the past few years that have come in, I think the nursing culture has changed within the prison as well... We're not adopting the custodial hard line like we used to, and that's why I refer a lot to patients - years ago it was prisoners - you are a prisoner, you've come in here well they're not. They are prisoners, yes, but we're health staff and we're here to treat, not to punish, so that whole culture of thinking is changing (Nurse, RP).

The sense of joint accountability and high levels of trust within MDTs seemed to be bolstered by strong systems of verbal information sharing, for example during the daily morning meetings. Multi-disciplinary staff could bring different sets of facts to the table, helping to paint a holistic picture of a prisoner's mental state. Such an approach provided for a robust system of checks and balances. Information sharing meant 'at- 
risk' prisoners could be 'managed out' of the ARU in a timely and suitable fashion and also meant that 'at-risk' behaviours were not missed:

We see the guys daily, whether they're on medication or not, we have conversations with them, finding out how they are, how they're feeling, where they're at... Once all of that is completed we will talk to custodial and see how they've been finding... We can sometimes have a different view - either they're being more open with custodial or they're being more open with us. So we have a tight working relationship. We have to for the safety of the patient (Nurse, RP).

The thing is with prisoners... They put on the big brave face coz 'I'm the man', they sit there all staunch and then they see the first female nurse and they start crying and they'll want to end their life. And I guess that's the robust system that we've got in place, we hopefully catch it before they get down to the units (CO, RP).

More generally, staff seemed genuinely interested in ascertaining prisoners' health needs as opposed to minimising their risk in a managerial sense. As highlighted in the previous chapter, such an approach enabled staff to prioritise the interests of the individual prisoners over rigid compliance with official policy. Multiple staff spoke of taking extra time out to properly understand and resolve individual prisoners' issues:

When [the prisoner] first came through the officer said he was fine and then with my experience [in mental health], I was talking to him and I felt like something wasn't quite right... So I actually purposefully spent more time talking to him and then eventually he burst into tears and I said 'you're not alright?' and he said 'no I'm not' (Nurse, RP).

We've just had a patient who's moved to Purehurehu on Monday I think it was, and we would have a fortnightly meeting about him with the unit manager, the PCO, the SCO, myself, the Health Centre Manager, the nurse doing the ARU, his psychiatrist and the forensic nurse - a big meeting, and we would be having that once a fortnight just about where he is, what we can do for him, the behaviour is getting better or worse - those meetings can be half an hour to an hour dependent, we've done that a few times (Nurse, RP).

Furthermore, as noted in the previous chapter, MDT staff collaborated with prisoners and asked them directly about their needs, making management and recovery an inclusive and non-coercive process (Sim, 2002). Taking a more holistic, needs-based approach meant that prisoners were less likely to immediately 'bounce back' to the ARU and, when necessary, tailored supports were put in place to aid successful integration back into the mainstream prison environment. 
In HBRP, and previously in RP, staff reported that prisoners frequently 'bounced back' to the ARU once they were returned to the mainstream. One way RP staff worked to minimise this from happening was via the integration and involvement of staff from other prison units, when managing and transitioning 'at-risk' prisoners. One CO from RP summed up this ground-up practice well:

[Units] 13 and14 do a fabulous job of looking after our segregated prisoners who have mental health problems, and the staff are really interested, and they've become really involved... And they've had massive successes. And what they were doing, with some of the patients, is a staff member from the ARU would take them down to 13 and 14, spending a few hours down there with them...making sure they were doing OK...and then once they were down there if any problems came up an officer that they knew would go down and check how they were doing... So they weren't flung from something that was really quite safe and quite stable, where they knew what was going on and everything, into this environment that was really quite frightening... And then the officers down in 13 and 14 pick up on that, develop these relationships with them, get to know them really well and I mean I've been so impressed with them... They've really stepped up. If there were a step-down unit that would kind of be it (CO, RP).

Other RP ARU practices that had developed from the ground-up include the use of a negotiator in instances where vulnerable prisoners were reluctant to leave the ARU or were perceived to be feigning 'at-risk' symptoms, a common issue that HBRP staff grappled with:

We had one that was at it, we all knew he was at it, but he knew what to say...I thought let's play it differently. So the Unit that he was going to, we got the manager and one of the negotiators that we have...they would come up for a couple of hours a day to get him to go down to the unit - two PCOs, two and a half hours on two days to talk to a prisoner - is that bad management? We didn't use C $\& \mathrm{R}^{34}$ and nobody got hurt, and he went down to the unit. We all knew that...he was just hiding up here, but again if you say 'I'm going to kill myself if you move me out of it' you've got to take it seriously... So we used a different method for that. Forensics had no concern, health had no concerns with him, we had no concerns with him - for self-harm - but what was the point in signing him off and sending him down to a unit for him to just bounce back? Use something different... Get the people up to talk to him... And yeah it gets frustrating, but the result was good and he's been away and hasn't come back (PCO, RP).

\footnotetext{
34 Control and Restraint.
} 
This example, while time and resource intensive, highlights the more considered and organically developed approach to 'at-risk' care and management among staff in RP's ARU.

\section{Summary}

This chapter highlights the divergence in ARU practice across sites and the importance of 'ground-up' initiatives and collaborations to ensure a positive, care-oriented style of ARU management. More specifically, it establishes that MDT workings are heavily informed by organisational and cultural factors, as well as levels of trust and leadership within MDTs. The positive working practices of RP's ARU highlight how a highly cohesive, integrated MDT can give rise to better supports for 'at-risk' prisoners and help to successfully triage individuals to necessary services or back into the mainstream, as opposed to treating the ARU as a longer-term 'dumping ground'. With fewer prisoners being held in the ARU, and for shorter periods of time, staff can spend more time on resolving individual prisoner's needs. Furthermore, a sense of trust and support among staff in RP's ARU has allowed for unique 'ground-up' initiatives to develop on their own accord, despite not being present in any official policy - for example, the use of negotiators and the involvement of quasi 'step-down units', which will be discussed in more detail below, within the wider prison environment. The next concluding chapter reflects on these points, while also drawing together previous findings, to consider the extent to which staff can converge or collaborate to enhance the experience of 'at-risk' prisoners as well as prison staff in New Zealand. 


\section{Chapter Seven: Conclusion - Thinking about Change}

This thesis has examined the working dynamics of two ARU sites in New Zealand, from the perspective of staff, highlighting the complexity involved in administering careoriented practice towards 'at-risk' individuals within the prison environment. Staff play a central role in responding to the wide-ranging needs of 'at-risk' prisoners (Liebling, 1992); however, wider correctional constraints, priorities and mandates can often overshadow recovery or needs-based approaches to prisoner care. The challenge of administering healthcare and other care-oriented practice in a prison environment is a topic that has received much discussion and research attention (Sim, 2002; HM Inspectorate of Prisons, 2007; King, 2012; Wakem \& McGee, 2012). This thesis contributes to that knowledge by demonstrating how both healthcare and custodial staff conceive and administer care-oriented practice within a unique 'risk-focused' prison environment. This research highlights the centrality of 'on-the-ground' culture and the important role that cohesive, collaborative and well-resourced MDT workings can have on improving the ARU experience, for both prisoners and staff alike. Some of the 'ground up' learnings exposed in this thesis may benefit from recognition at a policy level and could be shared across ARU sites in New Zealand, as a way of enhancing practice and ensuring consistency at a national level.

This concluding chapter summarises and expands on some of the primary arguments raised in the main findings and posits some final thoughts on possible ARU reforms. Specifically, the chapter will point to some areas of practice and policy that could be enhanced by minor or incremental changes to New Zealand's current framework, drawing from both staff accounts and international learnings as a guide. It will also briefly raise some wider questions about the extent to which incremental ARU reform can really enhance care-oriented practice, given the constraints to care inherent in the wider correctional context and beyond. In particular, as highlighted, ARU practice raises a number of concerns surrounding the treatment and containment of mentally unwell prisoners, many of whom 'ought to be cared for in hospital' (CCDHB worker, RP) and who cannot have their treatment needs met within a correctional environment (Adams \& Ferrandino, 2008). 


\section{Summary of findings}

\section{Current ARU practice}

The findings of this thesis confirm many of the issues raised in the existing, albeit scant, literature on ARU practice in New Zealand. As identified by Roguski \& Chauvel (2009), ARUs are used frequently, for a range of purposes and sometimes for long periods of time. This thesis has shown that such misuse and overuse was particularly evident in HBRP, where the ARU was too often utilised as a containment block for difficult or disruptive prisoners. It was described as a 'dumping ground' (Nurse, HBRP) by staff. Many staff viewed the ARU as 'a time out' unit, as it gave workers a break from having to interact with stressful or volatile prisoners, many of whom had unmet mental health needs. Staff also noted that vulnerable prisoners saw the Unit as a short-term escape from the stresses of everyday mainstream prison life - a purpose not exposed in previous research. Despite this observation, it is worthwhile noting that prisoners' views cannot be wholly deduced from staff-based accounts; this was a limitation of the current study and prompts calls for further research into the lived experiences of prisoners and their perspectives of care-oriented practice more directly, particularly given the high number of ARU 'users' uncovered in this research.

Staff shared some of the wider criticisms of ARUs raised in previous reports (Roguski \& Chauvel, 2009; National Health Committee, 2010). Many acknowledged the isolating and potentially punitive nature of the Unit and its ability to impact on prisoner wellbeing. One worker noted 'they come here and they won't play games again... Put them [in the round rooms and] they'll never come back. Unless they are sick' (CO, HBRP). At the same time, all workers thought that the ARU was useful and served a valuable purpose - although there were differing views on what its purpose constituted. Particularly in RP, where staff cited a 'climate of support', the ARU was described as having 'a level of skill, interest and competency' among staff and allowed for 'a high degree of observation' via camera (CCDHB worker, RP), which worked to the benefit of prisoner safety and, importantly, could provide a platform for enhanced care. Given the better ratio of staff to prisoners, particularly when the RP ARU was less full, staff spoke of having more personal interactions with 'at-risk' prisoners, when compared to the mainstream. Further, the ARU was viewed positively by staff across both ARU sites as a 
'stepping-stone' or introductory unit for first time prisoners, who were often particularly vulnerable (Nurse, HBRP). Despite these perceived benefits, it was clear that more severe prisoner mental health needs could not be addressed within the ARU environment, despite operating as a 'de facto mental health unit' (CCDHB worker, RP), which could only hope to contain 'surface manifestations' of prisoner distress (Cliquennois \& Champetier, 2012: 397).

Moreover, there was an overarching sense among staff that there was simply 'nowhere else to go' for some prisoners, with some referring to the ARU as 'the end of the line' (CO, RP). This was particularly the case for prisoners with severe mental health needs or who exhibited other highly challenging behaviours, such as personality disordered prisoners. It is widely known that 'finding safe, humane, and non-punitive methods' for prisoners with serious mental illness is a challenge, given correctional security, rules and control-driven priorities as well as the lack of requisite resourcing (Adams \& Ferrandino, 2008: 914). Such a view supports the work of multiple commentators who have questioned whether severely mentally unwell prisoners should be in prison at all, given the obvious resourcing and cultural constraints of the institutional environment (King \& Morgan, 1980; Mills, 2002). As noted elsewhere, staff were consistently of the view that rates of mental illness and levels of acuity were steadily on the rise in both prison sites, with external forensic resources, notably available beds at inpatient units such as Purehurehu, being increasingly stretched as a result (Office of the AuditorGeneral, 2008; Wakem \& McGee, 2012). With the last comprehensive audit into mental health prevalence in New Zealand prisons being conducted nearly 20 years ago (Simpson et al., 1999), and given the prevalence of mentally unwell prisoners cited by staff, it may be timely for the Department of Corrections to revisit the extent to which mental illness exists, and the way it is managed, within the confines of the prison. While outside the ambit of the Department of Corrections' responsibility, wider consideration may also want to be given to the resourcing constraints and capabilities of forensic services and DHBs in relation to the care of severely mentally unwell prisoners, who are often inadvertently 'backlogged' into isolating and non-therapeutic ARUs until beds are made available in the community. 


\section{The practical realities of risk}

A key emergent theme in this research was the pre-occupation with risk and riskmanagement as central drivers of ARU practice and culture. Within this, it became apparent during fieldwork that assessing a prisoner's 'at-risk' status was not always highly actuarial in process and was often reliant on the 'subjective judgements' of ARU staff (Hannah Moffat, 1999: 81). At times, this inevitable subjectivity could cause interdisciplinary tensions, particularly as many participants held differing views as to what being 'at-risk' constituted or included depending on their distinct occupational or disciplinary lens. For example, it was undecided among many staff whether prisoners who were vulnerable, such as those who risked being bullied or assaulted in the mainstream but who did not present as at-risk of self-harm or suicide, were suitable for ARU placement. Fuelled by this uncertainty of purpose, cautiousness and hypervigilance when assessing a prisoner's 'at-risk' status was rife among staff, particularly in HBRP, who would often 'err on the side of caution' (CO, HBRP) when determining ARU placement, in attempts to ameliorate the potential fallout of 'organisational risk' (Whitty, 2011: 124). In some instances, risk-driven practice and priorities could work to trump individual prisoners' needs and, more generally, undermine a care-oriented approach to practice. For instance, as discussed in Chapter Five, risk-conscious staff were less likely to deviate from 'black and white adherence to rules' and blanket applications of policy in order to provide care for distressed 'at-risk' prisoners. In HBRP, the focus on containing and mitigating risk among individual staff members inadvertently led to the overuse of the ARU and exacerbated feelings of stress, burnout and professional isolation among staff members, who often cited feelings of personal responsibility when tasked with managing an individual's wellbeing and level of risk.

As highlighted, security-based adherence to rules and rigidly applied blanket applications of whole-of-prison policies was not only viewed as time consuming, resource intensive and at times arbitrary, but also ran counter to health-centric elements of the ARU. Such issues raise the question of whether the ARU should be formally recognised as a 'specialist unit' within the prison, perhaps akin to RP's HDU, given its obvious health-centric purpose on-the-ground - a suggestion raised repeatedly by staff. One way this could be achieved is via greater input into ARU staff selection, perhaps with greater input of forensic staff, and the removal of frequent staff rotations 
within the Units. Such a shift would not give rise to issues relating to staff complacency or corruption, given the often highly transient nature of the ARU prisoner population.

\section{Positive risk cultures and MDT workings}

Despite many academic commentators presenting risk-management as an inherently non-therapeutic or individualised paradigm (Hannah-Moffat, 1999; Bullock, 2011), the thesis highlighted how risk could work in favour of care-oriented responses to prisoners when combined with a 'climate of support' and collaborative, supportive MDTs, along with a higher degree of professional autonomy and discretion. This was exposed by RP's ARU. As highlighted by O’Malley (2004), risk discourses can provide a useful platform for inclusive, non-coercive and needs-based responses towards 'at-risk' prisoners. Moreover, thesis findings highlighted how a collective approach to individual prisoner care within the ARU alleviated negative risk-centric responses. In RP's ARU there was a sense that staff were jointly accountable, rather than individually responsible, for the management of 'at-risk' behaviours and the potential fallout of such risks at an institutional level. This gave rise to less cautious and security-oriented responses to prisoners and a greater sense of trust, cohesion and unification when making decisions about 'at-risk' prisoner care.

Previous research has shown that, while international ARU policies are increasingly grounded in 'joint up' or multi-disciplinary working, there is little 'critical reflection' as to how such dynamics play out or enhance practice (Mills et al., 2012: 400). While this thesis was not comparative in nature, the divergences in MDT practice across RP and HBRP's ARU sites were, at points, stark and provided interesting insight into how MDTs can work to either hinder or enhance positive, care-oriented approaches to 'at-risk' prisoners. As highlighted by Liebling et al. (2005: 15), and supported by this current research, the success of MDTs and their ability to enhance outcomes for prisoners is closely wedded to several factors: competent and cohesive relationships among managers; receptive and supportive attitudes towards prisoners and other staff; and a separation from the 'urgency of operational pressures', such as overcrowding and staff shortages. Further, as supported by this thesis and the experience of HBRP's ARU, the harbouring of punitive or less supportive attitudes towards prisoners among staff, resourcing issues (such as turnover rates and rostering gaps), difficulties in co- 
operation across disciplinary teams and other operational tensions were all pivotal in shaping the relative success of ARUs (Liebling et al., 2005). This is important as punitive staff cultures and fragmented MDT workings can have negative implications for prisoner care, for example by inhibiting prisoner access to services and treatment (Mills et al., 2012).

\section{Reforming the ARU system}

\section{The centrality of cultural and operational factors}

As highlighted in Chapter Three, 'at-risk' policies in New Zealand and a number of comparable jurisdictions are obliged to uphold normative frameworks and human rights standards, which serve to protect the interests of 'at-risk' prisoners and lay the foundations for care-oriented policies to operate in practice. Moreover, the development of international law is placing an ever-increasing legal duty on the state to proactively provide care-oriented responses to 'at-risk' prisoners, beyond a more baseline 'death-avoidance' approach to care (Livingstone, 2000; Cliquennois \& Champetier, 2012: 397). In terms of policy, in New Zealand, the ARU framework has shifted little since its relatively recent introduction. Internationally, advances in similar jurisdictions have been limited to minor shifts, such as the introduction of increased regime activities, changes to the physical environment and enhanced interactions with staff (Corrections Victoria, n.d.; Burrows et al., 2003; National Health Committee, 2010; UK Ministry of Justice, 2013). While such shifts can by no means be described as radical or ground-breaking, and are more incremental and reform-based, New Zealand may wish to consider some of these developments when enhancing policy on care-oriented ARU practice.

While 'top-down' ARU reform is useful to some degree, this thesis has cemented the centrality of supportive staff cultures in ensuring a 'culture of rights' is embedded in order to pre-empt care-oriented practice (Genders \& Player, 2014: 427). Moreover, this thesis has highlighted the way in which care-oriented 'at-risk' practice is not always guided by, or even linked to, respective policy and operational frameworks. For example, the obvious divergences in practice across HBRP and RP's ARUs support the notion that policy is often inconsistently interpreted by staff and can be shaped by ground-level cultures or other operational pressures (Smith, 2007; Carlton \& Segrave, 
2014). Moreover, the interpretation of policy and operational frameworks is heavily influenced, or in some cases 'fudged' and manipulated, by staff as a result of on-theground pressures (Genders \& Player, 2014). As touched on a number of times in this thesis, many staff had only high-level understandings of what the current 'at-risk' policy constituted, with one worker describing the status of operational guidelines as wholly detached from practice: 'just a seal on the floor... Someone who's sitting in a bullshit castle, who's never been on the floor' (CO, RP). In sum, when considering reform to the current ARU system, due emphasis needs to be given to grassroots cultures, resourcing constraints and other operational pressures. Importantly, as highlighted by Liebling et al. (2005), the successful implementation of new 'top-down' procedures can be severely inhibited by such ground-level pressures, constraints and cultures.

Bearing these considerations in mind, both HBRP and RP developed a number of good working practices, to differing degrees, which could be shared as a way of enhancing care-oriented responses to prisoners more generally. For example, the development of strong and well-functioning MDT working relationships emerged as a strong platform for care-oriented practice to be administered, to the benefit of both prisoners and staff. However, in line with broader international findings, the development of positive working relationships was enhanced via proactive leadership and clear cultural priorities within ARU teams, rather than policy-initiated reform or guidance (Cox, 1984; Smith, 2007). In sum, the development of care-oriented practice in ARUs can be better ascertained from the collaborative actions of staff working on the floor, on their own accord, rather than what may be developing from the 'top-down' at legal or policy levels.

\section{Training and rotations}

Bearing the importance of 'on-the-floor' dynamics in mind, this thesis suggests that greater support, training, resourcing and alleviation of 'on-the-ground' pressures would be useful as a way of enhancing care-oriented practice in New Zealand ARUs. The need for specialist training in mental health, or 'psychological first aid' for ARU custodial officers (CCDHB worker, HBRP), was raised by all staff interviewed. Many felt underequipped and under-resourced to manage high-needs prisoners. Mental health training for staff serving similar roles overseas has shown to be hugely beneficial to day-to-day 
practice and prisoner management; Parker (2006, in Adams \& Ferrandino, 2008: 923) highlighted how one ten hour training course for correctional officers on mental illness led to an immediate decrease in the number of incidents of use by force by officers, as well as a direct decrease in 'battery with bodily fluids' on the part of prisoners. Informal training for custodial staff, for example education delivered by visiting forensic staff on mental health, medication and side-effects has also shown to wield positive results for staff capabilities and prisoner care (Mills, 2002).

As highlighted at RP, while recognising that they were not expected or employed to function as mental health nurses, custodial staff had benefited hugely from off-site training sessions in mental health, which included a visit to Purehurehu and other external facilities. Informal training and education between forensic and custodial staff occurred frequently, and was viewed positively, by RP staff. It was also raised as a future possibility among staff in HBRP. For training to be worthwhile, the ARU would require greater stability with staffing; forensic staff highlighted how the staff rotation policy had negative impacts on the running of the ARU and did not take into account its specialist nature.

\section{Physical environment}

A major barrier to care-oriented practice, and equivalence of care more specifically, is the ARUs physical environment. One participant described the limitations of the current setting, describing the ARU as:

An awful environment, just physically, awful. What can you change without rebuilding it?... One of the things that they're doing within the mental health field is they're trying to reduce seclusion, as much as they can, so you have a right to be around others and you have a right to having company... As a depressed person in the public, the last thing you would do is shut them in the bedroom and leave them in there for 23 hours a day (CCDHB worker, RP).

This participant reinforces the work of Wortley (2002) and Burrows et al. (2003) who have highlighted the importance of shifts in physical environment as a way of enhancing care-oriented practice. In particular, they emphasise how a ligature-free environment does not have to be entirely void of stimulus and can work to limit the negative impact that isolation has on prisoners, particularly those with mental health issues. Many interviewed staff highlighted how prisoners would benefit from an increase in activities 
and interactions, such as playing cards or reading books, as a way to alleviate boredom and to encourage 'mindfulness' exercises among mentally unwell prisoners. All staff, across both sites, stressed the benefits that a 'step-down' unit would also bring as a way of promoting a more care-oriented approach to 'at-risk' prisoners, who found the isolating nature of the ARU to be a negative experience and who struggled with transitioning from the stark ARU environment to the mainstream prison. The ARU framework New South Wales utilises 'step-down' protocols to ensure that prisoners are subject to a gradual reduction of restrictions, rather than being directly channelled from segregation back into the mainstream prison environment. Such an approach is viewed as allowing prisoners to better 'settle', from one environment to another and is useful for prisoners exhibiting less severe at-risk 'symptoms' (Corrective Services NSW, n.d. a: 8). Similarly, in England and Wales, reformed ARU policy has introduced 'vulnerable prisoners' wings for less extreme cases, bringing successful results in respect of careoriented practice, where low-level 'at-risk' prisoners can be accommodated, observed and supported without experiencing the isolation of segregation units (Mills, 2002).

\section{Prisoner vulnerability and the wider prison environment}

The findings of this thesis, and the varied use of the ARU, indirectly highlight the wider struggles that vulnerable prisoners are forced to cope with as a result of 'everyday prison life' and the stresses or 'pains of imprisonment' (Sykes, 1958: 66). As one worker explained:

People are just trying to survive in prison, actually. And they're doing the best they can. And these are people, the prison population in general, are impoverished in lots of ways - emotionally, intellectually, sometimes physically - they don't have the resources that the normal, average population would have, in dealing with stress... A large proportion of the [prison] community that you're dealing with are really damaged from very early on in their lives and have a very limited repertoire of solutions to how to cope with all of this and a lot of their solutions involve not being able to trust people, staying safe, developing a paranoid way of thinking about the world, because the world isn't a safe place, ever... People just don't know how to live in other ways, actually (CCDHB worker, RP).

Arguably, the notion of being 'at-risk' is synonymous with or perpetuated by the prison experience itself, which is known to have a detrimental impact on many prisoners' wellbeing, mental state and sense of personal worth (Liebling, 2006). Such 
considerations raise broader questions of whether care-oriented practice and the alleviation of emotional distress can ever be achieved within the inherently nontherapeutic penal environment.

As highlighted, successful MDTs can help to downplay security-oriented and risk averse correctional priorities, when responding to 'at-risk' prisoners. Specifically, cohesive, stable and well-resourced MDTs, which operate within progressive and health-centric cultural environments, can help to transform risk factors and 'harmful potentialities' into individualised needs, which can then be addressed with human rather than institutional interests in mind. As such, when developing care-oriented practice in ARUs, a major focus should be on enhancing 'ground-up' rather than 'top-down' initiatives as a way for practice to be improved, which is reliant on strong leadership and support within MDTs. To conclude, this thesis has signposted some practical and immediate possibilities for ARU reform that could be easily implemented or further enhanced to develop a more care-oriented approach to 'at-risk' prisoner management. While such incremental shifts can be highly valuable, the question remains whether small-scale reform to ARUs is a radical enough solution to ensure true care-oriented responses for 'at-risk' prisoners in the future, particularly among those with mental health problems. Moreover, ARUs may be the 'canary in the mine', highlighting gaps and barriers to care-oriented practice across the wider correctional-health framework, hopefully provoking important further research, action and deliberation in the years to come. 


\section{References}

Adams, K. \& Ferrandino, J. (2008). Managing Mentally Ill Inmates in Prisons. Criminal Justice and Behaviour, 35(8), 913-927.

Anasseril, D. (2006). Preventing suicide in prison: a collaborative responsibility of administrative, custodial, and clinical staff. The Journal of the American Academy of Psychiatry and the Law, 34(2), 165-175.

Barker, E., Kolves, K. \& De Leo, D. (2014). Management of Suicidal and Self-harming Behaviors in Prisons: Systemic Literature Review of Evidence-Based Activities. Archives of Suicide Research, 18(3), 227-240.

Baxter, J. (2008). Mãori mental health needs profile: a review of the evidence. Palmerston North: Te Rau Matatini.

Beck, U. (1997). World risk society. London: Polity Press.

Bell, D. (1999). Ethical issues in the prevention of suicide in prison. Australian and New Zealand Journal of Psychiatry, 33, 723-728.

Bersot, H. Y. \& Arrigo, B. A. (2011). The Ethics of Mechanical Restraints in Prisons and Jails: A Preliminary Inquiry from Psychological Jurisprudence. Journal of Forensic Psychology Practice, 11(2-3), 232-264.

Birmingham, L. (2003). The mental health of prisoners. Advances in Psychiatric Treatment, 9(1), 191-201.

Black, J. (2005). The emergence of risk-based regulation and the new public risk management in the United Kingdom. Public Law, Autumn, 512-548.

Borrill, J., Snow, L., Medlicott, D., Teers, R. \& Paton, J. (2005). Learning from 'Near Misses': Interviews with Women who Survived an Incident of Severe Self-Harm in Prison. The Howard Journal of Criminal Justice, 44(1), 57-69.

Brennan, N. (2010, April 12). Prison Staff to the Rescue. Waikato Times. Retrieved from 
http://www.stuff.co.nz/waikato-times/news/3572257/Prison-staff-to-the-rescue.

May 2015.

Brooking, R. (2013, September 22). Torture in New Zealand 1 - the so-called At-Risk cells. Retrieved from http://brookingblog.com/category/medical-torture/. 3 February 2015.

Bullock, K. (2011). The Construction and Interpretation of Risk Management Technologies in Contemporary Probation Practice. British Journal of Criminology, $51,120-135$.

Burrows, T., Brock, A. P., Hulley, S., Smith, C \& Summers, L. (2003). Safer Cells Evaluation: Full Report. London: The Jill Dando Institute of Crime Science.

Camilleri, P., McArthur, M. \& Webb, H. (1999). Suicidal Behaviour in Prisons: A Literature Review. Canberra: Australian Catholic University for ACT Corrections.

Carlton, B. \& Segrave, M. (2014, May 13). Lifting the veil on the crisis in Victoria's prisons. The Conversation. Retrieved from http://theconversation.com/lifting-theveil-on-the-crisis-in-victorias-prisons-25476. 3 November 2014.

Clark, L. (2014). International Expert Explores the Right to Life. Law News, 42. Auckland: Auckland District Law Society.

Cliquennois, G. \& Champetier, B. (2012). A new risk management for prisoners in France: The emergence of a death-avoidance approach. Theoretical Criminology, $17(3), 397-415$.

Clough, P. \& Nutbrown, C. (2007). A Student's Guide to Methodology. London: Sage Publications.

Coid, J., Petruckevitch, A., Bebbington, P., Jenkins, R., Brugha, T., Lewis, G., Farrell, M. \& Singleton, N. (2003). Psychiatric morbidity in prisoners and solitary cellular confinement, II: special ('strip') cells. The Journal of Forensic Psychiatry \& Psychology, 14(2), 320-340. 
Connolly, M. \& Ward, T. (2007). Morals, Rights and Practice in the Human Services: Effective and Fair Decision-Making in Health, Social Care and Criminal Justice. London: Jessica Kingsley Publishers.

Controller and Auditor-General. (2013). Department of Corrections: Managing offenders to reduce reoffending. Wellington: Author.

Corcos, C. D. \& Lewin, R. D. (2001). The use of "safe cells" in the management of suicidal and violent prisoners. Australasian Psychiatry, 9(1), 47-50.

$\begin{array}{lllll}\text { Corrections } & \text { Act, } & \text { No. } & \text { (2004). } & \text { Retrieved from }\end{array}$ http://www.legislation.govt.nz/act/public/2004/0050/latest/DLM294849.html. 3 March 2015.

Corrections Victoria. (n.d.). Deputy Commissioner's Instruction 1.02 - At-risk Procedures. Melbourne: Author.

Corrective Services NSW. (n.d.). Operations Procedures Manual - Section 13.3 Risk Intervention Team Protocol for the Management of Inmates At-risk of Self Harm or Suicide. New South Wales: Author.

Cox, G. H. (1984). Values, Culture and Prison Policy. The Prison Journal, 64(5), 5-15.

Crawley, E. \& Crawley, P. (2008). Understanding Prison Officers: culture, cohesion and conflict. In J. Bennett, B. Crewe \& A. Wahidin (Eds.), Understanding Prison Staff (pp. 134-152). Portland: Willan Publishing.

Crawley, E. (2004). Doing Prison Work: the public and private lives of prison officers. Cullompton: Willan Publishing.

Crawley, E. (2006). Doing Prison Work. In Y. Jewkes \& H. Johnston (Eds.), Prison Readings, Devon: Willan Publishing.

Crewe, B. (2011). Depth, weight, tightness: Revisiting the pains of imprisonment. Punishment \& Society, 13(5), 509-529.

Crewe, B., Warr, J., Bennett, P. \& Smith, A. (2014). The emotional geography of prison life. Theoretical Criminology, 18(1), 56-74. 
Crighton, D. (2002). Suicide in prisons: a critique of UK research. In G. Towl, L. Snow \& M. McHugh (Eds.), Suicide in Prisons. Oxford: Blackwell.

Croucher, S. M. \& Mills, C. (2014). Understanding Communication Research Methods: A Theoretical and Practical Approach. London: Routledge.

Daniel, A. \& Fleming, J. (2006). Suicides in a state corrections system, 1992-2002: A review. Journal of Correctional Health Care, 12, 24-35.

Daniel, A. (2006). Preventing suicide in prison: A collaborative responsibility of administrative, custodial and clinical staff. Journal of the American Academy of Psychiatry and the Law, 34, 165-175.

David, S. \& Codd, H. (2010). Controversial Issues in Prisons. Maidenhead: Open University Press.

Department of Corrections. (2011). Briefing for the Incoming Minister: 2011. Wellington: Author.

Department of Corrections. (2012a). Annual Report 2011/2012. Wellington: Author.

Department of Corrections. (2012b). Corrections Amendment Bill - Department of Corrections Responses to Questions for the Law and Order Committee for Consideration at its Meeting on 29 May 2012. Retrieved from http://www.parliament.nz/resource/ennz/50SCLO_ADV_OODBHOH_BILL11157_1_A242780/fef31f3fac5be94569b3508eb987d 299520d7205. 11 May 2015.

Department of Corrections. (2012c). Prison Service Operations Manual (PSOM). Retrieved from http://www.corrections.govt.nz/resources/Prison-OperationsManual.html. 4 July 2014.

Department of Corrections. (2013a). Annual Report: 1 July 2012 - 30 June 2013. Wellington: Author.

Department of Corrections. (2013b). Media Release: Mental Health Screening Tool Improves Prisoners' Care. Retrieved from 
http://www.corrections.govt.nz/news/media-

releases/2013_media_releases/mental_health_screening_tool_improves_prisoners_ca re.html. 4 June 2015.

Department of Corrections. (2014a). Annual Report: 1 July 2013 - 30 June 2014. Wellington: Author.

Department of Corrections. (2014b, March). High Dependency Unit to expand. Corrections Works. 11-12.

Department of Corrections. (n.d.). Factsheet: Preventing Suicide and Self-Harm Factsheet. Retrieved from http://www.corrections.govt.nz/working_with_offenders/prison_sentences/managing_ offenders/preventing_suicide_and_self_harm.html. 3 February 2015.

Doyle, J. (1997). A trip to Long Bay. Justice Action's Prison Reconnaissance, Framed, 32. Page 20.

Dudley, M., Silove, D. \& Gale, F. (2012). Mental Health and Human Rights: Vision, Praxis and Courage. Oxford: Oxford University Press.

Equality and Human Rights Commission (EHRC). (2012). Human Rights Review 2012: How fair is Britain? An assessment of how well public authorities protect human rights. Retrieved from http://www.equalityhumanrights.com. 19 January 2015.

Ericson, R. (2007). The architecture of risk and power. British Journal of Criminology, $47(6), 955-968$.

Fazel, S. \& Danesh, J. (2002). Serious mental disorder in 23000 prisoners: a systematic review of 62 surverys. Lancet, 359, 545-550.

Feeley, M. \& Simon, J. (1992). The new penology: Notes on the emerging strategy of corrections and its implications. Criminology, 30(4). 171-176.

Gallagher, C. A. \& Dobrin, A. (2005). The association between suicide screening practices and attempts requiring emergency care in juvenile justice facilities. Journal of the American Academy of Child and Adolescent Psychiatry, 44(5), 485-493. 
Garland, D. (2001). The Culture of Control: Crime and Social Order in Contemporary Society. Oxford: Oxford University Press.

Genders, E. \& Player, E. (2014). Rehabilitation, risk management and prisoners' rights. Criminology and Criminal Justice, 14(4), 434-457.

Giacomini, M. (2001). The rocky road: qualitative research as evidence. Evidence Based Medicine, 6(1), 4-6.

Gilbert, N. (1994). Researching social life. London: Sage Publications.

Glaser, W. \& Laster, K. (1990). Are the Mentally Ill Being Criminalised?: Admission of Prisoners to Psychiatric Hospitals Before and After the 1986 Mental Health Act (VIC). Australian \& New Zealand Journal of Criminology, 23(4), 230-240.

Glaser, B. G. \& Strauss, A. L. (2012). The discovery of grounded theory: strategies for qualitative research (7th eds.). New Jersey: Transaction Publishers.

Groves, A. (2004). Blood on the Walls: Self-mutilation in Prisons. Australian \& New Zealand Journal of Criminology, 37(49), 49-64.

Gubrium, J. F., \& Holstein, J. A. (2000). Analyzing interpretive practice. In N. K. Denzin \& Y. S. Lincoln (Eds.), Handbook of qualitative research (pp. 487-508). Thousand Oaks: Sage Publications.

Gurney, J. N. (1985). Not one of the guys: the female researcher in a male-dominated setting. Qualitative Sociology, 8(1), 42-62.

Hannah-Moffat, K. (1999). Moral agent or actuarial subject: Risk and Canadian women's imprisonment. Theoretical Criminology, 3(1), 71-94.

Harvey, D., Hildreth, J., MacLeod, J. \& Moran, P. (2009). Mental Health in the Criminal Justice System: Offender Health and Disabilities. Australasian Journal of Correctional Staff Development, 4(3), 1-18.

Hayes, L. M. (2011). Guide to Developing and Revising Suicide Prevention Protocols within Jails and Prisons. Baltimore: National Centre on Institutions and Alternatives. 
Hennessy, C. (2011, May). Prison Welfare: Making it Impossible to Kill Yourself. Suicide Prevention Information New Zealand Newsletter. Retrieved from: http://www.spinz.org.nz/page/155-newsletter+issues+may-2011. 1 February 2015.

HM Chief Inspector of Prisons. (1990). Suicide and Self-Harm in Prison Service Establishments in England and Wales. London: HMSO.

HM Inspectorate of Prisons (2014). Annual Report 2013-2014. London: Author.

HM Inspectorate of Prisons. (2007). The Mental Health of Prisoners: A Thematic Review of the Care of Prisoners with Mental Health Needs. London: Author.

HM Prison Service \& NHS Executive. (1999). The Future Organisation of Prison Health Care: Report by the Joint Prison Service and National Health Service Executive Working Group. London: Department of Health.

Hood, C., Rothstein, H. \& Baldwin, R. (2004). The Government of Risk: Understanding Risk Regulation Regimes. Oxford: Oxford University Press.

Human Rights Commission. (2009). Comments of the New Zealand Human Rights Commission on New Zealand's Implementation of the United Nations Convention Against Torture. Auckland: Author.

Human Rights Commission. (2012). Monitoring Places of Detention: Annual report of activities under the Optional Protocol of the Convention Against Torture (OPCAT). Auckland: Human Rights Commission.

Independent Police Conduct Authority (IPCA). (2015). Review of Police Custodial Management. Wellington: Author.

Israel, M. \& Hay, I. (2012). Research Ethics in Criminology. In D. Gadd, S. Karstedt \& S. F. Messner (Eds.), The Sage Handbook of Criminological Research Methods (pp. 500516). London: Sage Publications.

Jupp, V. (2001). Triangulation. In E McLaughlin and J Muncie (Eds.). The Sage Dictionary of Criminology. London: Sage Publications. 
Justice Health. (2014). Correctional Suicide Prevention Framework 2014: Working to prevent prisoner and offender suicides in Victorian correctional settings. Melbourne: Department of Justice.

Kauffman, P. (1999). Prisoners. In K. Starmer, European Human Rights Law (pp. 453491). London: Butterworths.

Kelly, D. (2014). Punish or Reform? Predicting Prison Staff Punitiveness. The Howard Journal of Criminal Justice, 53(1), 49-68.

Kemshall, H. (1998). Risk in probation practice. Aldershot: Ashgate.

Kemshall, H. (2003). Understanding Risk in Criminal Justice. Maidenhead: Open University Press.

King, P. (2012). E kore koe e ngaro: Opportunities to improve continuity of primary care for people who are, or have been, imprisoned within the Greater Wellington Region. Wellington: Regional Public Health.

King, R. D. \& Morgan, R. (1980). The Future of the Prison System. Farnborough: Gower Press.

Leach, P. (n.d.). Article 2 ECHR (the right to life) - Positive Obligations. London: London Metropolitan University.

Liebling, A. (1992). Suicides in Prison. London: Routledge.

Liebling, A. (1995). Vulnerability and Prison Suicide. British Journal of Criminology, 35(2), 173-187.

Liebling, A. (2001a). Suicides in prison: Ten years on. Prison Service Journal, 138(7), 3541.

Liebling, A. (2001b). Whose side are we on? Theory, practice, and allegiances in prisons research. British Journal of Criminology, 41(3), 472-484.

Liebling, A. (2002). Suicides in Prison and the Safer Prisons Agenda. Probation Journal, 49(2), 140-150. 
Liebling, A. (2006). The Role of the Prison Environment in Prison Suicide and Prisoner Distress. In G. E. Dear, (Eds.). Preventing Suicide and Other Self-Harm in Prison. London: Palgrave Macmillan.

Liebling, A. (2007). 'Prison Suicide and its Prevention' in Jewkes, Y, (Eds.), Handbook on Prisons. Cullompton: Willan.

Liebling, A. (2014). Postscript: Integrity and Emotion in Prisons Research. Qualitative Inquiry, 20(4), 481-486.

Liebling, A. \& Arnold, A. (2005). Prisons and their Moral Performance: A Study of Values, Quality and Prison Life. Oxford: Oxford University Press.

Liebling, A., Price, D. \& Elliot, C. (1999). Appreciative inquiry and relationships in prison. Punishment and Society, 1(1), 71-98.

Liebling, A., Price, D. \& Shefer, G. (2011). The Prison Officer (2nd ed.). Oxon: Willan Publishing.

Liebling, A., Tait, S., Stiles, A., Durie, L., Harvey, J. \& Rose, G. (2005). An Evaluation of the Safer Locals Programme: Final Report. Cambridge: Cambridge Institute of Criminology (Prisons Research Centre).

Livingstone, P. (2000). Prisoners' rights in the context of the European Convention on Human Rights. Punishment and Society, 4(3), 309-324.

Lyneham, M. \& Chan, A. (2012). Deaths in custody in Australia to 30 June 2011: Twenty years of monitoring by the National Deaths in Custody Program since the Royal Commission into Aboriginal Deaths in Custody. Canberra: Australian Institute of Criminology.

Martynowicz, A. (2011). Oversight of Prison Conditions and Investigations of Deaths in Custody: International Human Rights Standards and Practice in Ireland. The Prison Journal, 91(1), 81-102.

Matfin, C. (2000). Doing research in a prison setting. In V. Jupp, P. Davies \& P. Francis (Eds.), Doing Criminological Research (pp. 215-234). London: Sage Publications. 
Mathiesen, T. (1990). Prison on Trial: A Critical Assessment. Thousand Oaks: Sage Publications.

Maurutto, P. \& Hannah-Moffat, K. (2006). Assembling Risk and the Restructuring of Penal Control. British Journal of Criminology, 46, 428-454.

Medlicott, D. (2001). Narratives of Suicidal Prisoners. London: Ashgate.

Mendez, J. E. (2013). Report of the Special Rapporteur on torture and other cruel, inhuman or degrading treatment or punishment. HRC: United Nations General Assembly. Retrieved from http://www.ohchr.org. 12 January 2015.

Mills, A. (2002). Mental Health In-Reach - the Way Forward for Prison? Probation Journal, 49(2), 107-199.

Mills, A., Meek, R. \& Gojkovic, D. (2012). Partners, guests or competitors: Relationships between criminal justice and third sector staff in prisons. Probation Journal, 59(4), 391-405.

Ministry of Health. (2001). Services for People with Mental Health in the Justice System: Framework for Forensic Mental Health Services. Wellington: Author.

Ministry of Health. (2006). New Zealand Suicide Prevention Strategy: 2006-2016. Wellington: Author.

Morgan, R. D., Steffan, J., Shaw, L. B. \& Wilson, S. (2007). Needs for and Barriers to Correctional Mental Health Services: Inmate Perceptions. Psychiatric Services, 58(9), 1181-1186.

Munro, B. (2012, November 25). People with mental illness falling through the cracks. Otago Daily Times Online News. Retrieved from http://www.odt.co.nz/lifestyle/magazine/236274/people-mental-illness-fallingthrough-cracks. 4 July 2015.

Murphy, T. \& Whitty, N. (2007). Risk and human rights in UK prison governance. British Journal of Criminology, 47(5), 798-816. 
National Health Committee. (2010). Health in Justice: Kia Piki te Ora, Kia Tika! Improving the health of prisoners and their families and whānau: He whakapiki i te ora o ngā mauhere me ō rātou whānau. Wellington: Ministry of Health.

New Zealand Bill of Rights Act, No. 109. (1990). Retrieved from: http://www.legislation.govt.nz/act/public/1990/0109/latest/DLM224792.html. 30 June 2015.

Noaks, L. \& Wincup, E. (2004). Criminological Research: Understanding Qualitative Methods. London: Sage Publications.

O'Malley, P. (2004). The Uncertain Promise of Risk. The Australian and New Zealand Journal of Criminology, 37(3), 323-343.

O’Malley, P. (2010). Crime and Risk. Sydney: Sage Publications.

Office of the Auditor-General. (2008). Performance Audit Report: Mental health service for prisoners. Wellington: Author.

Owers, A. (2006). The Protection of Prisoners' Rights in England and Wales. European Journal of Criminal Policy Research, 12, 85-91.

Pannell, J., Howells, K. \& Day, A. (2003). Prison Officer's Belief's Regarding Self-Harm in Prisoners: An Empirical Investigation. International Journal of Forensic Psychology, 1(1), 103-110.

Patel, M. X, Doku, V. \& Tennakoon, L. (2003). Challenges in recruitment of research participants. Advances in Psychiatric Treatment, 9(3), 229-238.

Pautenaude, A. L. (2004). No Promises, But I'm Willing to Listen and Tell What I Hear. The Prison Journal, (84)69, 69-91.

Prisons and Probation Ombudsman for England and Wales. (2011). Learning from PPO Investigations: Self-inflicted deaths in prison custody, 2007-2009. Retrieved from http://www.ppo.gov.uk/self-inflicted-deaths-in-prison.pdf. 20 January 2015.

Radio New Zealand. (2013, 1 October). Corrections responds to criticism of prison health care.

Retrieved

from 
http://www.radionz.co.nz/national/programmes/morningreport/audio/2571161/corr ections-responds-to-criticism-of-prison-health-care. 5 March 2015.

Radio New Zealand. (2013, 1 October). Government rejects call for review of prison healthcare. Retrieved from http://www.radionz.co.nz/national/programmes/checkpoint/audio/2571235/govtrejects-calls-for-review-of-prison-health-care. 13 December 2014.

Radio New Zealand. (2014, November 28). Initial prison death inquiry 'inadequate'. Retrieved from http://www.radionz.co.nz/news/regional/260463/initial-prisondeath-inquiry-\%27inadequate\%27. Accessed on 3 March 2015.

Rafael, E. J. \& Schutt, R. K. (2012). The Practice of Research in Social Work (3 ${ }^{\text {rd }}$ ed.). London: Sage Publications.

Ramluggun, P. (2013). A critical exploration of the management of self-harm in a male custodial setting: qualitative findings of a comparative analysis of prison staff views on self-harm. Journal of Forensic Nursing, 9(1), 23-24.

Reiter, K. (2014). Making Windows in Walls: Strategies for Prison Research. Qualitative Inquiry, 20(4), 417-428.

Reser, J. (1992). The design of safe and humane police cells: a discussion of some issues relating to Aboriginal people in police custody. In D. Biles \& D. McDonald (Eds.), Deaths in Custody Australia, 1980-1989. Canberra: Institute of Criminology.

Ricciardelli, R., Maier, K. \& Hannah-Moffat, K. (2015). Strategic masculinities: Vulnerabilities, risk and the production of prison masculinities. Theoretical Criminology, 19(1), 1-23.

Robson, B. \& Harris, R. (2007). Hauora: Māori Standards of Health IV. A study of the years 2000-2005. Wellington: Te Ropu Rangahau Hauora e Eru Pomare.

Roguski, M. \& Chauvel, F. (2009). The Effects of Imprisonment on Inmates' and their Families' Health and Wellbeing: prepared for The National Health Committee. Wellington: Litmus. 
Rowan, J. R. (1994). Prevention of suicide in custody. In A. Liebling \& T. Ward (Eds.), Deaths in custody: International perspectives (pp. 166-175). London: Institute for the Study and Treatment of Delinquency.

Sapsford, R. \& Jupp, V. (2006). Data Collection and Analysis (2nd ed.). London: Sage Publications.

Scott, D. \& Codd, H. (2010). Controversial Issues in Prisons. Berkshire: Open University Press.

Scraton, P. (2007). Power, Conflict and Criminalisation. New York: Routledge.

Scraton, P. \& Moore, L. (2005). The Hurt Inside: The Imprisonment of Women and Girls in Northern Ireland. Belfast: Human Rights Commission.

Seddon, T. (2007). Punishment and Madness: Governing Prisoners with Mental Health Problems. Oxon: Routledge.

Seddon, T. (2008). Dangerous liaisons: Personality disorder and the politics of risk. Punishment and Society, 10(3), 301-317.

Sedenu, A. (2005). Safer Custody Group. The British Journal of Forensic Practice, 7(4), 14-20.

Sharkey, L. (2010) 'Does Overcrowding in Prisons Exacerbate the Risk of Suicide among Women Prisoners?' The Howard Journal, 49(2), 111-124.

Sheffer, G. (2012). The impact of performance management culture on prison-based Therapeutic Communities. European Journal of Criminology, 9(4), 407-424.

Short, V., Cooper, J., Shaw, J., Kenning, C., Abel, K. \& Chew-Graham, C. (2009). Custody vs. care: attitudes of prison staff to self-harm in women prisoners - a qualitative study. The Journal of Forensic Psychiatry \& Psychology, 20(3), 408-426.

Sim, J. (1990). Medical power in prisons: the prison medical service in England 1774-1989. Bristol: Open University Press. 
Sim, J. (2002). The future of prison health care: a critical analysis. Critical Social Policy, $22(2), 300-323$.

Sim, J. (2003). Whose Side Are We Not On? Researching Medical Power in Prisons. In S. Tombs \& D. Whyte (Eds.), Unmasking the Crimes of the Powerful. New York: Peter Lang Publishing Incorporated.

Simpson, A. I. F., Brinded, P. M. J., Laidlaw, T. M., Fairley, N. \& Malcolm, F. (1999). The National Study of Psychiatric Morbidity in New Zealand Prisons. Wellington: Department of Corrections.

Skogstad, P., Deane, F. P. \& Spicer, J. (2006). Social Cognitive Determinants of HelpSeeking for Mental Health Problems among Prison Inmates. Criminal Behaviour and Mental Health, 16, 43-59.

Smith, M. (2007). Report on issues involving the criminal justice sector. Wellington: Office of the Ombudsman.

Sparks, R., Bottoms, A. E. \& Hay, W. (1996). Prisons and the Problem of Order. Oxford: Clarendon Press.

Speed, C. (2012). Self-Inflicted Deaths in Prison: An Exploration of INQUEST'S Challenges to State Power. Internet Journal of Criminology. Retrieved from http://www.internetjournalofcriminology.com/Speed_SelfInflicted_Deaths_in_Prison_IJC_Oct_2012.pdf. 2 February 2014.

Stanley, E. (2011). Human Rights and Prisons: A review to the Human Rights Commission. Auckland: Human Rights Commission.

State Services Commission (SSC), the Treasury \& the Department of the Prime Minister and Cabinet (DPMC). (2012). Performance Improvement Framework, Formal Review: Department of Corrections. Wellington: Author.

State Services Commission. (2013, 21 October). High Dependency Unit (HDU), Rimutaka Prison - a first in New Zealand. Retrieved from http://www.ssc.govt.nz/hdu-bpscase-study-oct13. 4 April 2015. 
Sykes, G. (1958). The Society of Captives: A Study of Maximum-Security prison. Princeton: Princeton University Press.

UK Ministry of Justice. (2013). Management of prisoners at-risk of harm to self, to others and from others (safer custody): Prison Service Instruction 2011-64. Retrieved from http://www.justice.gov.uk. 1 October 2014.

UN General Assembly. (1966). International Covenant on Civil and Political Rights (ICCPR). United Nations Treaty Series, 99(171). Retrieved from: http://www.refworld.org/docid/3ae6b3aa0.html. 3 March 2015.

Urquhart, C. (2013). Grounded theory for qualitative research: a practical guide. London: Sage Publications.

Victorian Ombudsman. (2014). Investigation into deaths and harm in custody (Ombudsman Act 1973). Melbourne: Author.

Wacquant, L. (2002). The curious eclipse of prison ethnography. Anthropology News, $50(1), 4-5$.

Wakem, B. \& McGee, M. (2012). Investigation of the Department of Corrections in relation to the Provision, Access and Availability of Prisoner Health Services. Wellington: Office of the Ombudsman.

Walklate, S. \& Mythen, G. (2011). Beyond risk theory: Experiential knowledge and 'knowing otherwise'. Criminology \& Criminal Justice, 11(2), 99-113.

Whitty, N. (2011). Human rights as risk: UK prisons and the management of risk and rights. Punishment \& Society, 13(2), 123-148.

Williams, T. A. (1983). Custody and Conflict: An Organizational Study off Prison Officers' Roles and Attitudes. Australian \& New Zealand Journal of Criminology, 16(1), 44-55.

Willis, J. W. (2007). Foundations of Qualitative Research: Interpretive and Critical Approaches. Thousand Oaks: Sage Publications.

World Health Organisation (WHO). (2007). Preventing suicide in jails and prisons. Geneva: WHO Press. 
Wortley, E. (2002). Situational Prison Control: Crime Prevention in Correctional Institutions. Cambridge: Cambridge University Press.

\section{List of cases}

Keenan v UK [2001] 33 EHRR 913.

Toia v Department of Corrections [2014] NZHC 867 


\section{Appendices}

Appendix A - Copy of research agreement between myself and NZDoC

Appendix B - Copy of Human Ethics Approval

Appendix C - Participant Information Form

Appendix D - Participant Consent Form

Appendix E - Participant Recruitment Flyer

Appendix F - Interview Schedule

Appendix G - Number of incidences of self-harm in New Zealand prisons, 2005-2015

Appendix H - Number of deaths in custody in New Zealand prisons, 2000-2015 
Appendix A: Research Agreement 


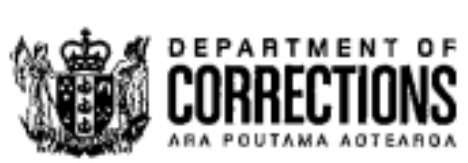

Research Agreement

Between

\section{Parties}

Her Majesty the Queen acting by and through the Chief Executive of the Department of Corrections (the Department)

And

Alexis Harris and Victoria University of Wellington (jointly the Researcher), each of whom is jointly and severally liable with the other in terms of their obligations.

\section{Background}

A. The Researcher has submitted to the Department an "Application to Undertake Research", set out as Schedule One (Application).

B. The Department has accepted and approved the Application.

C. This Agreement documents the terms and conditions upon which the Department allows the Researcher to conduct research within the Department's facilities and with prisoners, offenders managed in the community and / or Departmental clients and / or staff (collectively, participants).

\section{Terms and conditions}

The Department grants permission to the Researcher to undertake this research on the following terms and conditions:

1 This research is entitled "The Role of Multi-Agency Collaboration in the Development of Therapeutic Models of Care for 'At-Risk' Male Prisoners in New Zealand" and will be undertaken in accordance with this Agreement, as well as the methods and detail outlined in the Application, at all times.

2 The Researcher confirms that the research has been endorsed by a University Head of Department or supervisor, or manager of a reputable research-related organisation.

3 The Researcher confirms that the Researcher has approval from an accredited institutional ethics committee, or the proposal has been reviewed by a recognised human ethics body.

4 Where applicable, the Researcher will obtain informed consent (as that term is defined in the Application) from all participants, keep a record of that consent, and provide the Department with evidence of that consent if requested by the Department. 
5 The Researcher will undertake the research liaising Carolyn O'Fallon, of the Department of Corrections (Department's Representative) or their replacement person.

6 Only those individuals identified in the Application and named in this Agreement as the Researcher may undertake the research. Prior to commencing the research, the Researcher will meet all of the Department's security and other requirements relating to access to any institution and to participants.

7 If the Researcher wishes, at any stage, for additional individuals to undertake research (or to substitute individuals) it must first obtain the Department's consent in writing and it acknowledges that those additional individuals will first need to clear appropriate security and additional checks before undertaking research.

8 Any information which could lead to participants' identification will not appear in any form (verbal or written information) in the Document, any publication, teaching or presentations. This clause 10 survives expiry or termination of the research project and this Agreement.

9 The Researcher knows and understands that permission to undertake the research is conditional upon compliance with the requirements of the Privacy Act 1993. In particular the Researcher accepts their obligation under that act to ensure that:

- the information gained will be used solely for purposes directly linked to the research project, and will not be published in a form that could reasonably be expected to identify any individual [Principles 10(f)(i), 10(f)(ii), 11(h)(i), and 11(h)(ii)]; and

- no other person, other than a person assisting the Researcher with the analysis or research, will have access to participants' personal information. In particular, the Researcher will ensure that the information is protected by such security safeguards as is reasonable under the circumstances to take, against loss [Principle 5(a)(i)]; access, modification or disclosure [Principle 5(a)(ii)].

10 The Researcher will not make any copy or remove any Departmental records consulted in the course of the research that identifies offenders or participants, without the written permission of the Department.

11 During the course of the research, the Researcher will at all times be sensitive to the operational environment in which the research is undertaken. This includes awareness of the staffing and resource constraints that the Department operates within.

12 The Researcher will take all possible steps to protect the participants from discomfort, distress or embarrassment. Participants' welfare and dignity will take precedence over the requirements of the research at all times.

13 Where information is disclosed to the Researcher that signifies actual and immediate risks to the safety and welfare of participants or other persons, these concerns shall be brought to the attention of the relevant Unit or Service Manager in the first instance, as well as the Department's Representative.

14 Other than information being gathered for the research and other than information disclosed as per clause 10 above, the Researcher agrees to keep confidential all information about the Department and its operations about which 
the Researcher becomes aware. This clause 14 survives expiry or termination of the research project and this Agreement.

15(a) The Researcher must provide a copy of the final draft of the thesis, paper, research report or similarly described document, the production of which represents the purpose of and summarises the research (collectively, Document) to the Department's Representative for review prior to the document being finalised. At least six weeks should be allowed for this review.

15(b) The Researcher understands and agrees that:

- The Department may require alterations to the content of the Document to correct factual inaccuracies, and the researcher agrees to make any such alterations;

- The Department may request that the Researcher remove confidential information, as per clause 10 .

- The Department may ask for other content changes that it considers appropriate.

- The Department may require the Document to carry a disclaimer stating that the Document does not represent the views of the Department. The Researcher agrees to comply with any request made by the Department in this regard.

Once the Document has had alterations made to it and carries a disclaimer (if required) the Researcher will resubmit the altered Document for further review.

16 The process set out in clause 15 will be repeated if necessary until the Department's Representative advises the Researcher in writing that the Department is satisfied with the Document (at which point it will become the "final agreed Document"). No further changes to the final agreed Document will be permitted without a repeat of the process in this clause 16 and clause 15.

17 Prior to the time at which the Document becomes the final agreed Document the Researcher will not publish or otherwise disclose, orally or in writing, to any third party, or use in any way for any purpose, including educational or further research purposes, any information obtained through the Department during the term of this agreement, without the written approval of the Department. This clause 17 includes any interim reports or findings of the research that the Researcher intends to release or publish, prior to producing the final agreed Document.

18 Following acceptance of the final agreed Document under clauses 15 and 16 ,the Researcher is required to provide the Department with copies of any presentation, draft paper for publication, or other representation of the research findings, prior to the material being made available to the intended audience.

19 The Department will not unreasonably withhold or delay its decision-making under clauses 15, 16 and 17.

20 The Researcher must provide the Department with an electronic copy of the final agreed Document. If the final agreed Document is to be published in hard copy, on the request of the Department, the Researcher will, at its cost, provide it with one copy of the Document.

21 The Department will treat any breach by the Researcher of this Agreement very seriously. In the event of a breach, the Department may: 
- terminate this Agreement immediately by written notice; or

- give written notice of a breach and of a reasonable time that the breach must be remedied; and

- terminate this Agreement if the breach is not remedied within that time; or

- suspend the continuation of this Agreement and therefore the right of the Researcher to access Department premises, participants or other resources; and

- in the event of any breach not consider any future applications by the Researcher to undertake research.

These remedies are listed as examples only and are entirely without prejudice to any other remedies the Department may have for breach, at law or otherwise.

\section{Signed as an Agreement:}

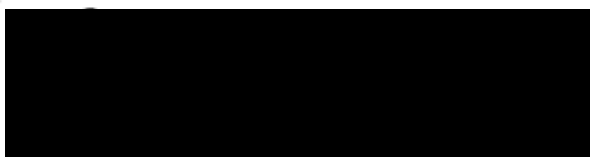

Name: ALEXIS HARRIS

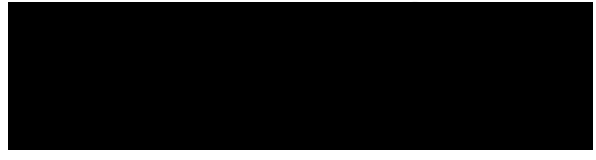

Name:

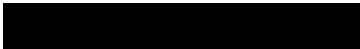

Designation: General Manager, Service Development $24 / 7 / 114$.

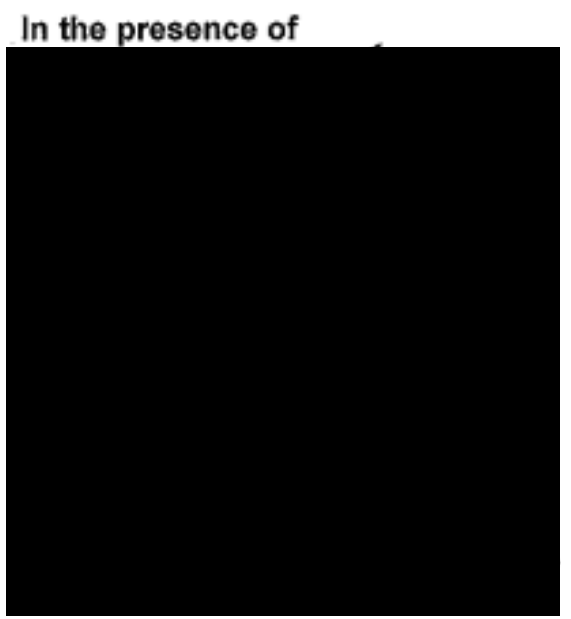

In the presence of

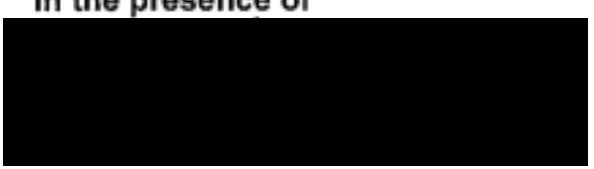

Name:

Occupation: Personal Assistant

Address: $\quad$ Private Box 1206

Wellington 6140 
Appendix B: Human Ethics Approval 
MEMORANDUM

\begin{tabular}{l|l}
\hline TO & Alexis Harris \\
\hline COPY TO & Elizabeth Stanley \\
\hline FROM & Dr Allison Kirkman, Convener, Human Ethics Committee \\
\hline
\end{tabular}

\begin{tabular}{l|l}
\hline DATE & 13 August 2014 \\
\hline PAGES & 1 \\
\hline
\end{tabular}

\begin{tabular}{l|l}
\hline SUBJECT & $\begin{array}{l}\text { Ethics Approval: } 21213 \\
\text { The Role of Multi-Agency Collaboration in the Development of } \\
\text { Therapeutic Models of Care for 'At-Risk' Male Prisoners in New } \\
\text { Zealand }\end{array}$ \\
\hline
\end{tabular}

Thank you for your application for ethical approval, which has now been considered by the Standing Committee of the Human Ethics Committee.

Your application has been approved from the above date and this approval continues until 1 November 2015. If your data collection is not completed by this date you should apply to the Human Ethics Committee for an extension to this approval.

Best wishes with the research.

Allison Kirkman

Human Ethics Committee 
Appendix C: Participant Information Form (RP) 


\section{VICTORIA UNIVERSITY OF WELLINGTON \\ Te Whare Wananga o te Upoko o te Ika a Maui

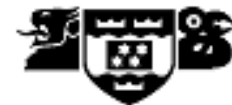

\section{At-Risk Unit Research Project}

My name is Alexis Harris and I am a post-graduate student at Victoria University of Wellington (VUW). I am undertaking research that focuses on multi-agency collaborations and therapeutic care in At-Risk Units in men's prisons in New Zealand. I am looking to conduct a number of 1.5 hour interviews with a number of prison officers and nurses working within this Unit.

\section{About the Research}

It is hoped that this research will be helpful to policy and practice development, as well as being academically valuable, by:

- Providing an overview of how other At-Risk Units are operating and developing in a number of correctional facilities overseas;

- Identifying strengths, weaknesses and possible improvements that could be made to the current At-Risk system in New Zealand prisons;

- Examining how the development of multi-disciplinary collaborations are perceived and experienced by those working within them, as a means of enhancing therapeutic approaches to prisoner care/management.

The outcomes of this research will be submitted to VUW in the form of a thesis, to fulfill the requirements for a Master of Arts in Criminology. The outcomes of this research may be used in academic publications (e.g. journal articles or conference papers).

\section{About the Interview}

If you agree to take part in this research, the Principal Corrections Officer (PCO) will organise an interview time that is suitable to you. It is likely that interviews will take place within the prison, during work hours. I am hoping to conduct interviews within this facility's Unit from the $17^{\text {th }}-21^{\text {st }}$ November 2014.

During the interview, you will be asked to clarify how things currently operate on the ground, drawing from personal experiences. You will also be asked about your thoughts and experiences in relation to: how multi-disciplinary collaborations are perceived and experienced by you and your colleagues; and, how collaborative working practices can enhance therapeutic approaches towards 'at risk' prisoners.

Your responses will be digitally recorded and I may also take some written notes on your comments. Digital recordings will be transcribed following the interview. It is thought that the interview will take a maximum of 1.5 hours. 


\section{Consent}

Your participation is completely voluntary. If you do agree to take part, you will be asked to sign a consent form. Please be aware that:

- You do not have to answer any question that you do not want to;

- You may withdraw your consent at any time during or up to one month after the interview. You do not need to disclose a reason for wanting to withdraw from the study. If you do withdraw, all your responses will be completely removed from my data collection.

\section{Feedback}

Following the interview, you can check the written transcript and suggest changes or additions to the researcher. You can also request a summary of the research to be sent to you at the end of the project.

\section{Confidentiality}

If you agree to take part in this research:

- All of your responses, unless you note otherwise, will be strictly confidential to the researcher;

- Your name will not appear on any paperwork or digital recording that contains your interview data. Details of your name and contact details will be stored in a separate place from your interview data;

- All information will be held in a secure place. Access to the interview data will be limited to the researcher and her supervisor;

- All digital recordings and recorded personal information will be destroyed one year after the final thesis has been academically submitted.

\section{Anonymity}

Unless agreed otherwise, your name will not be included in any publication or document relating to this work. Your responses will be cited in any outputs under a relevant generic title such as 'Prison Officer' or 'Nurse'.

\section{Contacting the Researcher}

If you are interested in taking part in this research, please contact the Principal Corrections Officer to arrange an interview time.

For further information on this project, you can contact me directly at:
Alexis Harris
Institute of Criminology,
Victoria University of Wellington
PO Box 600 , Wellington 6140
Email: alexis.harris@vuw.ac.nz
Telephone: 04-463 8444 
Appendix D: Participant Consent Form 


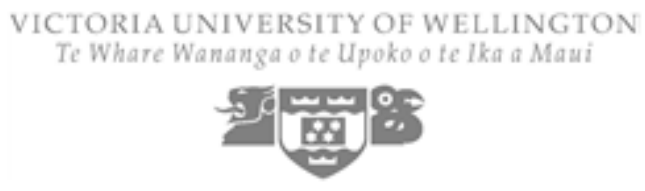

Participant Consent Form

The research has been fully explained to me and I have had the opportunity to ask any questions I have about it.

I agree to the interview being recorded and noted.

I know that I can check a transcript of the interview when it is completed.

I may withdraw my consent at any time during the interview and up to one month after the interview without the need to disclose any reason for doing so. If this happens, any data I have provided will be deleted.

I note that any information I provide will be kept confidential and, unless I agree otherwise, reported in a non-identifiable form.

I understand that the information I have provided will not be used for any other purpose or released to others without my written consent.

I am aware that following final submission of the researcher's thesis, all information collected will be destroyed.

I, [print name] __ have read this form and voluntarily consent to participate in this research.

Signature Date

\section{Feedback}

Please tick the boxes below and provide a mail address if you would like further feedback.

Yes, I would like to check my interview transcript

Yes, I would like to receive a summary of this research

Mailing address:

\section{Anonymity Exclusion}

Tick here if you consent to your name being used within publications 
Appendix E: Participant Recruitment Flyer (RP)

\section{VICTORIA UNIVERSITY OF WELLINGTON \\ Te Whare Wananga o te Upoko o te Ika a Maui \\ 59 \\ Are you interested in taking part in an upcoming research project?}

\section{Inform thinking on working in an At-Risk Unit}
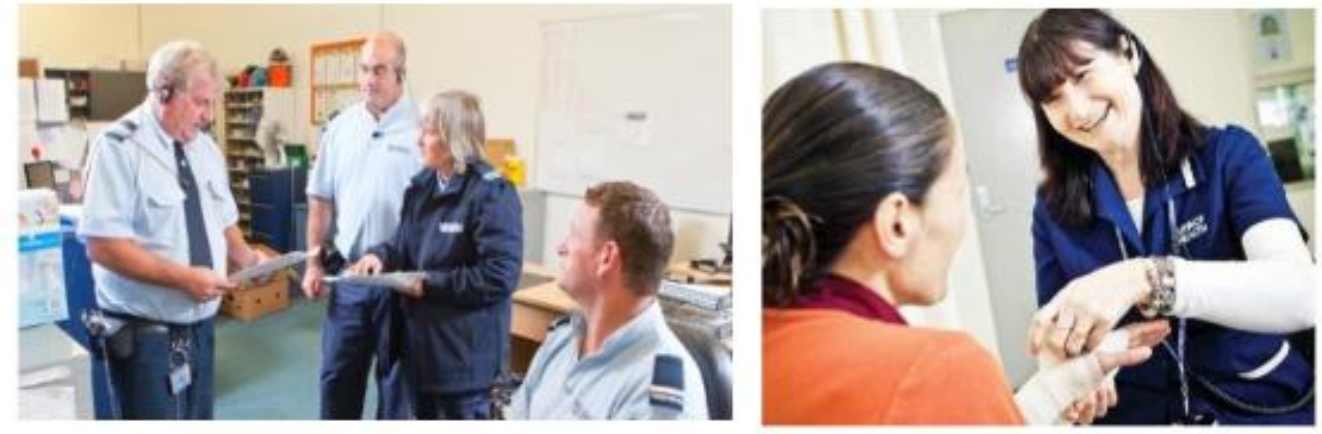

I am undertaking research that focuses on multi-agency collaborations and therapeutic care in At-Risk Units in men's prisons in New Zealand. I am looking to conduct a number of 1.5 hour interviews with prison officers and nurses working within this Unit.

If you agree to take part in this research, an interview time that is suitable to you will be organised. It is likely that interviews will take place within the prison, during work hours. I am hoping to conduct interviews within this facility's Unit from the 17th-21stNovember 2014.

If you are interested in taking part in this research, or for further information, please contact your Principal Corrections Officer directly to arrange an interview time.

Alternatively, for further information on this research project, you can contact me directly: Email: alexis.harris@vuw.ac.nz

Telephone: 04463 8444

\begin{tabular}{|c|c|c|c|c|c|c|c|c|c|c|c|c|c|c|c|c|c|c|c|c|c|}
\hline 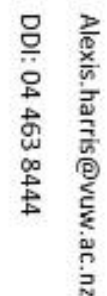 & 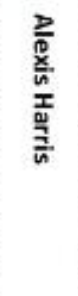 & 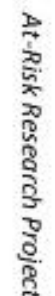 & 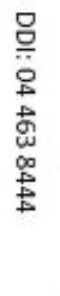 & 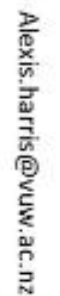 & $\begin{array}{l}\frac{2}{0} \\
\frac{x}{4} \\
\frac{x}{0} \\
\frac{1}{3} \\
\frac{1}{4}\end{array}$ & 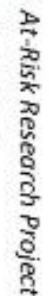 & 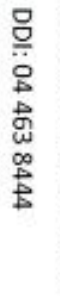 & 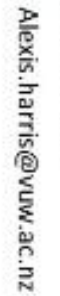 & 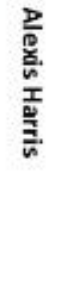 & 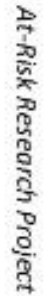 & 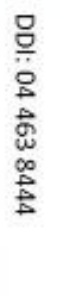 & 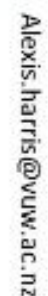 & 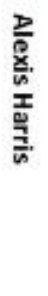 & 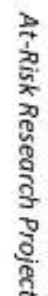 & 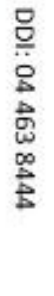 & 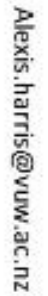 & 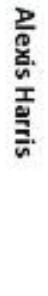 & 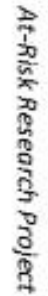 & 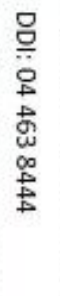 & 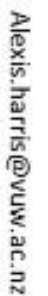 & $\begin{array}{l}\frac{2}{0} \\
\frac{x}{u} \\
\frac{1}{0} \\
\frac{0}{n} \\
\frac{1}{4}\end{array}$ \\
\hline
\end{tabular}


Appendix F: Interview Schedule 


\section{Interview schedule - At-Risk research project}

Introduction and informed consent

- Introduce myself and the purposes of the research project

- Talk through consent form and information sheet, ensure consent is informed and obtained

- Provide an opportunity for any questions to be raised with the interviewer

Port I:

Background and experience

- Can you please tell me a bit about your 'journey' - how you came to work in your role/discipline and how you came to work in the ARU this prison?

- How much work experience have you had in other prisons?

- How much experience have you had working within ARUs?

Clarification of policy and proctice

- Can you describe a 'normal day' in the At-Risk Unit - is there is such a thing?

- What are the most pressing issues or challenges that you face in the every-day working environment of the ARU?

- In your view, what are the main aims and purposes of ARUs?

- How does this Unit compare to working in the rest of the prison estate? (Key differences in policy? Types of prisoners? Relationships with prisoners? Staffing?)

- From your experience, would you say that most prisoners in the ARU present with suicidal or self-harming ideations? Have you seen prisoners placed in ARUs for reasons other than selfharm/suicide risk? If yes, how do you manage the diversity of needs (diverse population)? (Examples)

- Has your perspective on 'risk' changed as a result of working in this Unit? (Examples)

- Regarding prisoners' departure from ARU: who makes this decision? On what basis?

- To what extent do matters of security/staff safety impact on this decision-making process?

Multi-disciplinary working relotionships

- From an outsider's perspective, it seems like there would be competing interests among staff with different disciplinary backgrounds - how does this play out in practice?

- Can you describe to me the usual staff structure and presence in the ARU? E.g. how many nurses in an ARU most days, frequency of contact with external agencies/individuals, rollover of staff.

- What are the benefits of multi-disciplinary teams? And, what are the difficulties?

- Can you explain to me the current decision-making process, in terms of determining a prisoner's care in the ARU?

- Have your working practices changed as a consequence of working with others from different professional backgrounds / being exposed to diverse professional approaches? If yes, how? (examples)

- Do you think the prison would benefit from an increase in multi-disciplinary teams? If yes, what do MDTs add to your work? Would MDTs be reserved for special units, or general approach? 
A theropeutic environment

- What does 'therapeutic care' or 'therapy' mean to you in the context of the ARU? Do you consider ARUs to be a therapeutic environment? Why/why not?

- In your view, what would need to happen in order for ARUs to be transformed into a more therapeutic regime?

- One of the big debates in the ARU literature is about the tension between 'care' and 'control'. Where do you think the line falls within this ARU? Examples?

- Drawing from your experiences and perceptions, would there be merit in making the Units more therapeutic? What would this mean in practice? What about making prisons more therapeutic? 
Part II:

International best practice

\section{Overarching strategic/principled framework}

In Australia, Victorio has developed a strategic framework to manage and respond to at-risk prisoners. This framework is underpinned by foundational principles that ensure consistency of practice and culture across all prisons in Victoria. Examples of such principles include: 'suicide should be seen as preventable' and 'activities should be promoted as having a therapeutic and not punitive intention'. Other international frameworks (NSW, UK) emphasise the importance of humane, tailored responses to at-risk prisoners; for example, they repeatedly note that segregation should only be a matter of last resort and recognise that each prisoner has a diverse ronge of needs.

- Do you think NZ should develop a similar overarching strategic framework?

aYes aNo

- If no, why not?

- If yes, what would it need to contain? And, how might it promote greater levels of therapeutic care for at-risk prisoners?

- What difficulties might there be in implementing this kind of framework?

2. Wider utilisation of services/activities/external interventions for at-risk prisoners

In the UK and in some parts of Austrolia, at-risk prisoners are able to take part in games (such as XX) or be oided by prisoner support schemes, such as progrommes run by the Samoritans. In New South Woles, policy stipulates that counselling services should be offered to any prisoners who show signs of distress.

- Do you think wider use of similar initiatives would promote greater levels of therapeutic care for at-risk prisoners in New Zealand?

aYes aNo

- If no, why not?

- If yes, what services, activities, external interventions would be useful?

- How do you think this would impact on the current practice and operation of ARUs in New Zealand?

\section{A graduated/tiered response model}

All prisoners in Victoria are subject to a risk assessment by a mental health professional at their initial reception into prison. Once assessed, prisoners are categorised within a graduated response model (e.g. $S 1$ - immediate risk; 53 - potential risk). Similarly, NSW has a 'step down' protocol for staff to utilise when a prisoners' risk level decreases. This ensures that restrictions on prisoners are gradually reduced, rather than being directly channelled from segregation back into the mainstream prison environment. 
- Do you think a graduated risk model, such as the ones mentioned above, would promote greater levels of therapeutic care for at-risk prisoners in New Zealand?

$\square$ Yes $\quad$ No

- If no, why not?

- If yes, what benefits would this graduated system bring?

- What difficulties might there be in implementing such a model?

4. Structure of multi-agency interactions

- In the UK (?), policy focuses on the joint management and expertise of healthcore and custodial staff, and emphasises consensus decision-making. Policy olso emphasises the importance of 'continuity in staff ottendonce', allowing the same stoff members to be involved with o respective prisoner's care, wherever possible.

a. Do you think greater levels of joint management between healthcare and custodial staff, based on consensus decision-making, would promote greater levels of therapeutic care for at-risk prisoners New Zealand?

aYes $\quad$ No

b. Do you think greater continuity of staff attendance within ARUs would promote greater levels of therapeutic care for at-risk prisoners New Zealand?
a Yes
$\square$ No

With regard to both questions:

- How would these approaches change current ways of working?

- In implementing them, what benefits would there be?

- What difficulties might emerge in pursuing these approaches?

\section{Further Questions}

- Which of the above international practices, if any, would you consider to be most useful?

- If you had all the power and a blank cheque, would you want to change ARUs or the policy surrounding them in any other way? What would you do?

Closing

- Touch on the key points that have been raised during the interview and ask the participant if there is anything they would like to add or if there are any further comments they wish to make. 
SELF HARM THREAT TO LIFE INCIDENTS*

\begin{tabular}{|c|c|c|c|c|c|c|c|c|c|c|}
\hline Prison & $2005-06$ & $2006-07$ & $2007-08$ & $2008-09$ & $2009-10$ & $2010-11$ & $2011-12$ & $2012-13$ & 2013-14 & $\begin{array}{l}2014-15 \text { to } 31 \\
\text { March } 2015\end{array}$ \\
\hline ACRP & 1 & & 3 & 4 & 1 & 3 & & & & \\
\hline AROHATA PRISON & & 1 & 1 & 2 & & & 1 & & & \\
\hline ARWCF & & 1 & 1 & & 1 & 1 & & & 2 & \\
\hline AUCKLAND PRISON & 7 & 1 & 3 & 2 & 2 & 4 & & & 5 & 1 \\
\hline CHRISTCHURCH PRISON & 6 & 6 & 5 & 3 & 5 & & 1 & & 2 & \\
\hline $\begin{array}{l}\text { CHRISTCHURCH } \\
\text { WOMENS }\end{array}$ & 4 & 6 & 1 & & 4 & 3 & & 1 & & \\
\hline HAWKES BAY PRISON & 1 & & & 1 & 6 & 1 & 2 & 2 & 2 & \\
\hline INVERCARGILL PRISON & & & 1 & & & & & & & \\
\hline MANAWATU PRISON & 3 & 2 & 2 & 4 & & 1 & & & & \\
\hline MECF & & & & & & & 1 & 3 & 1 & \\
\hline MT EDEN MENS & 1 & 3 & 4 & 2 & 3 & & & & & \\
\hline MT EDEN WOMENS & 3 & & & & & & & & & \\
\hline NEW PLYMOUTH & 1 & & 1 & 2 & 1 & 2 & & & & \\
\hline NRCF & 1 & & & & & & & & & \\
\hline OCF & & & 2 & 1 & 1 & 1 & 1 & & 1 & \\
\hline RIMUTAKA PRISON & & 1 & 2 & 4 & 4 & & & & 1 & \\
\hline ROLLESTON PRISON & 1 & & & 1 & & & & & & \\
\hline SHCF & & & & 2 & & 1 & & & 1 & \\
\hline TONGARIRO/RANGIPO & 1 & & & 1 & & & & & & \\
\hline WAIKERIA PRISON & 8 & 2 & 5 & 1 & 1 & 3 & & & 4 & \\
\hline WELLINGTON PRISON & 1 & & & & & & & & & \\
\hline WHANGANUI PRISON & 3 & 3 & & 3 & 2 & 3 & & 1 & & 1 \\
\hline Grand Total & 42 & 26 & 31 & 33 & 31 & 23 & 6 & 7 & 19 & 2 \\
\hline
\end{tabular}

*Intentional act of harm to oneself which would most probably have led to death if there was no immediate intervention (includes all attempted suicides)

Appendix G: Number of incidences of self-harm in New Zealand prisons, 2005-201535

35 Bowman, J. (Personal communication, 20 April, 2015). 
Appendix H: Number of deaths in custody in New Zealand prisons, 2000-201536

\begin{tabular}{|c|c|c|c|c|}
\hline \multicolumn{5}{|c|}{ Inspectorate Investigations - Death in Custody } \\
\hline Reporting Year & $\begin{array}{c}\text { Natural } \\
\text { Causes }\end{array}$ & Suicide & Homicide & TOTAL \\
\hline $2000-2001$ & 5 & 6 & 1 & 12 \\
\hline $2001-2002$ & 7 & 7 & 0 & 14 \\
\hline $2002-2003$ & 4 & 4 & 1 & 9 \\
\hline $2003-2004$ & 6 & 9 & 1 & 16 \\
\hline $2004-2005$ & 6 & 7 & 1 & 14 \\
\hline $2005-2006$ & 5 & 6 & 1 & 12 \\
\hline $2006-2007$ & 5 & 5 & 1 & 11 \\
\hline $2007-2008$ & 6 & 4 & 0 & 10 \\
\hline $2008-2009$ & 12 & 4 & 1 & 17 \\
\hline $2009-2010$ & 6 & 9 & 0 & 15 \\
\hline $2010-2011$ & 13 & 10 & 0 & 23 \\
\hline $2011-2012$ & 9 & 5 & 0 & 14 \\
\hline $2012-2013$ & 8 & 3 & 0 & 11 \\
\hline $2013-2014$ & 10 & 3 & 0 & 13 \\
\hline $2014-2015$ & 7 & 7 & 1 & 15 \\
\hline TOTAL & 109 & 89 & 8 & 206 \\
\hline
\end{tabular}

Updated 31 March 2015

Note: The reporting year is 01 July to 30 June

36 Bowman, J. (Personal communication, 20 April, 2015). 